\title{
Classification of torus manifolds with codimension one extended actions
}

\section{Shintaro KUROKI}

\begin{tabular}{|c|l|}
\hline Citation & OCAMI Preprint Series \\
\hline Issue Date & 2009 \\
\hline Type & Preprint \\
\hline Textversion & Author \\
\hline Rights & For personal use only. No other uses without permission. \\
\hline Relation & $\begin{array}{l}\text { This is a pre-print of an article published in Transformation } \\
\text { Groups. The final authenticated version is available online at: } \\
\text { https://doi.org/10.1007/s00031-011-9136-7 }\end{array}$ \\
\hline
\end{tabular}

From: Osaka City University Advanced Mathematical Institute http://www.sci.osaka-cu.ac.jp/OCAMI/publication/preprint/preprint.html 


\title{
Classification of torus manifolds with codimension one extended actions
}

\author{
Shintarô KUROKI
}

\begin{abstract}
The goal of this paper is to classify torus manifolds $\left(M^{2 n}, T^{n}\right)$ with codimension one extended $G$-actions $\left(M^{2 n}, G\right)$ up to essential isomorphism, where $G$ is a compact, connected Lie group whose maximal torus is $T^{n}$. For technical reasons, in this paper, we do not assume that torus manifolds are omnioriented. As a result, we have the following two results: (1) if there is no exceptional orbit in extended $G$-actions, there are five kinds of $\left(M^{2 n}, G\right)$ 's (Theorem 1.1, 1.2); (2) otherwise, there are two kinds of $\left(M^{2 n}, G\right)$ 's (Theorem 1.3). As a corollary of these results, we also have that if $M^{2 n}$ is a non-singular toric variety or a quasitoric manifold with codimension one extended $G$-actions, then $M^{2 n}$ is a complex projective bundle over a product of complex projective spaces.
\end{abstract}

\section{Introduction}

This paper is a continuation of $[\mathbf{1 2}]$ and [13] devoted to the study of the extended $G$-actions on torus manifolds $\left(M^{2 n}, T^{n}\right)$, where a torus manifold is an even dimensional oriented manifold $M^{2 n}$ acted on by a half-dimensional torus $T^{n}$ with non-empty fixed point set, and $G$ is a compact, connected Lie group whose maximal torus is $T^{n}$. In the first paper [12], we classified the homogeneous (unoriented) torus manifolds and their transformation groups up to essential isomorphism, where here an unoriented torus manifold means a torus manifold which is not assumed omniorientations. By using classical Lie theory, we proved such torus manifolds are only products of even dimensional spheres and complex projective spaces divided by finite groups. In the second paper [13], we classified quasitoric manifolds with codimension 1 extended $G$-actions up to essential isomorphism and studied relations with moment-angle manifolds. In order to classify such quasitoric manifolds, we classified more general class which involve them, i.e., simply connected torus manifolds with codimension 1 extended $G$-actions whose two singular orbits are also simply connected torus manifolds. To classify such torus manifolds, we used the part of the Uchida's method in [17]. The Uchida's method is the strong method to classify codimension 1 compact Lie group actions up to essential isomorphism. In the case that we apply the Uchida's method to classify codimension 1 actions,

2000 Mathematics Subject Classification. 57S25.

Key words and phrases. Cohomogeneity one action, Non-singular toric variety, Quasitoric manifold, Toric topology, Torus manifold.

The author was supported in part by Fudan University and the Fujyukai foundation. 
we usually need to divide our proof into two cases (compare the method of [11, Section 7,8$]$ and that of $[\mathbf{1 1}$, Section 10, 11]). In particular, for torus manifolds with codimension 1 extended $G$-actions, (as we mentioned in [13]) we divide our proof into the following two cases: the case that two singular orbits of $G$-actions are torus manifolds; and the case that one of two singular orbits of $G$-actions is not a torus manifold. Remark that if a singular orbit is a torus manifold then this is a homogeneous torus manifold (see Lemma 2.1); therefore, we know such singular orbit type by using [12] (see Lemma 2.2). In the previous paper [13], we only studied the former case because of its purpose. However, in general, the latter case also occurs (see [13, Example 3.5]). The goal of this paper is to classify all unoriented torus manifolds with codimension one extended $G$-actions up to essential isomorphism. In particular, in this paper, we put a special emphasis on the proof of the latter case, because the proof of the former case is almost similar to that of [13] even if in the case of the classification of such unoriented torus manifolds.

Now we state the main result of this paper. Put $m_{j}, l_{i} \in \mathbb{N} \cup\{0\}$ for $j=$ $1, \cdots, b$ and $i=1, \cdots, a$, and $\mathcal{A} \subset \prod_{j=1}^{b} \mathbb{Z}_{2}$. We get the following three theorems (see Section 3.1, 5.1 and 8.1 for detail).

TheOREM 1.1 (Theorem 3.1). Suppose a torus manifold $M$ has a codimension one extended $G$-action. If there are two singular orbits and both of them are torus manifolds, then $(M, G)$ is essentially isomorphic to

$$
\left(\prod_{j=1}^{b} S^{2 m_{j}} \times_{\mathcal{A}} N, \quad \prod_{j=1}^{b} S O\left(2 m_{j}+1\right) \times H\right),
$$

such that $(N, H)$ is one of the following three types:

\begin{tabular}{|c|c|}
\hline$N$ & $H$ \\
\hline \hline$\left(\prod_{i=1}^{a} S^{2 l_{i}+1}\right) \times_{T^{a}} S\left(\mathbb{C}_{\mathfrak{a}}^{k} \oplus \mathbb{R}\right)$ & $\prod_{i=1}^{a} S U\left(l_{i}+1\right) \times U(k)$ \\
\hline$\left(\prod_{i=1}^{a-1} S^{2 l_{i}+1}\right) \times_{T^{a-1}} P\left(\mathbb{C}_{\mathfrak{b}}^{k_{1}} \oplus \mathbb{C}^{k_{2}}\right)$ & $\prod_{i=1}^{a-1} S U\left(l_{i}+1\right) \times S\left(U\left(k_{1}\right) \times U\left(k_{2}\right)\right)$ \\
\hline$\prod_{i=1}^{a} \mathbb{C} P\left(l_{i}\right) \times S\left(\mathbb{R}^{2 k} \oplus \mathbb{R}\right)$ & $\prod_{i=1}^{a} S U\left(l_{i}+1\right) \times S O(2 k)$ \\
\hline
\end{tabular}

where $k, k_{1}, k_{2} \in \mathbb{N}$.

THEOREM 1.2 (Theorem 8.1). Suppose a torus manifold $M$ has a codimension one extended $G$-action. If there are two singular orbits and one of them is not a torus manifold, then $(M, G)$ is essentially isomorphic to

$$
\left(\prod_{j=1}^{b-1} S^{2 m_{j}} \times_{\mathcal{A}} N, \quad \prod_{j=1}^{b-1} S O\left(2 m_{j}+1\right) \times H\right),
$$

such that $(N, H)$ is one of the following two types:

\begin{tabular}{|c|c|}
\hline$N$ & $H$ \\
\hline \hline$\prod_{i=1}^{a} S^{2 l_{i}+1} \times{ }_{T^{a}} S\left(\mathbb{C}_{\mathfrak{c}}^{k_{1}} \oplus \mathbb{R}^{2 k_{2}-1}\right)$ & $\prod_{i=1}^{a} S U\left(l_{i}+1\right) \times U\left(k_{1}\right) \times S O\left(2 k_{2}-1\right)$ \\
\hline$\prod_{i=1}^{a} \mathbb{C} P\left(l_{i}\right) \times S\left(\mathbb{R}^{2 k_{1}} \oplus \mathbb{R}^{2 k_{2}-1}\right)$ & $\prod_{i=1}^{a} S U\left(l_{i}+1\right) \times S O\left(2 k_{1}\right) \times S O\left(2 k_{2}-1\right)$ \\
\hline
\end{tabular}

where $k_{1} \in \mathbb{N}, k_{2} \geq 2$.

TheOREM 1.3 (Theorem 5.1). Suppose a torus manifold $M$ has a codimension one extended $G$-action. If there is an exceptional orbit, then $(M, G)$ is essentially 
isomorphic to

$$
\left(\prod_{j=1}^{b} S^{2 m_{j}} \times_{\mathcal{A} \times \mathbb{Z}_{2}} N, \quad \prod_{j=1}^{b} S O\left(2 m_{j}+1\right) \times H\right),
$$

such that $(N, H)$ is one of the following two types:

\begin{tabular}{|c|c|}
\hline$N$ & $H$ \\
\hline \hline$\left(\prod_{i=1}^{a} S^{2 l_{i}+1}\right) \times_{T^{a}} S\left(\mathbb{C}_{\mathfrak{c}}^{k_{1}} \oplus \mathbb{R}\right)$ & $\prod_{i=1}^{a} S U\left(l_{i}+1\right) \times U\left(k_{1}\right)$ \\
\hline$\prod_{i=1}^{a} \mathbb{C} P\left(l_{i}\right) \times S\left(\mathbb{R}^{2 k_{1}} \oplus \mathbb{R}\right)$ & $\prod_{i=1}^{a} S U\left(l_{i}+1\right) \times S O\left(2 k_{1}\right)$ \\
\hline
\end{tabular}

where $k_{1} \in \mathbb{N}$.

From the above theorems (see Section 3.1, 5.1 and 8.1 for detail), the finite group $\mathcal{A}$ or $\mathcal{A} \times \mathbb{Z}_{2}$ acts only on $\prod_{j=1}^{b} S^{2 m_{j}}$ and on the fibre of $N$ (not on $\prod_{i=1}^{a} S^{2 l_{i}+1}$ and $\left.\prod_{i=1}^{a} \mathbb{C} P\left(l_{i}\right)\right)$. Therefore, we also have that if an unoriented torus manifold has a codimension one extended $G$-action, then this manifold is a fibre bundle over the homogeneous torus manifold $\prod_{j=1}^{b} S^{2 m_{j}} / \mathcal{A}^{\prime} \times \prod_{i=1}^{a} \mathbb{C} P\left(l_{i}\right)$ (see $[\mathbf{1 2}]$ ) whose fibre is a complex projective space $\mathbb{C} P(l)$, an even dimensional sphere $S^{2 m}$, or an even dimensional real projective space $\mathbb{R} P(2 m)$. Therefore, we can easily show the following corollary:

COROLlary 1.4. If a non-singular toric variety or a quasitoric manifold $M$ has a codimension one extended $G$-action, then $(M, G)$ is essentially isomorphic to

$$
\left(\prod_{i=1}^{a-1} S^{2 l_{i}+1} \times_{T^{a-1}} P\left(\mathbb{C}_{\mathfrak{b}}^{k_{1}} \oplus \mathbb{C}^{k_{2}}\right), \quad \prod_{i=1}^{a-1} S U\left(l_{i}+1\right) \times S\left(U\left(k_{1}\right) \times U\left(k_{2}\right)\right)\right) .
$$

Remark that the manifold in Corollary 1.4 is equivariantly diffeomorphic to

$$
\prod_{i=1}^{a-1} \mathbb{C}_{o}^{l_{i}+1} \times{ }_{\left(\mathbb{C}^{*}\right)^{a-1}} P\left(\mathbb{C}_{\mathfrak{b}}^{k_{1}} \oplus \mathbb{C}^{k_{2}}\right)
$$

where $\mathbb{C}_{o}^{l_{i}+1}=\mathbb{C}^{l_{i}+1}-\{o\}$ and $\mathbb{C}^{*}=\mathbb{C}-\{0\}$ (removed the origin).

The organization of this paper is as follows. In Section 2, we first set up some notation and basic facts from $[\mathbf{1 2}, \mathbf{1 3}]$. Then we know that, in order to classify codimension one extended actions, we need to consider the three cases: the cases (1), (2) and (3). In Section 3, we classify the case (1), i.e., two singular orbits are torus manifolds. The proof of this case is similar to the previous classification in [13]. Hence, we can apply the arguments of the proofs in [13], and show Theorem 1.1. In Section 4, we give a preparation for the cases (2) and (3). In particular, we know the following key fact in this section: in order to classify the cases (2) and (3), we may only study the isotropy subgroup $K_{2} \subset G$ and its slice representation $\sigma_{2}$ by Remark 4.2 , where $G / K_{1}$ is the singular orbit of the codimension 1 extended action which is a torus manifold and $G / K_{2}$ is the other non-principal orbit. In Section 5, we state the main theorem and give the remark for the case (2), i.e., one of non-principal orbits is an exceptional orbit. In particular, we divide this case into (2)-(a) and (2)-(b). In Section 6 and 7, we study the cases (2)-(a) and (2)-(b) respectively, and prove Theorem 1.3. In Section 8, we state the main theorem of the case (3), i.e., one of the non-principal orbits is not a torus manifold but a singular orbit, and we divide this case into the cases (3)-(a) and (3)-(b). In Section 9 and 10 , we classify the case (3)-(a) and the case (3)-(b) respectively, and prove Theorem 1.2 . 


\section{Review of the previous papers}

In order to classify codimension 1 extended actions, in this section, we recall the basic facts (see $[\mathbf{1}, \mathbf{8}, \mathbf{1 2}, \mathbf{1 3}, \mathbf{1 7}]$ for detail).

2.1. Definition of torus manifold. We start with recalling the definition of the torus manifold. Let $\left(M^{2 n}, T^{n}\right)$ be a pair $2 n$-dimensional, compact, connected manifold $M^{2 n}$ and a half dimensional torus $T^{n}$. We call $\left(M^{2 n}, T^{n}\right)$ a torus manifold if it satisfies that

(1) $T^{n}$-action on $M^{2 n}$ is amost effective, i.e., the intersection of all isotropy subgroups is a finite subgroup in $T^{n}$;

(2) its fixed point set is non-empty, i.e., $M^{T} \neq \emptyset$.

A torus manifold $\left(M^{2 n}, T^{n}\right)$ is often denoted by $(M, T)$ or $M$ simply.

In the paper [8], the definition of torus manifolds involves the choice of orientations of manifold $M$ and its characteristic submanifolds called omniorientation on $M$. Because we will classify extended actions up to essential isomorphism in this paper, we do not need to choice an omniorientation on $M$. Moreover, the $T$-action on $M$ does not need to be effective (also see [12, Remark 2.1]). We also call such torus manifold an unoriented torus manifold in this paper.

Note that $M^{T}$ is finite for unoriented torus manifolds $(M, T)$ as well as the torus manifolds in the sense of $[\mathbf{8}]$.

2.2. Codimension 1 extended $G$-actions and their singular orbits. Let $(M, T)$ be a torus manifold, and let $G$ be a compact, connected Lie group whose maximal torus is $T$. We next recall the basic facts for extended $G$-actions of $(M, T)$.

Suppose a $T^{n}$-action on $M^{2 n}$ extends to a $G$-action on $M$ with codimension 1 principal orbits, i.e., $(2 n-1)$-dimensional orbit. Then we call $(M, T)$ has a codimension 1 extended $G$-action, and such extended $G$-action on $M$ is denoted by $(M, G)$. We will classify such $(M, G)$ up to essential isomorphism, where here we say that $(M, G)$ is essentially isomorphic to $\left(M^{\prime}, G^{\prime}\right)$ if these induced effective actions are weak equivariantly diffeomorphic (see $[\mathbf{1 2}, \mathbf{1 3}]$ for detail)

For codimension 1 extended actions of $(M, T)$, the following lemma holds (see [13, Lemma 3.1, 3.2]).

Lemma 2.1. Suppose that an (unoriented) torus manifold $\left(M^{2 n}, T^{n}\right)$ has a codimension 1 extended $G$-action. Then a $G$-orbit $G / K_{1}$ of $T$-fixed points is a singular orbit in $(M, G)$, i.e., $\operatorname{dim} G / K_{1}<2 n-1$.

Furthermore, there is some subtorus $T^{\prime} \subset T$ such that $\left(G / K_{1}, T^{\prime}\right)$ is an (unoriented) torus manifold.

Proof. In the previous paper $[\mathbf{1 3}]$, we assume the orientation of the torus manifold. However, we can also apply the proofs of [13, Lemma 3.1, 3.2] to the case of unoriented torus manifolds. Hence, we can prove this lemma with a method similar to the proofs of [13, Lemma 3.1, 3.2].

In this paper, we do not need to consider the orientation on the singular orbit $G / K_{1}$. Hence, we can directly apply the main result in [12] to a homogeneous torus manifold $G / K_{1}$. Moreover, we get the following lemma using the argument in $[\mathbf{1 3}$, Section 3.3]. 
LEMMA 2.2. Suppose that a torus manifold $\left(M^{2 n}, T^{n}\right)$ extends to a codimension 1 extended action. Then this codimension 1 extended action is essentially isomorphic to $(M, G)$ which satisfies that, for its singular orbit $G / K_{1}$ with $M^{T} \cap G / K_{1} \neq \emptyset$, the pair $\left(G, K_{1}\right)$ is as follows:

$$
\left(\prod_{i=1}^{a} S U\left(l_{i}+1\right) \times \prod_{j=1}^{b} S O\left(2 m_{j}+1\right) \times G_{1}^{\prime \prime}, \prod_{i=1}^{a} S\left(U\left(l_{i}\right) \times U(1)\right) \times \mathcal{S}_{1} \times G_{1}^{\prime \prime}\right)
$$

where $\prod_{j=1}^{b} S O\left(2 m_{j}\right) \subset \mathcal{S}_{1} \subset \prod_{j=1}^{b} S\left(O\left(2 m_{j}\right) \times O(1)\right)$ and $G_{1}^{\prime \prime}$ is a product of connected, simple Lie groups and tori.

2.3. Slice representations of $G / K_{1}$. In this subsection, we study the slice representation of $G / K_{1}$ for the case of unoriented torus manifolds.

Due to Lemma $2.1, G / K_{1}$ is a torus manifold. Therefore, we can put $\operatorname{dim} G / K_{1}=$ $2 n-2 k_{1}$ for $k_{1} \geq 1$. Let $K_{1}^{\prime}=\prod_{i=1}^{a} S\left(U\left(l_{i}\right) \times U(1)\right) \times \mathcal{S}_{1}$ (see Lemma 2.2), and let $Z(X)$ be the centralizer of a subgroup $X$ in $O\left(2 k_{1}\right)$, i.e., $Z(X)=\left\{g \in O\left(2 k_{1}\right) \mid g x=\right.$ $x g$ for all $x \in X\}$.

Using a method similar to the proof of [13, Lemma 5.6], we have the following lemma for $K_{1}=K_{1}^{\prime} \times G_{1}^{\prime \prime}$ and the slice representation $\sigma_{1}: K_{1} \rightarrow O\left(2 k_{1}\right)$.

Lemma 2.3. For $G_{1}^{\prime \prime}$ in $K_{1}=K_{1}^{\prime} \times G_{1}^{\prime \prime}$, there are the following two cases:

$$
G_{1}^{\prime \prime}=S U\left(k_{1}\right) \times T^{1}, \text { and } G_{1}^{\prime \prime}=S O\left(2 k_{1}\right) .
$$

For these two cases, the slice representation $\sigma_{1}: K_{1}=K_{1}^{\prime} \times G_{1}^{\prime \prime} \rightarrow O\left(2 k_{1}\right)$ satisfies the following list:

\begin{tabular}{|c|c|c|c|}
\hline & $G_{1}^{\prime \prime}$ & $\sigma_{1}\left(G_{1}^{\prime \prime}\right)$ & $\sigma_{1}\left(K_{1}^{\prime}\right)$ \\
\hline \hline$(1)$ & $S U\left(k_{1}\right) \times T^{1}$ & $U\left(k_{1}\right) \subset O\left(2 k_{1}\right)$ & $Z\left(U\left(k_{1}\right)\right) \simeq T^{1} \subset U\left(k_{1}\right) \subset O\left(2 k_{1}\right)$ \\
\hline (2) & $S O\left(2 k_{1}\right)$ & $S O\left(2 k_{1}\right)$ & $Z\left(S O\left(2 k_{1}\right)\right)=\left\{ \pm I_{2 k_{1}}\right\} \subset O\left(2 k_{1}\right)$ \\
\hline
\end{tabular}

where the right list means $\sigma_{1}\left(K_{1}^{\prime}\right) \subset Z\left(U\left(k_{1}\right)\right)$ for $(1)$ and $\sigma_{1}\left(K_{1}^{\prime}\right) \subset Z\left(S O\left(2 k_{1}\right)\right)$ for (2).

Furthermore, the image of $\sigma_{1}$ is in $S O\left(2 k_{1}\right)$, i.e., $\sigma_{1}: K_{1} \rightarrow S O\left(2 k_{1}\right)$.

REMARK 2.4. We give the following four remarks.

(1) If $G_{1}^{\prime \prime}=S O\left(2 k_{1}\right)$ then we can regard $k_{1} \geq 2$, because $S O(2) \simeq T^{1}=$ $S U(1) \times T^{1}$ for $k_{1}=1$.

(2) In the previous paper $[\mathbf{1 3}], \sigma_{1}\left(K_{1}^{\prime}\right)$ is always connected, because $K_{1}$ is connected. However, in this paper, $K_{1}$ is not always connected (because $G_{1}^{\prime \prime}$ is connected but $K_{1}^{\prime}$ might not be connected for $\left.K_{1}=K_{1}^{\prime} \times G_{1}^{\prime \prime}\right)$.

(3) Moreover, in [13], we can assume $m_{j} \geq 2$ (where $m_{j}$ is defined in Lemma $2.2)$. However, in this paper, we assume $m_{j} \geq 1$.

(4) $K_{1} / K \cong K_{1}^{o} / K^{o} \cong S^{2 k_{1}-1}$ because $\operatorname{dim} G / K_{1}=2 n-2 k_{1}$ and the tubular neighborhood of $G / K_{1}$ is $G \times_{K_{1}} D^{2 k_{1}}$ such that $K_{1}$ acts transitively on $\partial D^{2 k_{1}}\left(\cong K_{1} / K\right)$ through $\sigma_{1}$.

In order to apply the same arguments in $[\mathbf{1 3}]$ to the case $m_{j} \geq 1$, we need to study the case $m_{j}=1$. Let $r_{j}: \prod_{j=1}^{b} S O\left(2 m_{j}+1\right) \rightarrow S O\left(2 m_{j}+1\right)$ be the natural projection to the $j$-th factor. Note that we have for $\mathcal{S}_{1}$ in Lemma 2.2

$$
r_{j}\left(\mathcal{S}_{1}\right)=S O\left(2 m_{j}\right) \quad \text { or } \quad S\left(O\left(2 m_{j}\right) \times O(1)\right) \subset S O\left(2 m_{j}+1\right) .
$$

The following lemma holds. 
Lemma 2.5. Assume $m_{j}=1$. Then there are the following two cases:

(1) if $r_{j}\left(\mathcal{S}_{1}\right)=S O\left(2 m_{j}\right)(=S O(2))$, then we can regard $S O\left(2 m_{j}+1\right)=S O(3)$ as $S U(2)=S U\left(l_{a+1}+1\right)$ up to essential isomorphism;

(2) if $r_{j}\left(\mathcal{S}_{1}\right)=S\left(O\left(2 m_{j}\right) \times O(1)\right)(=S(O(2) \times O(1)))$, then there is some inclusion $\iota: S(O(2) \times O(1)) \rightarrow \mathcal{S}_{1}$ such that $\operatorname{Im} \iota \cap S O\left(2 m_{j}\right)=S O\left(2 m_{j}\right)$, and for the slice representation $\sigma_{1}$ we have

$$
\sigma_{1} \circ \iota(S(O(2) \times O(1))) \subset\left\{ \pm I_{2 k_{1}}\right\} \subset S O\left(2 k_{1}\right) .
$$

Proof. The first statement can be easily proved using the fact that $S O(3) \approx$ $S U(2)$, i.e., these Lie algebras are same (also see [13, Section 2.2]). We may only prove the second statement.

Suppose $r_{j}\left(\mathcal{S}_{1}\right)=S\left(O\left(2 m_{j}\right) \times O(1)\right)(=S(O(2) \times O(1)))$. Because $\prod_{j=1}^{b} S O\left(2 m_{j}\right) \subset$ $\mathcal{S}_{1} \subset \prod_{j=1}^{b} S\left(O\left(2 m_{j}\right) \times O(1)\right)$, we can easily see that there is some inclusion $\iota: S(O(2) \times O(1)) \rightarrow \mathcal{S}_{1}$ such that $\operatorname{Im} \iota \cap S O\left(2 m_{j}\right)=S O\left(2 m_{j}\right)$. We may only prove that this inclusion $\iota$ satisfies $\sigma_{1} \circ \iota(S(O(2) \times O(1))) \subset\left\{ \pm I_{2 k_{1}}\right\} \subset S O\left(2 k_{1}\right)$. Let

$$
J=\left(\begin{array}{ll}
0 & 1 \\
1 & 0
\end{array}\right) \in O(2)
$$

Then $J^{2}=I_{2}$. Therefore, by the right list in Lemma 2.3, we have that

$$
\sigma_{1} \circ \iota\left(\left(\begin{array}{cc}
J & 0 \\
0 & -1
\end{array}\right)\right) \in\left\{ \pm I_{2 k_{1}}\right\} \subset U\left(2 k_{1}\right) \subset S O\left(2 k_{1}\right) \text {. }
$$

If $G_{1}^{\prime \prime}=S O\left(2 k_{1}\right)$, then the statement $\sigma_{1} \circ \iota(S(O(2) \times O(1))) \subset\left\{ \pm I_{2 k_{1}}\right\}$ is straightforward because of Lemma 2.3. Assume $G_{1}^{\prime \prime}=S U\left(k_{1}\right) \times T^{1}$. Now we can put

$$
\sigma_{1} \circ \iota\left(\left(\begin{array}{cc}
A & 0 \\
0 & 1
\end{array}\right)\right)=A^{\gamma} \in S O(2) \subset S O\left(2 k_{1}\right)
$$

for $A \in S O(2)$, where $\gamma \in \mathbb{Z}$ and $A^{\gamma} \in S O(2) \simeq T^{1} \subset U\left(k_{1}\right) \subset S O\left(2 k_{1}\right)$ for the diagonal subgroup $T^{1} \subset U\left(k_{1}\right)$, because $S O(2)$ is the abelian group. Hence, by Eq. (2.1), we have that

$$
\begin{aligned}
A^{\gamma} & =\sigma_{1} \circ \iota\left(\left(\begin{array}{cc}
A & 0 \\
0 & 1
\end{array}\right)\right) \\
& =\sigma_{1} \circ \iota\left(\left(\begin{array}{cc}
J & 0 \\
0 & -1
\end{array}\right)\left(\begin{array}{cc}
A & 0 \\
0 & 1
\end{array}\right)\left(\begin{array}{cc}
J & 0 \\
0 & -1
\end{array}\right)\right) \\
& =\sigma_{1} \circ \iota\left(\left(\begin{array}{cc}
A^{-1} & 0 \\
0 & 1
\end{array}\right)\right) \\
& =A^{-\gamma} .
\end{aligned}
$$

It follows that $\gamma=0$; hence, we have the second statement.

If $m_{j} \geq 2$, then we can easily show that $\sigma_{1} \circ \iota \circ r_{j}\left(\mathcal{S}_{1}\right) \subset\left\{ \pm I_{2 k_{1}}\right\}$. Hence, by the above Lemma 2.5, we can regard $\sigma_{1}\left(\mathcal{S}_{1}\right) \subset\left\{ \pm I_{2 k_{1}}\right\}$. This corresponds with the previous fact that $\sigma_{s}\left(\prod_{j=1}^{b} S O\left(2 m_{j}\right)\right)=\{e\}$ in [13, Lemma 7.1, 7.2, 7.3]. Because $\left\{ \pm I_{2 k_{1}}\right\}$ is the center of $S O\left(2 k_{1}\right)$, we can use the same argument in the previous paper [13, Section 7] for analyzing the slice representation $\sigma_{1}$. 
2.4. Global structures of codimension 1 extended actions. We next study the global structure of $(M, G)$. Even if $M$ is non-oriented, the following structure theorem holds (see [1, 8.2 Theorem in Chapter IV]):

THEOREM 2.6. Let $(M, G)$ be a $G$-manifold $M$ with codimension 1 orbits. If every orbit is principal, then $M$ is a $G / K$-bundle over $S^{1}$. Otherwise, there are two non-principal orbits $G / K_{1}$ and $G / K_{2}$ such that $K_{1} \cap K_{2} \supset K$ (K is the principal isotropy subgroup). Furthermore, there exists a closed, invariant tubular neighborhood $X_{s}$ of $G / K_{s}$ for $s=1,2$ such that

$$
M=X_{1} \cup X_{2} \quad \text { and } \quad X_{1} \cap X_{2}=\partial X_{1}=\partial X_{2}=G / K,
$$

where $\partial X_{s}$ means the boundary of $X_{s}$.

Because of Lemma 2.1, we can assume $G / K_{1}$ is a singular orbit and a torus manifold throughout this paper; moreover, because of Theorem 2.6, there are the following three cases:

(1) $G / K_{2}$ is a torus manifold (automatically $G / K_{2}$ is a singular orbit);

(2) $G / K_{2}$ is an exceptional orbit, namely, $\operatorname{dim} G / K_{2}=\operatorname{dim} G / K=2 n-1$;

(3) $G / K_{2}$ is not a torus manifold but a singular orbit.

We call the above cases the case (1), (2) and (3), respectively. Remark that if $M$ is simply connected, then we do not need to consider the case $(2)$ (see $[\mathbf{1 3}$, Theorem 2.6] or [17, Lemma 1.2.1]). From the next section, we start to classify for each above case.

Before we go to the next section, we introduce the following Lemma 2.7 for the attaching map of $f: \partial X_{1} \rightarrow \partial X_{2}$. In the final part of the classification, we compute the attaching maps $f$ from $\partial X_{1}$ to $\partial X_{2}$, and construct the manifold $M(f)=X_{1} \cup_{f} X_{2}$ by using $f$. For two attaching maps $f$ and $f^{\prime}$, we know whether $M(f)$ and $M\left(f^{\prime}\right)$ are diffeomorphic or not, by making use of the following Uchida's criterion (see [17, Lemma 5.3.1]).

Lemma 2.7 (Uchida's criterion). Let $f, f^{\prime}: \partial X_{1} \rightarrow \partial X_{2}$ be G-equivariant diffeomorphisms. Then $M(f)$ is equivariantly diffeomorphic to $M\left(f^{\prime}\right)$ as $G$-manifolds, if one of the following conditions are satisfied:

(1) $f$ is G-diffeotopic to $f^{\prime}$;

(2) $f^{-1} f^{\prime}$ is extendable to a G-equivariant diffeomorphism on $X_{1}$;

(3) $f^{\prime} f^{-1}$ is extendable to a G-equivariant diffeomorphism on $X_{2}$.

We remark that this criterion also holds for non-orientable cases.

Using the above criterion (1), we can take the attaching map $f$ from the group $N(K ; G) / N(K ; G)^{o}$, where $N(K ; G)$ is the normalizer of $K$ in $G$ and $N(K ; G)^{o}$ is its identity component (also see [13, Section 8.1])).

\section{The case (1): two singular orbits are torus manifolds}

The goal of this section is to classify the case (1). So, in this section, we assume that the other singular orbit $G / K_{2}$ is also a torus manifold. Then we can put $\operatorname{dim} G / K_{s}=2 n-2 k_{s}$ for $k_{s} \geq 1$ and $s=1,2$. Note that the proof of this case is similarly to that of the previous classification [13]. 
3.1. Notations and main theorem. First, we state the main theorem of this section. In order to state it, we prepare some notations. A manifold $X \times_{H} Y$ denotes a quotient manifold of $X \times Y$ divided by a free $H$-action. The manifold $\left(\prod_{i=1}^{a} S^{2 l_{i}+1}\right) \times_{T^{a}} S\left(\mathbb{C}_{\mathfrak{a}}^{k} \oplus \mathbb{R}\right)$ is the quotient manifold of $\left(\prod_{i=1}^{a} S^{2 l_{i}+1}\right) \times S\left(\mathbb{C}_{\mathfrak{a}}^{k} \oplus \mathbb{R}\right)$ divided by the following $T^{a}$-action: $T^{a}$ acts on $\prod_{i=1}^{a} S^{2 l_{i}+1}$ by the $a$-times product of the scaler $S^{1}$-action on $S^{2 l_{i}+1} \subset \mathbb{C}^{l_{i}+1}$ for $i=1, \cdots, a$ (in other words, $T^{a}$ acts on $\prod_{i=1}^{a} S^{2 l_{i}+1}$ naturally); and $T^{a}$ acts on the $2 k$-dimensional sphere $S\left(\mathbb{C}_{\mathfrak{a}}^{k} \oplus\right.$ $\mathbb{R}) \subset \mathbb{C}_{\mathfrak{a}}^{k} \oplus \mathbb{R}$ through the representation $\mathfrak{a}: T^{a} \rightarrow S^{1}$ such that $\mathfrak{a}\left(t_{1}, \cdots, t_{a}\right) \mapsto$ $t_{1}^{\alpha_{1}} \cdots t_{a}^{\alpha_{a}}$ for some $\alpha_{1}, \cdots, \alpha_{a} \in \mathbb{Z}$, that is, $\mathbb{C}_{\mathfrak{a}}^{k} \simeq \mathbb{C}^{k}$ (as a vector space) is the representation space of the representation $\mathfrak{a}\left(S^{1}\right.$ acts on this space by the scaler multiplication). The manifold $\left(\prod_{i=1}^{a-1} S^{2 l_{i}+1}\right) \times_{T^{a-1}} P\left(\mathbb{C}_{\mathfrak{b}}^{k_{1}} \oplus \mathbb{C}^{k_{2}}\right)$ is the projectify of the complex vector bundle $\left(\prod_{i=1}^{a-1} S^{2 l_{i}+1}\right) \times_{T^{a-1}}\left(\mathbb{C}_{\mathfrak{b}}^{k_{1}} \oplus \mathbb{C}^{k_{2}}\right)$, where $T^{a-1}$ acts on $\prod_{i=1}^{a-1} S^{2 l_{i}+1}$ naturally; and $T^{a-1}$ acts on the representation space $\mathbb{C}_{\mathfrak{b}}^{k_{1}} \simeq \mathbb{C}^{k_{1}}$ through the representation $\mathfrak{b}: T^{a-1} \rightarrow S^{1}$, and on $\mathbb{C}^{k_{2}}$ trivially. A group $\mathcal{A}$ is a subgroup of $\prod_{j=1}^{b} \mathbb{Z}_{2}$, where $\mathbb{Z}_{2}$ is generated by the antipodal involution on $S^{2 m_{j}}$ for $j=1, \cdots, b$.

Now we may state the main theorem in this section.

THEOREM 3.1. Suppose a torus manifold $M$ has a codimension one extended G-action. If there are two singular orbits and both of them are torus manifolds, then $(M, G)$ is essentially isomorphic to

$$
\left(\prod_{j=1}^{b} S^{2 m_{j}} \times_{\mathcal{A}} N, \quad \prod_{j=1}^{b} S O\left(2 m_{j}+1\right) \times H\right)
$$

such that $(N, H)$ is one of the followings:

\begin{tabular}{|c|c|c|}
\hline & $N$ & $H$ \\
\hline \hline$(a)$ & $\left(\prod_{i=1}^{a} S^{2 l_{i}+1}\right) \times_{T^{a}} S\left(\mathbb{C}_{\mathfrak{a}}^{k} \oplus \mathbb{R}\right)$ & $\prod_{i=1}^{a} S U\left(l_{i}+1\right) \times U(k)$ \\
\hline$(b)$ & $\left(\prod_{i=1}^{a-1} S^{2 l_{i}+1}\right) \times_{T^{a-1}} P\left(\mathbb{C}_{\mathfrak{b}}^{k_{1}} \oplus \mathbb{C}^{k_{2}}\right)$ & $\prod_{i=1}^{a-1} S U\left(l_{i}+1\right) \times S\left(U\left(k_{1}\right) \times U\left(k_{2}\right)\right)$ \\
\hline$(c)$ & $\prod_{i=1}^{a} \mathbb{C} P\left(l_{i}\right) \times S\left(\mathbb{R}^{2 k} \oplus \mathbb{R}\right)$ & $\prod_{i=1}^{a} S U\left(l_{i}+1\right) \times S O(2 k)$ \\
\hline
\end{tabular}

where $\mathcal{A}$ acts on $\prod_{j=1}^{b} S^{2 m_{j}}$ as the subgroup $\prod_{j=1}^{b} \mathbb{Z}_{2}$ and on the fibre of $N$ through the following representations:

(a): $\sigma_{\mathbb{C}}: \mathcal{A} \rightarrow\{ \pm 1\} \subset S^{1}$ on $S\left(\mathbb{C}_{\mathfrak{a}}^{k} \oplus \mathbb{R}\right) \cap \mathbb{C}_{\mathfrak{a}}^{k} ;$

(b): $\sigma_{\mathbb{C}}: \mathcal{A} \rightarrow\{ \pm 1\} \subset S^{1}$ on $\mathbb{C}_{\mathfrak{b}}^{k_{1}}$-factor in $P\left(\mathbb{C}_{\mathfrak{b}}^{k_{1}} \oplus \mathbb{C}^{k_{2}}\right)$;

(c): $\sigma_{\mathbb{R}}: \mathcal{A} \rightarrow\left\{ \pm I_{2 k}\right\} \subset S O(2 k)$ on $S\left(\mathbb{R}^{2 k} \oplus \mathbb{R}\right) \cap \mathbb{R}^{2 k}$;

respectively.

Here, $G$-actions on $M$ are as follows: $\prod S O\left(2 m_{j}+1\right)$ and $\prod S U\left(l_{i}+1\right)$ act naturally on $\prod S^{2 m_{j}}$ and $\prod S^{2 l_{i}+1}$, respectively; and $U(k), S\left(U\left(k_{1}\right) \times U\left(k_{2}\right)\right)$ and $S O(2 k)$ act naturally on $\mathbb{C}_{\mathfrak{a}}^{k}, \mathbb{C}_{\mathfrak{b}}^{k_{1}} \oplus \mathbb{C}^{k_{2}}$ and $\mathbb{R}^{2 k}$, respectively.

From the next subsection, we start to prove the above theorem.

3.2. Singular isotropy subgroups and images of their slice representations. Because $G / K_{2}$ is a torus manifold, the pair $\left(G, K_{2}\right)$ as well as $\left(G, K_{1}\right)$ 
which is described in Lemma 2.2 satisfies the following property:

$\left(G, K_{2}\right)=\left(\prod_{i=1}^{c} S U\left(l_{i}^{\prime}+1\right) \times \prod_{j=1}^{d} S O\left(2 m_{j}^{\prime}+1\right) \times G_{2}^{\prime \prime}, \prod_{i=1}^{c} S\left(U\left(l_{i}^{\prime}\right) \times U(1)\right) \times \mathcal{S}_{2} \times G_{2}^{\prime \prime}\right)$,

where $\prod_{j=1}^{d} S O\left(2 m_{j}^{\prime}\right) \subset \mathcal{S}_{2} \subset \prod_{j=1}^{d} S\left(O(1) \times O\left(2 m_{j}^{\prime}\right)\right)$ and $G_{2}^{\prime \prime}$ is a product of connected, simple Lie groups and tori.

By the same argument of $[\mathbf{1 3}$, Section 6,7$]$ for $G / K_{1}^{o}$ and $G / K_{2}^{o}$ (where $K_{s}^{o}$ is the identity component of $K_{s}$ ), we have

$$
\left(G, K_{1}, K_{2}\right)=\left(\prod_{j=1}^{b} S O\left(2 m_{j}+1\right) \times \widehat{G}, \mathcal{S}_{1} \times \widehat{K_{1}}, \mathcal{S}_{2} \times \widehat{K_{2}}\right)
$$

such that $\left(\widehat{G}, \widehat{K_{1}}, \widehat{K_{2}}\right)$ is as follows:

$$
\begin{aligned}
& (a): \widehat{G}=\prod_{i=1}^{a} S U\left(l_{i}+1\right) \times S U(k) \times T^{1}, \\
& \widehat{K_{1}}=\widehat{K_{2}}=\prod_{i=1}^{a} S\left(U\left(l_{i}\right) \times U(1)\right) \times S U(k) \times T^{1}, \\
& \text { where } \operatorname{dim} G / K_{s}=2 n-2 k \text { and } k \geq 1\left(k_{1}=k_{2}=k\right) ; \\
& (b): \widehat{G}=\prod_{i=1}^{a-1} S U\left(l_{i}+1\right) \times S U\left(k_{1}\right) \times S U\left(k_{2}\right) \times T^{1}, \\
& \widehat{K_{1}}=\prod_{i=1}^{a-1} S\left(U\left(l_{i}\right) \times U(1)\right) \times S U\left(k_{1}\right) \times S\left(U\left(k_{2}-1\right) \times U(1)\right) \times T^{1}, \\
& \widehat{K_{2}}=\prod_{i=1}^{a-1} S\left(U\left(l_{i}\right) \times U(1)\right) \times S\left(U\left(k_{1}-1\right) \times U(1)\right) \times S U\left(k_{2}\right) \times T^{1}, \\
& \text { where } \operatorname{dim} G / K_{s}=2 n-2 k_{s} \text { and } k_{s} \geq 1 ; \\
& (c): \widehat{G}=\prod_{i=1}^{a} S U\left(l_{i}+1\right) \times S O(2 k), \\
& \widehat{K_{1}}=\widehat{K_{2}}=\prod_{i=1}^{a} S\left(U\left(l_{i}\right) \times U(1)\right) \times S O(2 k), \\
& \text { where } \operatorname{dim} G / K_{s}=2 n-2 k \text { and } k \geq 2\left(k_{1}=k_{2}=k\right) .
\end{aligned}
$$

We call the above cases the case (1)-(a), (1)-(b) and (1)-(c), respectively.

Next, we consider the slice representation $\sigma_{s}$ for $s=1,2$. Because of Lemma 2.3 and 2.5 , we have $\sigma_{s}: K_{s}=K_{s}^{\prime} \times G_{s}^{\prime \prime} \rightarrow S O\left(2 k_{s}\right)$. Moreover, there are the following two cases:

\begin{tabular}{|c|c|c|}
\hline$G_{s}^{\prime \prime}$ & $\sigma_{s}\left(G_{s}^{\prime \prime}\right)$ & $\sigma_{s}\left(K_{s}^{\prime}\right)$ \\
\hline \hline$S U\left(k_{s}\right) \times T^{1}$ & $U\left(k_{s}\right) \subset O\left(2 k_{s}\right)$ & $Z\left(U\left(k_{s}\right)\right) \simeq T^{1} \subset U\left(k_{s}\right) \subset S O\left(2 k_{s}\right)$ \\
\hline$S O\left(2 k_{s}\right)$ & $S O\left(2 k_{s}\right)$ & $Z\left(S O\left(2 k_{s}\right)\right)=\left\{ \pm I_{2 k_{s}}\right\} \subset S O\left(2 k_{s}\right)$ \\
\hline
\end{tabular}

where the right list means $\sigma_{s}\left(K_{s}^{\prime}\right) \subset Z\left(U\left(k_{s}\right)\right)$ or $Z\left(S O\left(2 k_{s}\right)\right)$.

In order to classify the above each case, we analyze the followings: principal isotropy subgroups $K=\sigma_{1}^{-1}\left(S O\left(2 k_{1}-1\right)\right)=\sigma_{2}^{-1}\left(S O\left(2 k_{2}-1\right)\right)$; attaching maps $f: G / K=\partial X_{1} \rightarrow \partial X_{2}=G / K \in N(K ; G) / N(K ; G)^{o}$ (see Section 2.4); and constructions of $G$-manifolds as $M(f)=X_{1} \cup_{f} X_{2}$, where $X_{s}$ denotes a $G$-invariant tubular neighborhood of $G / K_{s}$ for $s=1,2$.

3.3. The case (1)-(a). In this subsection, we study the case (1)-(a), that is,

- $G=\prod_{j=1}^{b} S O\left(2 m_{j}+1\right) \times \prod_{i=1}^{a} S U\left(l_{i}+1\right) \times S U(k) \times T^{1}$,

- $K_{1}=\mathcal{S}_{1} \times \prod_{i=1}^{a} S\left(U\left(l_{i}\right) \times U(1)\right) \times S U(k) \times T^{1}$,

- $K_{2}=\mathcal{S}_{2} \times \prod_{i=1}^{a} S\left(U\left(l_{i}\right) \times U(1)\right) \times S U(k) \times T^{1}$

where $\prod_{j=1}^{b} S O\left(2 m_{j}\right) \subset \mathcal{S}_{s} \subset \prod_{j=1}^{b} S\left(O\left(2 m_{j}\right) \times O(1)\right), \operatorname{dim} G / K_{s}=2 n-2 k$, and $k \geq 1$ for $s=1,2$.

In order to know the precise structure of $K$, we analyze slice representations $\sigma_{s}: K_{s} \rightarrow S O(2 k)$. By Section 3.2, the slice representation $\sigma_{s}: K_{s} \rightarrow U(k) \subset$ 
$S O(2 k)$ satisfies the followings:

$$
\begin{aligned}
& \sigma_{s}\left(S\left(U\left(l_{i}\right) \times U(1)\right)\right)=\left\{I_{2 k}\right\} \text { or } Z(U(k))=T^{1} \subset U(k) \text { for all } i=1, \cdots, a ; \\
& \sigma_{s}\left(S U(k) \times T^{1}\right)=U(k),
\end{aligned}
$$

where $Z(U(k))=T^{1} \subset U(k)$ is the center of $U(k)$, i.e., the diagonal subgroup. Because $\sigma_{s}\left(\mathcal{S}_{s}^{o}\right)=\sigma_{s}\left(\prod_{j=1}^{b} S O\left(2 m_{j}\right)\right)=\{1\} \subset T^{1} \subset U(k)$ (by making use of Lemma 2.5) and $\mathcal{S}_{s} / \prod_{j=1}^{b} S O\left(2 m_{j}\right) \simeq \mathcal{A}_{s} \subset \prod_{j=1}^{b} \mathbb{Z}_{2}$ (i.e., $\mathcal{A}_{s}$ is a subgroup of $\prod_{j=1}^{b} \mathbb{Z}_{2}$ generated by antipodal involutions), we have that

$$
\sigma_{s}\left(\mathcal{S}_{s}\right)=\sigma_{\mathbb{C}}\left(\mathcal{A}_{s}\right) \subset\{ \pm 1\} \subset T^{1} \subset U(k),
$$

where $\sigma_{\mathbb{C}}: \mathcal{A}_{s} \rightarrow\{ \pm 1\}$. If $\sigma_{s}\left(\mathcal{S}_{s}\right)=\{1\}$ then we can apply the same argument in [13]. So we assume $\sigma_{s}\left(\mathcal{S}_{s}\right)=\{ \pm 1\}$.

Now, the principal isotropy subgroup $K$ is as follows $(X \equiv Y$ means that two groups $X$ and $Y$ are conjugate in $G$ ):

$$
\begin{aligned}
K & \equiv \sigma_{1}^{-1}(U(k-1))=\left\{\left(A,\left(t_{1}, \cdots, t_{a}\right),\left(\begin{array}{cc}
X & 0 \\
0 & x
\end{array}\right), t\right) \mid \sigma_{1}(A) x t_{1}^{\alpha_{1}} \cdots t_{a}^{\alpha_{a}} t^{\alpha}=1\right\} \\
& \equiv \sigma_{2}^{-1}(U(k-1))=\left\{\left(B,\left(t_{1}, \cdots, t_{a}\right),\left(\begin{array}{cc}
Y & 0 \\
0 & y
\end{array}\right), t\right) \mid \sigma_{2}(B) y t_{1}^{\beta_{1}} \cdots t_{a}^{\beta_{a}} t^{\beta}=1\right\},
\end{aligned}
$$

where $A \in \mathcal{S}_{1}, B \in \mathcal{S}_{2} ; X, Y \in U(k-1), x, y \in T^{1}$ such that $x \operatorname{det} X=y \operatorname{det} Y=1$; and

$$
\left(t_{1}, \cdots, t_{a}\right)=\left(\left(\begin{array}{cc}
U_{1} & 0 \\
0 & t_{1}
\end{array}\right), \cdots,\left(\begin{array}{cc}
U_{a} & 0 \\
0 & t_{a}
\end{array}\right)\right) \in \prod_{i=1}^{a} S\left(U\left(l_{i}\right) \times U(1)\right) .
$$

First we take $A=B=e \in \mathcal{S}_{1} \cap \mathcal{S}_{2}$ (the identity elements in $\prod_{j=1}^{b} S O\left(2 m_{j}+1\right)$ ). Then $\sigma_{1}(A)=1=\sigma_{2}(B)$. Hence, by using the the same argument of $[\mathbf{1 3}$, Section 7.1] for $A=B=e$, we have that

$$
\alpha_{i}=\beta_{i} \in \mathbb{Z} \text { for } i=1, \cdots, a, \quad \text { and } \quad \alpha=\beta \in \mathbb{N} .
$$

Moreover, if $k=1$ then we can take $\alpha=\beta=1$ (up to essential isomorphism). Next, we consider the following part in $K$ (if $k \geq 2$ ):

$$
\begin{aligned}
\mathcal{R}_{1} & =\left\{\left(A, e,\left(\begin{array}{cc}
J & 0 \\
0 & \sigma_{1}(A)
\end{array}\right), 1\right) \mid A \in \mathcal{S}_{1}\right\} \\
& \equiv\left\{\left(B, e,\left(\begin{array}{cc}
J & 0 \\
0 & \sigma_{2}(B)
\end{array}\right), 1\right) \mid B \in \mathcal{S}_{2}\right\}=\mathcal{R}_{2},
\end{aligned}
$$

where $e \in \prod_{i=1}^{a} S\left(U\left(l_{i}\right) \times U(1)\right)$ is the identity element and $J \in U(k-1)$ such that $J=I_{k-1}$ (if $\sigma_{s}\left(C_{s}\right)=1$ ) or $\operatorname{det} J=-1$ with $J^{2}=I_{k-1}\left(\right.$ if $\sigma_{s}\left(C_{s}\right)=-1$ ) for $s=1,2$ and $C_{1}=A, C_{2}=B$. For $\mathcal{R}_{1}$ and $\mathcal{R}_{2}$, we can easily show the following isomorphisms (not identity in $G$ ):

$$
\mathcal{S}_{1} \simeq \mathcal{R}_{1}, \quad \mathcal{S}_{2} \simeq \mathcal{R}_{2} .
$$

Because $\mathcal{R}_{1} \equiv \mathcal{R}_{2}$, we see that

$$
\mathcal{S}_{1}=\mathcal{S}_{2} \subset K_{1} \cap K_{2}
$$


by the definition of $\mathcal{S}_{s}$, i.e., we can regard $K_{1}=K_{2}$ in $G$. If $k=1$, then we can apply the same above argument by taking the set $\left\{\left(A, e, \sigma_{1}(A)\right)\right\} \equiv\left\{\left(B, e, \sigma_{2}(B)\right)\right\}$, and we can regard $K_{1}=K_{2}$. Therefore, by using the above argument, we have $\sigma_{1}=\sigma_{2}$. Moreover, $K$ is as follows:

$$
K=\left\{\left(A,\left(t_{1}, \cdots, t_{a}\right),\left(\begin{array}{cc}
X & 0 \\
0 & x
\end{array}\right), t\right) \mid \sigma_{1}(A) x t_{1}^{\alpha_{1}} \cdots t_{a}^{\alpha_{a}} t^{\alpha}=1\right\}
$$

As the result of the above argument, the tubular neighborhood $X_{s}=G \times_{K_{s}} D^{2 k}$ $(s=1,2)$ is equivariantly diffeomorphic to the following manifold:

$$
\prod_{j=1}^{b} S^{2 m_{j}} \times_{\mathcal{A}}\left(\prod_{i=1}^{a} S^{2 l_{i}+1} \times_{T^{a}} D\left(\mathbb{C}_{\mathfrak{a}}^{k}\right)\right),
$$

where $T^{a}$ acts on $\prod_{i=1}^{a} S^{2 l_{i}+1}$ naturally, and on $D\left(\mathbb{C}_{\mathfrak{a}}^{k}\right)$ by $\mathfrak{a}:\left(t_{1}, \cdots, t_{a}\right) \mapsto$ $t_{1}^{\alpha_{1}} \cdots t_{a}^{\alpha_{a}} ;$ and $\mathcal{A}=\mathcal{A}_{1}=\mathcal{A}_{2}\left(\simeq \mathcal{S}_{1} / \mathcal{S}_{1}^{o}=\mathcal{S}_{2} / \mathcal{S}_{2}^{o}\right)$ acts on $\prod_{j=1}^{b} S^{2 m_{j}}$ as the subgroup of $\prod_{j=1}^{b} \mathbb{Z}_{2}$, and on $D\left(\mathbb{C}_{\mathfrak{a}}^{k}\right)$ by $\sigma_{\mathbb{C}}: \mathcal{A} \rightarrow\{ \pm 1\}$ (induced by $\sigma_{1}$ ). Here, $G=\prod_{j=1}^{b} S O\left(2 m_{j}+1\right) \times \prod_{i=1}^{a} S U\left(l_{i}+1\right) \times S U(k) \times T^{1}$ acts on this manifold as follows: $\prod_{j=1}^{b} S O\left(2 m_{j}+1\right)$ acts on $\prod_{j=1}^{b} S^{2 m_{j}}$ naturally; $\prod_{i=1}^{a} S U\left(l_{i}+1\right)$ acts on $\prod_{i=1}^{a} S^{2 l_{i}+1}$ naturally; and $S U(k) \times T^{1}$ acts on $D\left(\mathbb{C}_{\mathfrak{a}}^{k}\right)$ by $(A, t) \mapsto A t^{\alpha} \in U(k)$.

Next, we analyze attaching maps $f: \partial X_{1} \rightarrow \partial X_{2}$. Because of $\alpha \in \mathbb{N}$, we have

$$
N(K ; G) / N(K ; G)^{o} \simeq \prod_{j=1}^{b} \mathbb{Z}_{2} \times \prod_{i=1}^{a} W_{l_{i}+1},
$$

where $\prod_{j=1}^{b} S\left(O\left(2 m_{j}\right) \times O(1)\right) / S O\left(2 m_{j}\right) \simeq \prod_{j=1}^{b} \mathbb{Z}_{2}$ and

$$
W_{l_{i}+1}=\left\{\begin{array}{cl}
\left\{I_{l_{i}+1}\right\} & \text { if } l_{i} \geq 2 \text { or } \alpha_{i} \neq 0 \\
S_{2} & \text { if } l_{i}=1 \text { and } \alpha_{i}=0 .
\end{array}\right.
$$

Here, the above $S_{2}\left(\simeq \mathbb{Z}_{2}\right)$ is the Weyl group of $S U(2)$. Therefore, by the same argument as [13, Section 8.2], we can show that $I \circ f: \partial X_{1} \rightarrow \partial X_{1}$ is extendable to the equivariant map $X_{1} \rightarrow X_{1}$, where $I: G / K=\partial X_{2} \rightarrow \partial X_{1}=G / K$ is the identity attaching map. Hence, $M(f) \cong M(I)$ for all attaching maps $f$ by the Uchida's criterion (2). As the result, we have that if the case (1) holds for $(M, G)$, then such $(M, G)$ is only determined by the representations $\mathfrak{a}: T^{a} \rightarrow T^{1}$ and $\sigma_{\mathbb{C}}: \mathcal{A} \rightarrow\{ \pm 1\}$ (up to essential isomorphism), i.e., if we fix the representations $\mathfrak{a}$ and $\sigma_{\mathbb{C}}$, then $(M, G)$ is unique up to essential isomorphism. Thus, we have that such manifold $M$ and $G$ are as follows (up to essential isomorphism):

$$
\begin{aligned}
& M=\prod_{j=1}^{b} S^{2 m_{j}} \times_{\mathcal{A}}\left(\prod_{i=1}^{a} S^{2 l_{i}+1} \times_{T^{a}} S\left(\mathbb{C}_{\mathfrak{a}}^{k} \oplus \mathbb{R}\right)\right), \\
& G=\prod_{j=1}^{b} S O\left(2 m_{j}+1\right) \times \prod_{i=1}^{a} S U\left(l_{i}+1\right) \times U(k),
\end{aligned}
$$

by computing the orbits $G / K, G / K_{1}$ and $G / K_{2}$. This corresponds with the first case of Theorem 3.1. 
3.4. The case (1)-(b). In this subsection, we study the case (1)-(b), that is,

- $G=\prod_{j=1}^{b} S O\left(2 m_{j}+1\right) \times \prod_{i=1}^{a-1} S U\left(l_{i}+1\right) \times S U\left(k_{1}\right) \times S U\left(k_{2}\right) \times T^{1}$,

- $K_{1}=\mathcal{S}_{1} \times \prod_{i=1}^{a-1} S\left(U\left(l_{i}\right) \times U(1)\right) \times S U\left(k_{1}\right) \times S\left(U\left(k_{2}-1\right) \times U(1)\right) \times T^{1}$,

- $K_{2}=\mathcal{S}_{2} \times \prod_{i=1}^{a-1} S\left(U\left(l_{i}\right) \times U(1)\right) \times S\left(U\left(k_{1}-1\right) \times U(1)\right) \times S U\left(k_{2}\right) \times T^{1}$, where $\prod_{j=1}^{b} S O\left(2 m_{j}\right) \subset \mathcal{S}_{s} \subset \prod_{j=1}^{b} S\left(O\left(2 m_{j}\right) \times O(1)\right), \operatorname{dim} G / K_{s}=2 n-2 k_{s}$ and $k_{s} \geq 1$ for $s=1,2$.

In order to know the precise structure of $K$, we analyze the slice representation $\sigma_{s}: K_{s} \rightarrow S O\left(2 k_{s}\right)$. The following property can be proved similarly to that of the case (1)-(a) (see Section 3.2, 3.3):

$$
\begin{aligned}
& \sigma_{s}\left(S\left(U\left(l_{i}\right) \times U(1)\right)\right)=\left\{I_{2 k_{s}}\right\} \text { or } Z\left(U\left(k_{s}\right)\right) \subset U\left(k_{s}\right) \text { for all } i=1, \cdots, a-1 ; \\
& \sigma_{s}\left(S\left(U\left(k_{r}-1\right) \times U(1)\right)\right)=\left\{I_{2 k_{s}}\right\} \text { or } Z\left(U\left(k_{s}\right)\right) \subset U\left(k_{s}\right) ; \\
& \sigma_{s}\left(S U\left(k_{s}\right) \times T^{1}\right)=U\left(k_{s}\right) ; \\
& \sigma_{s}\left(\mathcal{S}_{s}\right)=\sigma_{\mathbb{C}}\left(\mathcal{A}_{s}\right) \subset\{ \pm 1\},
\end{aligned}
$$

where $s+r=3$ and $s, r \geq 1$. Therefore, $K$ satisfies that

$$
\begin{aligned}
K & \equiv \sigma_{1}^{-1}\left(U\left(k_{1}-1\right)\right) \\
& =\left\{\left(A,\left(t_{1}, \cdots, t_{a-1}\right),\left(\begin{array}{cc}
C & 0 \\
0 & c
\end{array}\right),\left(\begin{array}{cc}
X & 0 \\
0 & x
\end{array}\right), t\right) \mid \sigma_{1}(A)=t_{1}^{\alpha_{1}} \cdots t_{a-1}^{\alpha_{a-1}} x^{\alpha_{a}} t^{\alpha} c\right\} \\
& \equiv \sigma_{2}^{-1}\left(U\left(k_{2}-1\right)\right) \\
& =\left\{\left(B,\left(t_{1}, \cdots, t_{a-1}\right),\left(\begin{array}{cc}
Y & 0 \\
0 & y
\end{array}\right),\left(\begin{array}{cc}
D & 0 \\
0 & d
\end{array}\right), t\right) \mid \sigma_{2}(B)=t_{1}^{\beta_{1}} \cdots t_{a-1}^{\beta_{a-1}} y^{\beta_{a}} t^{\beta} d\right\},
\end{aligned}
$$

where $A \in \mathcal{S}_{1}, B \in \mathcal{S}_{2},\left(t_{1}, \cdots, t_{a-1}\right) \in \prod_{i=1}^{a-1} S\left(U\left(l_{i}\right) \times U(1)\right)$, and $C, Y \in$ $U\left(k_{1}-1\right), D, X \in U\left(k_{2}-1\right), x, y, c, d \in T^{1}$ such that $x \operatorname{det} X=y \operatorname{det} Y=$ $c \operatorname{det} C=d \operatorname{det} D=1$.

By the same argument as $\left[\mathbf{1 3}\right.$, Section 7.2] for $\sigma_{1}(A)=1=\sigma_{2}(B)$, we see that $\alpha_{i}=\beta_{i} \in \mathbb{Z}$ for $i=1, \cdots, a-1, \quad$ and $\quad \alpha_{a}=\beta_{a}=1 \quad$ and $\quad \alpha=\beta \in \mathbb{N}$.

Moreover, if $k_{1}=1$ or $k_{2}=1$, then we have that $\alpha=1$ or $\beta=1$, respectively. Using the method similar to that demonstrated in Section 3.3, we can also prove

$$
\mathcal{S}_{1}=\mathcal{S}_{2} \subset K_{1} \cap K_{2},
$$

and $\left.\sigma_{1}\right|_{\mathcal{S}_{1}}=\left.\sigma_{2}\right|_{\mathcal{S}_{2}}$ (i.e., the restricted representations are the same representations). Hence, $K$ is as follows:

$K=\left\{\left(A,\left(t_{1}, \cdots, t_{a-1}\right),\left(\begin{array}{cc}Y & 0 \\ 0 & y\end{array}\right),\left(\begin{array}{cc}X & 0 \\ 0 & x\end{array}\right), t\right) \mid \sigma_{1}(A) t_{1}^{\alpha_{1}} \cdots t_{a-1}^{\alpha_{a-1}} x y t^{\alpha}=1\right\}$.

As the result of the above argument, the tubular neighborhood $X_{s}=G \times_{K_{s}}$ $D^{2 k_{s}}(s=1,2)$ is equivariantly diffeomorphic to the following manifold:

$$
\prod_{j=1}^{b} S^{2 m_{j}} \times_{\mathcal{A}}\left(\prod_{i=1}^{a-1} S^{2 l_{i}+1} \times_{T^{a-1}} D\left(\mathbb{C}_{\mathfrak{b}}^{k_{s}}\right)\right) \times \mathbb{C} P\left(k_{r}-1\right),
$$

where $T^{a-1}$ acts on $\prod_{i=1}^{a-1} S^{2 l_{i}+1}$ naturally and on $D\left(\mathbb{C}_{\mathfrak{b}}^{k_{s}}\right)$ by $\mathfrak{b}:\left(t_{1}, \cdots, t_{a-1}\right) \mapsto$ $t_{1}^{\alpha_{1}} \cdots t_{a-1}^{\alpha_{a-1}}, \mathcal{A}\left(=\mathcal{A}_{s} \simeq \mathcal{S}_{s} / \mathcal{S}_{s}^{o}\right)$ acts on $\prod_{j=1}^{b} S^{2 m_{j}}$ as the subgroup of $\prod_{j=1}^{b} \mathbb{Z}_{2}$ and on $D\left(\mathbb{C}_{\mathfrak{b}}^{k_{s}}\right)$ by $\sigma_{\mathbb{C}}: \mathcal{A} \rightarrow\{ \pm 1\}$, and $s+r=3$ for $s, r \geq 1$. Here, $G=$ 
$\prod_{j=1}^{b} S O\left(2 m_{j}+1\right) \times \prod_{i=1}^{a-1} S U\left(l_{i}+1\right) \times S U\left(k_{s}\right) \times T^{1} \times S U\left(k_{r}\right)$ acts on this manifold as follows: $\prod_{j=1}^{b} S O\left(2 m_{j}+1\right)$ acts on $\prod_{j=1}^{b} S^{2 m_{j}}$ naturally; $\prod_{i=1}^{a-1} S U\left(l_{i}+1\right)$ acts on $\prod_{i=1}^{a-1} S^{2 l_{i}+1}$ naturally; $S U\left(k_{s}\right) \times T^{1}$ acts on $D\left(\mathbb{C}_{\mathfrak{b}}^{k_{s}}\right)$ by $(A, t) \mapsto A t^{\alpha}$; and $S U\left(k_{r}\right)$ acts on $\mathbb{C} P\left(k_{r}-1\right)$ naturally.

Next, we analyze attaching maps $f: \partial X_{1} \rightarrow \partial X_{2}$. In this case, we have

$$
N(K ; G) / N(K ; G)^{o} \simeq \prod_{j=1}^{b} \mathbb{Z}_{2} \times \prod_{i=1}^{a-1} W_{l_{i}+1}
$$

where $\prod_{j=1}^{b} S\left(O\left(2 m_{j}\right) \times O(1)\right) / S O\left(2 m_{j}\right) \simeq \prod_{j=1}^{b} \mathbb{Z}_{2}$ and

$$
W_{l_{i}+1}=\left\{\begin{array}{cl}
\left\{I_{l_{i}+1}\right\} & \text { if } l_{i} \geq 2 \text { or } \alpha_{i} \neq 0 \\
S_{2} & \text { if } l_{i}=1 \text { and } \alpha_{i}=0
\end{array}\right.
$$

This is the same as the case (1)-(a). Therefore, we can show the Uchida's criterion (2) for $I \circ f$. Hence, by using the similar argument to that demonstrated in Section 3.3 , we have that $(M, G)$ which satisfies the case (1)-(b) is unique up to essential isomorphism if we fix the representations $\mathfrak{b}$ and $\sigma_{\mathbb{C}}$. Thus, we have that $M$ and $G$ in the case (1)-(b) are as follows (up to essential isomorphism):

$$
\begin{aligned}
& M=\prod_{j=1}^{b} S^{2 m_{j}} \times_{\mathcal{A}}\left(\prod_{i=1}^{a-1} S^{2 l_{i}+1} \times_{T^{a-1}} P\left(\mathbb{C}_{\mathfrak{b}}^{k_{1}} \oplus \mathbb{C}^{k_{2}}\right)\right), \\
& G=\prod_{j=1}^{b} S O\left(2 m_{j}+1\right) \times \prod_{i=1}^{a-1} S U\left(l_{i}+1\right) \times S\left(U\left(k_{1}\right) \times U\left(k_{2}\right)\right) .
\end{aligned}
$$

This corresponds with the second case of Theorem 3.1.

3.5. The case (1)-(c). In this subsection, we study the case (1)-(c), that is,

- $G=\prod_{j=1}^{b} S O\left(2 m_{j}+1\right) \times \prod_{i=1}^{a} S U\left(l_{i}+1\right) \times S O(2 k)$,

- $K_{1}=\mathcal{S}_{1} \times \prod_{i=1}^{a} S\left(U\left(l_{i}\right) \times U(1)\right) \times S O(2 k)$,

- $K_{2}=\mathcal{S}_{2} \times \prod_{i=1}^{a} S\left(U\left(l_{i}\right) \times U(1)\right) \times S O(2 k)$,

where $\prod_{j=1}^{b} S O\left(2 m_{j}\right) \subset \mathcal{S}_{s} \subset \prod_{j=1}^{b} S\left(O\left(2 m_{j}\right) \times O(1)\right), \operatorname{dim} G / K_{s}=2 n-2 k$ and $k \geq 2$ for $s=1,2$.

In order to know the precise structure of $K$, we analyze the slice representation $\sigma_{s}: K_{s} \rightarrow S O(2 k)$ as well as the cases (1) and (2). By Section 3.2, we have the followings for the slice representation $\sigma_{s}: K_{s} \rightarrow S O(2 k)$ :

$$
\begin{aligned}
& \sigma_{s}\left(S\left(U\left(l_{i}\right) \times U(1)\right)\right)=\left\{I_{2 k}\right\} \subset S O(2 k) \text { for all } i=1, \cdots, a ; \\
& \sigma_{s}(S O(2 k))=S O(2 k) ; \\
& \sigma_{s}\left(\mathcal{S}_{s}\right)=\sigma_{\mathbb{R}}\left(\mathcal{A}_{s}\right) \subset\left\{ \pm I_{2 k}\right\} .
\end{aligned}
$$

Therefore, we have that $K$ is the following subgroup:

$$
\begin{aligned}
K & \equiv \sigma_{1}^{-1}(S O(2 k-1))=\left\{\left(A,\left(t_{1}, \cdots, t_{a}\right),\left(\begin{array}{cc}
E & 0 \\
0 & e
\end{array}\right)\right) \mid \sigma_{1}(A) e=1\right\} \\
& \equiv \sigma_{2}^{-1}(S O(2 k-1))=\left\{\left(B,\left(t_{1}, \cdots, t_{a}\right),\left(\begin{array}{cc}
F & 0 \\
0 & f
\end{array}\right)\right) \mid \sigma_{2}(B) f=1\right\},
\end{aligned}
$$


where $A \in \mathcal{S}_{1}, B \in \mathcal{S}_{2},\left(t_{1}, \cdots, t_{a}\right) \in \prod_{i=1}^{a} S\left(U\left(l_{i}\right) \times U(1)\right)$, and $E, F \in O(2 k-1)$, $e, f \in O(1)$ such that $e \operatorname{det} E=f \operatorname{det} F=1$.

Next, we consider the following part in $K$ :

$$
\begin{aligned}
\mathcal{R}_{1} & =\left\{\left(A,(1, \cdots, 1),\left(\begin{array}{cc}
\sigma_{1}(A) I_{2 k-1} & 0 \\
0 & \sigma_{1}(A)
\end{array}\right)\right) \mid \sigma_{1}(A)= \pm 1\right\} \\
& \equiv\left\{\left(B,(1, \cdots, 1),\left(\begin{array}{cc}
\sigma_{2}(B) I_{2 k-1} & 0 \\
0 & \sigma_{2}(B)
\end{array}\right)\right) \mid \sigma_{2}(B)= \pm 1\right\}=\mathcal{R}_{2} .
\end{aligned}
$$

By using $\mathcal{R}_{1}, \mathcal{R}_{2}$ and the method similar to that demonstrated in Section 3.3 (the case (1)-(a)), we can regard $K_{1}=K_{2}$ in $G$ and $\sigma_{1}=\sigma_{2}$. Hence, $K$ is as follows:

$K=\prod_{i=1}^{a} S\left(U\left(l_{i}\right) \times U(1)\right) \times\left\{\left(A,\left(\begin{array}{cc}X & 0 \\ 0 & x\end{array}\right)\right) \in \mathcal{S}_{1} \times S(O(2 k-1) \times O(1)) \mid \sigma_{1}(A) x=1\right\}$

As the result of the above argument, the tubular neighborhood $X_{s}=G \times_{K_{s}} D^{2 k}$ $(s=1,2)$ is equivariantly diffeomorphic to the following manifold:

$$
\prod_{i=1}^{a} \mathbb{C} P\left(l_{i}\right) \times\left(\prod_{j=1}^{b} S^{2 m_{j}} \times_{\mathcal{A}} D\left(\mathbb{R}^{2 k}\right)\right),
$$

where $\mathcal{A}\left(=\mathcal{A}_{s} \simeq \mathcal{S}_{s} / \mathcal{S}_{s}^{o}\right)$ acts on $\prod_{j=1}^{b} S^{2 m_{j}}$ as the subgroup of $\prod_{j=1}^{b} \mathbb{Z}_{2}$ and on $D\left(\mathbb{R}^{2 k}\right)$ by $\sigma_{\mathbb{R}}: \mathcal{A} \rightarrow\left\{ \pm I_{2 k}\right\}$. Here, $G=\prod_{j=1}^{b} S O\left(2 m_{j}+1\right) \times \prod_{i=1}^{a} S U\left(l_{i}+\right.$ 1) $\times S O(2 k)$ acts on this manifold as follows: $\prod_{i=1}^{a} S U\left(l_{i}+1\right)$ acts on $\prod_{i=1}^{a} \mathbb{C} P\left(l_{i}\right)$ naturally; $\prod_{j=1}^{b} S O\left(2 m_{j}+1\right)$ acts on $\prod_{j=1}^{b} S^{2 m_{j}}$ naturally; and $S O(2 k)$ acts on $D\left(\mathbb{R}^{2 k}\right)$ naturally.

Next, we analyze attaching maps $f: \partial X_{1} \rightarrow \partial X_{2}$. In this case, we have

$$
N(K ; G) / N(K ; G)^{o} \simeq \prod_{j=1}^{b} \mathbb{Z}_{2} \times \prod_{i=1}^{a} W_{l_{i}} \times W
$$

where $W=S(O(2 k-1) \times O(1)) / S O(2 k-1)=\left\{I_{2 k},-I_{2 k}\right\} \simeq \mathbb{Z}_{2}$, and

$$
W_{l_{i}+1}=\left\{\begin{array}{cl}
\left\{I_{l_{i}+1}\right\} & \text { if } l_{i} \geq 2 \text { or } \alpha_{i} \neq 0 \\
S_{2} & \text { if } l_{i}=1 \text { and } \alpha_{i}=0 .
\end{array}\right.
$$

Therefore, by the same argument of [13, Section 8.4], we can show the Uchida's criterion (2) for $I \circ f$. Hence, by using the similar argument to that demonstrated in Section 3.3, we have that $(M, G)$ which satisfies the case (1)-(c) is unique up to essential isomorphism if we fix the representation $\sigma_{\mathbb{R}}$. Thus, we have that $M$ and $G$ in the case (1)-(c) are as follows (up to essential isomorphism):

$$
\begin{aligned}
M & =\prod_{j=1}^{b} S^{2 m_{j}} \times_{\mathcal{A}}\left(\prod_{i=1}^{a} \mathbb{C} P\left(l_{i}\right) \times S\left(\mathbb{R}^{2 k} \oplus \mathbb{R}\right)\right), \\
G & =\prod_{j=1}^{b} S O\left(2 m_{j}+1\right) \times \prod_{i=1}^{a} S U\left(l_{i}+1\right) \times S O(2 k) .
\end{aligned}
$$

This corresponds with the third case of Theorem 3.1. 


\section{Preliminary for the cases (2) and (3)}

In this section, we prepare to classify the other cases, i.e., $G / K_{1}$ is a torus manifold but $G / K_{2}$ is not a torus manifold (the cases (2) and (3)). Henceforth, we assume that $G / K_{2}$ is not a torus manifold. In this case, $G / K_{2} \cap M^{T}=\emptyset$, by using Lemma 2.1. It follows that $T \subset G$ (a maximal torus) but $T \not \subset K_{2}^{o}$, i.e., rank $G>\operatorname{rank} K_{2}^{o}$.

We first prove that $\operatorname{dim} G / K_{2}^{o}=\operatorname{dim} G / K_{2}$ is odd. Because $G / K_{1}$ is a torus manifold, we can apply the same argument which is demonstrated in Section 3 to get $K$ (also see Section 4.1). By using $K$ 's in Section 3, we can easily show the following formula:

$$
\operatorname{rank} G=\operatorname{rank} K_{1}^{o}=\operatorname{rank} K^{o}+1 .
$$

Let $T^{\prime} \subset K^{o}$ be a maximal torus such that $T^{\prime} \subset T$. If $T^{\prime}$ is not a maximal torus in $K_{2}^{o}$, then we have rank $K_{2}^{o}=\operatorname{rank} K^{o}+1$ by using $T^{\prime} \subset K^{o} \subset K_{2}^{o} \subset G$ and the above formula. However, this gives a contradiction to $\operatorname{rank} G>\operatorname{rank} K_{2}^{o}$. Therefore, we have $\operatorname{rank} K_{2}^{o}=\operatorname{rank} K^{o}$. Due to [7, Theorem 1.1], we also have $\operatorname{dim} K_{2}^{o} / K^{o}$ is even. Thus, by considering the fibration: $K_{2}^{o} / K^{o} \rightarrow G / K^{o} \rightarrow G / K_{2}^{o}$ and $\operatorname{dim} G / K^{o}=2 n-1$, we have that $\operatorname{dim} G / K_{2}^{o}=\operatorname{dim} G / K_{2}$ is odd. Hence, we can put

$$
\operatorname{dim} G / K_{2}=2 n-2 k_{2}+1
$$

for $k_{2} \geq 1$. Remark that if $k_{2}=1$ then this is in the case (2): $G / K_{2}$ is an exceptional orbit, otherwise this is in the case (3): $G / K_{2}$ is a singular orbit.

Since our $G$-action on $M$ has codimension 1 orbits, we have that

$$
K_{2} / K \cong S^{2 k_{2}-2}
$$

in each case.

In summary, we have the following lemma.

Lemma 4.1. Suppose a torus manifold $(M, T)$ has codimension 1 extended $G$-actions. If $G / K_{2}$ is not a torus manifold, then

$$
\operatorname{dim} G / K_{2}=2 n-2 k_{2}+1, \quad K_{2} / K \cong S^{2 k_{2}-2},
$$

for $k_{2} \geq 1$.

4.1. Structures of $G / K_{1}$ and their tubular neighborhoods of the cases (2) and (3). The main part to classify the cases (2) and (3) is to determine the group $K_{2}$ and its inclusion $K_{2} \subset G$. From the next section, We will determine $K_{2} \subset G$ by making use of the relation $K \subset K_{2} \subset G$ and the classification result of the transitive action on $S^{2 k_{2}-2}$. In this subsection, we recall $G, K_{1}$ and $K$; and in the next subsection, we give an important remark for the attaching map.

Let $\operatorname{dim} G / K_{1}=2 n-2 k_{1}$ for $k_{1} \geq 1$. By Lemma 2.2, 2.3 and Remark 2.4, we have $\left(G, K_{1}\right)$ as the following two cases:

(a): $G=\prod_{j=1}^{b} S O\left(2 m_{j}+1\right) \times \prod_{i=1}^{a} S U\left(l_{i}+1\right) \times S U\left(k_{1}\right) \times T^{1}$, and $K_{1}=$ $\mathcal{S}_{1} \times \prod_{i=1}^{a} S\left(U\left(l_{i}\right) \times U(1)\right) \times S U\left(k_{1}\right) \times T^{1}$ for $k_{1} \geq 1$;

(b): $G=\prod_{j=1}^{b} S O\left(2 m_{j}+1\right) \times \prod_{i=1}^{a} S U\left(l_{i}+1\right) \times S O\left(2 k_{1}\right)$, and $K_{1}=\mathcal{S}_{1} \times$ $\prod_{i=1}^{a} S\left(U\left(l_{i}\right) \times U(1)\right) \times S O\left(2 k_{1}\right)$ for $k_{1} \geq 2$.

We call them the case (a) and (b) respectively. 
4.1.1. The case (a). For the principal isotropy subgroup $K$ of the case (a), we can easily show the following by using the same argument in Section 3.3:

$$
K=\left\{\left(A,\left(t_{1}, \cdots, t_{a}\right),\left(\begin{array}{cc}
X & 0 \\
0 & x
\end{array}\right), t\right) \mid \sigma_{1}(A) x t_{1}^{\alpha_{1}} \cdots t_{a}^{\alpha_{a}} t^{\alpha}=1\right\},
$$

where $A \in \mathcal{S}_{1},\left(t_{1}, \cdots, t_{a}\right) \in \prod_{i=1}^{a} S\left(U\left(l_{i}\right) \times U(1)\right), t \in T^{1}$ and $X \in U\left(k_{1}-1\right)$ such that $x \operatorname{det} X=1$. Here, $\sigma_{1}(A) \subset\{ \pm 1\} \subset S^{1}$ and $\left(\alpha_{1}, \cdots, \alpha_{a}, \alpha\right) \in \mathbb{Z}^{a} \times \mathbb{N}$. If $k_{1}=$ 1 , then we can take $\alpha=1$. Therefore, a tubular neighborhood $X_{1}=G \times_{K_{1}} D^{2 k_{1}}$ of the case (a) is equivariantly diffeomorphic to the following manifold defined in $(3.1)$ :

$$
\prod_{j=1}^{b} S^{2 m_{j}} \times_{\mathcal{A}_{1}}\left(\prod_{i=1}^{a} S^{2 l_{i}+1} \times_{T^{a}} D\left(\mathbb{C}_{\mathfrak{c}}^{k_{1}}\right)\right)
$$

Remark that from this section we write the representation $\mathfrak{a}: T^{a} \rightarrow S^{1}$ as $\mathfrak{c}$.

4.1.2. The case (b). For the principal isotropy subgroup $K$ of the case (b), we can easily show the following by using the same argument in Section 3.5:

$$
\begin{aligned}
K & =\prod_{i=1}^{a} S\left(U\left(l_{i}\right) \times U(1)\right) \\
& \times\left\{\left(A,\left(\begin{array}{cc}
X & 0 \\
0 & x
\end{array}\right)\right) \in \mathcal{S}_{1} \times S\left(O\left(2 k_{1}-1\right) \times O(1)\right) \mid \sigma_{1}(A) x=1\right\} .
\end{aligned}
$$

Hence, a tubular neighborhood $X_{1}=G \times_{K_{1}} D^{2 k_{1}}$ of the case (b) is equivariantly diffeomorphic to the following manifold defined in (3.2):

$$
\prod_{i=1}^{a} \mathbb{C} P\left(l_{i}\right) \times\left(\prod_{j=1}^{b} S^{2 m_{j}} \times \mathcal{A}_{1} D\left(\mathbb{R}^{2 k_{1}}\right)\right) .
$$

4.2. Important remark for the attaching maps of the cases (2) and (3). Before we go to the next section, we give the following important remark for the attaching maps $f: G / K=\partial X_{1} \rightarrow \partial X_{2}=G / K$.

REMARK 4.2. In each above case (a) and (b), we see that $N(K ; G) / N(K ; G)^{o}$ is the same as one of the cases (1)-(a), (1)-(b) and (1)-(c). Therefore, we have already proved the Uchida's criterion (2) for $I \circ f$ in Section 3, that is, $I \circ f: \partial X_{1} \rightarrow \partial X_{1}$ is extendable to the equivariant map $X_{1} \rightarrow X_{1}$. Hence, once we know $X_{1}$ and $X_{2}$ for the cases (2) and (3), then a $G$-diffeomorphism $M(f) \cong M(I)$ always holds, i.e., the constructing manifold $X_{1} \cup X_{2}$ is unique. It follows that, for the cases (2) and (3), we may only analyse the structure of $X_{2}=G \times_{K_{2}} D^{2 k_{2}-1}$.

In the next six sections (the case (2) in Section 5, 6, 7; the case (3) in Section $8,9,10)$, we will analyze $K_{2}$ and its slice representation $\sigma_{2}: K_{2} \rightarrow O\left(2 k_{2}-1\right)$ of the cases (2) and (3).

\section{Main theorem and remarks of the case (2)}

The goal of this section is to state the main theorem and give some remarks of the case (2). In this section and the next two sections, we assume that the other singular orbit $G / K_{2}$ is an exceptional orbit, i.e., $\operatorname{dim} G / K_{2}=\operatorname{dim} G / K=2 n-1$. 
Then we have $k_{2}=1$ and $K_{2} / K \cong S^{0}$ by Lemma 4.1. Due to [1, 3.2 Theorem (ii) in Chapter IV], we have that $K$ is a normal subgroup of $K_{2}$, i.e.,

$$
K \subset K_{2} \subset N(K ; G),
$$

where $N(K ; G)$ is the normalizer of $K$ in $G$.

5.1. Main theorem of the case (2). Before we state the main theorem, we prepare some notations (also see Section 3.1). Let $\mathcal{A}$ be a subgroup of $\prod_{j=1}^{b} \mathbb{Z}_{2}$, where $\prod_{j=1}^{b} \mathbb{Z}_{2}$ is generated by the antipodal involutions on $S^{2 m_{j}}$ for $j=1, \cdots, b$. The subgroup $\Delta$ in $S^{1} \times O(1)$ (resp. in $S O\left(2 k_{1}\right) \times O(1)$ ) denotes the diagonal subgroup $\{(1,1),(-1,-1)\}$ (resp. $\left.\left\{\left(I_{2 k_{1}}, 1\right),\left(-I_{2 k_{1}},-1\right)\right\}\right)$, and the subgroup $O(1)$ in $S^{1} \times O(1)\left(\right.$ resp. in $\left.S O\left(2 k_{1}\right) \times O(1)\right)$ denotes the subgroup $\{(1,1),(1,-1)\}$ (resp. $\left.\left\{\left(I_{2 k_{1}}, 1\right),\left(I_{2 k_{1}},-1\right)\right\}\right)$. Now we may state the main theorem of this case.

THEOREM 5.1. Suppose a torus manifold $M$ has a codimension one extended $G$-action. If there is an exceptional orbit, then $(M, G)$ is essentially isomorphic to

$$
\left(\prod_{j=1}^{b} S^{2 m_{j}} \times_{\mathcal{A} \times \mathbb{Z}_{2}} N, \quad \prod_{j=1}^{b} S O\left(2 m_{j}+1\right) \times H\right),
$$

such that $(N, H)$ is one of the followings:

\begin{tabular}{|c|c|c|}
\hline & $N$ & $H$ \\
\hline \hline$(a)$ & $\left(\prod_{i=1}^{a} S^{2 l_{i}+1}\right) \times_{T^{a}} S\left(\mathbb{C}_{\mathfrak{c}}^{k_{1}} \oplus \mathbb{R}\right)$ & $\prod_{i=1}^{a} S U\left(l_{i}+1\right) \times U\left(k_{1}\right)$ \\
\hline$(b)$ & $\prod_{i=1}^{a} \mathbb{C} P\left(l_{i}\right) \times S\left(\mathbb{R}^{2 k_{1}} \oplus \mathbb{R}\right)$ & $\prod_{i=1}^{a} S U\left(l_{i}+1\right) \times S O\left(2 k_{1}\right)$ \\
\hline
\end{tabular}

where $\mathcal{A}$ acts on $\prod_{j=1}^{b} S^{2 m_{j}}$ as the subgroup $\prod_{j=1}^{b} \mathbb{Z}_{2}$ and on the fibre of $N$ through the following representations:

(a): $\sigma_{\mathbb{C}}: \mathcal{A} \rightarrow\{ \pm 1\} \subset S^{1}$ on $S\left(\mathbb{C}_{\mathfrak{c}}^{k_{1}} \oplus \mathbb{R}\right) \cap \mathbb{C}_{\mathfrak{c}}^{k_{1}} ;$

(b): $\sigma_{\mathbb{R}}: \mathcal{A} \rightarrow\left\{ \pm I_{2 k_{1}}\right\} \subset S O\left(2 k_{1}\right)$ on $S\left(\mathbb{R}^{2 k_{1}} \oplus \mathbb{R}\right) \cap \mathbb{R}^{2 k_{1}}$;

respectively, and $\mathbb{Z}_{2}$ (i.e., the second factor of $\mathcal{A} \times \mathbb{Z}_{2}$ ) acts on $\prod_{j=1}^{b} S^{2 m_{j}}$ through the representation $\rho: \mathbb{Z}_{2} \rightarrow \prod_{j=1}^{b} \mathbb{Z}_{2}$ which satisfies $\rho\left(\mathbb{Z}_{2}\right) \cap \mathcal{A}=\{1\}$ and on the fibre of $N$ through the following representations:

(a): $\sigma_{\mathbb{C} \oplus \mathbb{R}}: \mathbb{Z}_{2} \rightarrow\{ \pm 1\} \times O(1) \subset S^{1} \times O(1)$ on $\mathbb{C}_{\mathfrak{c}}^{k_{1}} \oplus \mathbb{R}$;

(b): $\sigma_{\mathbb{R} \oplus \mathbb{R}}: \mathbb{Z}_{2} \rightarrow\left\{ \pm I_{2 k_{1}}\right\} \times O(1) \subset S O\left(2 k_{1}\right) \times O(1)$ on $\mathbb{R}^{2 k_{1}} \oplus \mathbb{R}$;

respectively, such that $\sigma\left(=\sigma_{\mathbb{C} \oplus \mathbb{R}}, \sigma_{\mathbb{R} \oplus \mathbb{R}}\right)$ and $\rho$ satisfy one of the followings:

(i): $\rho$ is non-trivial, and $\sigma\left(\mathbb{Z}_{2}\right)=O(1)$ or $\sigma\left(\mathbb{Z}_{2}\right)=\Delta$;

(ii): $\rho$ is trivial, and $\sigma\left(\mathbb{Z}_{2}\right)=\Delta$.

Here, $G$-actions of (a), (b) are the same as Theorem 3.1 (a), (c), respectively.

REMARK 5.2. All manifolds appeared in Theorem 5.1 (a), (b) are $\mathbb{Z}_{2}$-quotient of manifolds appeared in Theorem 3.1 (a), (c), respectively. These $\mathbb{Z}_{2}$-actions are defined by $\rho \times \sigma$ in Theorem 5.1. Moreover, we remark that torus actions of manifolds in Theorem 3.1 (a), (c) have the next property: two singular orbits are same (diffeomorphic to $G / H$ ). By the $\mathbb{Z}_{2}$-quotient defined in Theorem 5.1, these two same singular orbits in Theorem 3.1 (a), (c) go to just one singular orbit $G / K_{1}$. Then $G / K_{1}$ can be regarded as $\mathbb{Z}_{2} \backslash G / H$ (if $\rho$ is non-trivial) or $G / H$ (if $\rho$ is trivial). 
In order to prove Theorem 5.1, we divide this case (2) into two cases which correspond with the case (a) and (b) for the type of $K_{1}$ (see Section 4). We call them the case (2)-(a) and (2)-(b), respectively, i.e., the case (2)-(a) is that $G / K_{2}$ is an exceptional orbit and $G / K_{1}$ satisfies the case (a), and the case (2)-(b) is that $G / K_{2}$ is an exceptional orbit and $G / K_{1}$ satisfies the case (b).

In Section 6 and 7, we study the case (2)-(a) and (2)-(b), respectively. Before we go to Section 6 , we give some technical remarks to study the case $(2)$.

5.2. Remarks of the case (2). We have already analysed $X_{1}$ and attaching map $f: \partial X_{1} \rightarrow \partial X_{2}$ in Section 4.1.1, 4.1.2 and 4.2; therefore, in order to construct a $G$-manifold $M=X_{1} \cup_{f} X_{2}$, we may only analyse $X_{2}=G \times_{K_{2}} D(\mathbb{R})$, where $D(\mathbb{R})=D^{1} \subset \mathbb{R}(1$-dimensional disk $)$.

Because $K_{2} / K \simeq S^{0}$ and $G / K_{2}$ is an exceptional orbit, we can easily show that the slice representation $\sigma_{2}: K_{2} \longrightarrow O(1)$ always satisfies the following properties:

- $\sigma_{2}$ is surjective;

- $\operatorname{ker} \sigma_{2}=K$.

Hence, a tubular neighborhood $X_{2}=G \times_{K_{2}} D(\mathbb{R})$ is only determined by the inclusion $K_{2} \subset G$. Therefore, we may only analyse the inclusion $K \subset K_{2} \subset N(K ; G)$, (see $(5.1))$ in the case $(2)$. So we first need to compute $N(K ; G)$. In the remainder of this section, we compute $N(K ; G)$ in the cases (2)-(a) and (2)-(b).

First we assume that $K$ is in the case (2)-(a) (see Section 4.1.1). Because of the definition of $K$ in the case (2)-(a), we have that $N(K ; G)$ is as follows:

$$
\begin{array}{r}
N(K ; G)=\prod_{j=1}^{b} S\left(O\left(2 m_{j}\right) \times O(1)\right) \times \prod_{i \in I} W_{i} \times \prod_{i^{\prime} \in I^{\prime}} S\left(U\left(l_{i^{\prime}}\right) \times U(1)\right) \\
\times S\left(U\left(k_{1}-1\right) \times U(1)\right) \times T^{1},
\end{array}
$$

where if $l_{i}=1$ and $\alpha_{i}=0$ then $i \in I$, otherwise $i^{\prime} \in I^{\prime}\left(I \cup I^{\prime}=\{1, \cdots, a\}\right)$, and $W_{i}=N(S(U(1) \times U(1)) ; S U(2))$ for $i \in I$. However, if $l_{i}=1$ and $\alpha_{i}=0$ then we can regard $S U(2)$ as $S O(3)$ up to essential isomorphism $(S U(2) \approx S O(3))$. Regarding $\{1, \cdots, b\} \cup I$ as $\{1, \cdots, b\}$ and $I^{\prime}$ as $\{1, \cdots, a\}$ again, we can write $N(K ; G)$ as follows:

$$
\begin{aligned}
N(K ; G) & =\prod_{j=1}^{b} S\left(O\left(2 m_{j}\right) \times O(1)\right) \\
& \times \prod_{i=1}^{a} S\left(U\left(l_{i}\right) \times U(1)\right) \times S\left(U\left(k_{1}-1\right) \times U(1)\right) \times T^{1} .
\end{aligned}
$$

Next we assume that $K$ is in the case (2)-(b) (see Section 4.1.2). By the similar argument of the case (2)-(a), we can regard $N(K ; G)$ in the case (2)-(b) as follows:

$$
\begin{aligned}
N(K ; G) & =\prod_{j=1}^{b} S\left(O\left(2 m_{j}\right) \times O(1)\right) \\
& \times \prod_{i=1}^{a} S\left(U\left(l_{i}\right) \times U(1)\right) \times S\left(O\left(2 k_{1}-1\right) \times O(1)\right) .
\end{aligned}
$$

In the next two sections, we will analyse $K \subset K_{2} \subset N(K ; G)$. 


\section{The case (2)-(a)}

In this section, we study the case (2)-(a). From Section 4.1.1, we have that

$$
\begin{aligned}
& G=\prod_{j=1}^{b} S O\left(2 m_{j}+1\right) \times \prod_{i=1}^{a} S U\left(l_{i}+1\right) \times S U\left(k_{1}\right) \times T^{1}, \\
& K_{1}=\mathcal{S}_{1} \times \prod_{i=1}^{a} S\left(U\left(l_{i}\right) \times U(1)\right) \times S U\left(k_{1}\right) \times T^{1}, \\
& K=\left\{\left(A,\left(t_{1}, \cdots, t_{a}\right),\left(\begin{array}{cc}
X & 0 \\
0 & x
\end{array}\right), t\right) \mid \sigma_{1}(A) t_{1}^{\alpha_{1}} \cdots t_{a}^{\alpha_{a}} x t^{\alpha}=1\right\} .
\end{aligned}
$$

The elements of $K$ are often denoted by $\left(A,\left(t_{1}, \cdots, t_{a}\right), x, t\right)$ simply.

In order to analyse the inclusion $K \subset K_{2} \subset N(K ; G)$ (see (5.2) for $N(K ; G)$ ), we first define $p_{1}$ and $p_{2}$ as the following two natural projections:

$$
\begin{aligned}
& p_{1}: N(K ; G) \longrightarrow \prod_{j=1}^{b} S\left(O\left(2 m_{j}\right) \times O(1)\right) ; \\
& p_{2}: N(K ; G) \longrightarrow \prod_{i=1}^{a} S\left(U\left(l_{i}\right) \times U(1)\right) \times S\left(U\left(k_{1}-1\right) \times U(1)\right) \times T^{1} .
\end{aligned}
$$

Then we can easily prove the following lemma.

Lemma 6.1. For $p_{1}, p_{2}$ and $K$, the following properties hold:

(1) the image of $K$ by $p_{1}$ satisfies $p_{1}(K)=\mathcal{S}_{1}$;

(2) if $\sigma_{1}\left(\mathcal{S}_{1}\right)=\{1\}$, then $K=\mathcal{S}_{1} \times p_{2}(K)$ and

$$
p_{2}(K)=\left\{\left(\left(t_{1}, \cdots, t_{a}\right), x, t\right) \mid t_{1}^{\alpha_{1}} \cdots t_{a}^{\alpha_{a}} x t^{\alpha}=1\right\}
$$

(3) if $\sigma_{1}\left(\mathcal{S}_{1}\right)=\{ \pm 1\}$, then $\left(\mathcal{S}_{1} \times p_{2}(K)\right) / K \simeq \mathbb{Z}_{2}$ and $p_{2}(K)=\left\{\left(\left(t_{1}, \cdots, t_{a}\right), x, t\right) \mid t_{1}^{\alpha_{1}} \cdots t_{a}^{\alpha_{a}} x t^{\alpha}= \pm 1\right\}$.

Proof. We define $\mathcal{R}_{1} \subset K$ as follows (also see Section 3.3):

$$
\mathcal{R}_{1}=\left\{\left(A, e,\left(\begin{array}{cc}
J & 0 \\
0 & \sigma_{1}(A)
\end{array}\right), 1\right) \mid A \in \mathcal{S}_{1}\right\}
$$

where $e \in \prod_{i=1}^{a} S\left(U\left(l_{i}\right) \times U(1)\right)$ is the identity element and $J \in U\left(k_{1}-1\right)$ such that $J=I_{k_{1}-1}\left(\right.$ if $\left.\sigma_{1}(A)=1\right)$ or $\operatorname{det} J=-1$ with $J^{2}=I_{k_{1}-1}\left(\right.$ if $\left.\sigma_{1}(A)=-1\right)$. Then $\mathcal{R}_{1} \simeq \mathcal{S}_{1}$. It follows that the first statement holds. The second and third statements are proved by the definition of $K$ and the first property $p_{1}(K)=\mathcal{S}_{1}$.

Let $\mathcal{S}_{2}$ be a subgroup of $\prod_{j=1}^{b} S\left(O\left(2 m_{j}\right) \times O(1)\right)$ such that $\mathcal{S}_{2} / \mathcal{S}_{1} \simeq \mathbb{Z}_{2}$. Because $K_{2} / K \simeq S^{0}$, for $p_{s}\left(K_{2}\right)$ and $p_{s}(K)(s=1,2)$, one of the following four cases occurs:

(i): $p_{1}\left(K_{2}\right)=\mathcal{S}_{2}$, and $p_{2}\left(K_{2}\right)=p_{2}(K)$;

(ii): $p_{1}\left(K_{2}\right)=p_{1}(K)=\mathcal{S}_{1}$, and $p_{2}\left(K_{2}\right) / p_{2}(K) \simeq \mathbb{Z}_{2}$;

(iii): $p_{1}\left(K_{2}\right)=p_{1}(K)=\mathcal{S}_{1}$, and $p_{2}\left(K_{2}\right)=p_{2}(K)$;

(iv): otherwise, i.e., $p_{1}\left(K_{2}\right)=\mathcal{S}_{2}$, and $p_{2}\left(K_{2}\right) / p_{2}(K) \simeq \mathbb{Z}_{2}$.

We call the above cases the case (2)-(a)-(i), (2)-(a)-(ii), (2)-(a)-(iii) and (2)-(a)-(iv), respectively. 
6.1. The case (2)-(a)-(i). Suppose the case (2)-(a)-(i) occurs, that is,

$$
\begin{aligned}
& p_{1}\left(K_{2}\right) / p_{1}(K)=\mathcal{S}_{2} / \mathcal{S}_{1} \simeq \mathbb{Z}_{2}, \quad \text { and } \\
& p_{2}\left(K_{2}\right)=p_{2}(K)=\left\{\left(\left(t_{1}, \cdots, t_{a}\right), x, t\right) \mid t_{1}^{\alpha_{1}} \cdots t_{a}^{\alpha_{a}} x t^{\alpha} \in \sigma_{1}\left(\mathcal{S}_{1}\right) \subset\{ \pm 1\}\right\} .
\end{aligned}
$$

Because $K \subset p_{1}(K) \times p_{2}(K)$ and $K_{2} \subset p_{1}\left(K_{2}\right) \times p_{2}\left(K_{2}\right)$, in this case we have that

$$
K \subset \mathcal{S}_{1} \times p_{2}(K), \quad K \subset K_{2} \subset \mathcal{S}_{2} \times p_{2}(K) .
$$

First we assume $\sigma_{1}\left(\mathcal{S}_{1}\right)=\{ \pm 1\}$, then $\left(\mathcal{S}_{1} \times p_{2}(K)\right) / K \simeq \mathbb{Z}_{2}$ by Lemma 6.1 (3). Therefore, $\#\left(\mathcal{S}_{2} \times p_{2}(K)\right) / K=4$ by $\mathcal{S}_{2} / \mathcal{S}_{1} \simeq \mathbb{Z}_{2}$ and $K \subset \mathcal{S}_{1} \times p_{2}(K) \subset \mathcal{S}_{2} \times p_{2}(K)$ (where \#F is the number of the finite group $F$ ). Hence, by making use of $K_{2} / K \simeq$ $S^{0}$ and (6.1), we also have

$$
\left(\mathcal{S}_{2} \times p_{2}(K)\right) / K_{2} \simeq \mathbb{Z}_{2} .
$$

Because $p_{1}\left(K_{2}\right)=\mathcal{S}_{2}, K \subset K_{2} \cap\left(\mathcal{S}_{1} \times p_{2}(K)\right) \subset K_{2}$ and $K_{2} / K \simeq S^{0}$, we also have

$$
K_{2} \cap\left(\mathcal{S}_{1} \times p_{2}(K)\right)=K \text {. }
$$

Now we define the representation $\rho: \mathcal{S}_{1} \times p_{2}(K) \rightarrow \mathbb{Z}_{2}$ as follows:

$$
\rho\left(A,\left(t_{1}, \cdots, t_{a}\right), x, t\right)=\sigma_{1}(A) t_{1}^{\alpha_{1}} \cdots t_{a}^{\alpha_{a}} x t^{\alpha} .
$$

By the definition of $K$, we have $\operatorname{ker} \rho=K$. Let $\widetilde{\rho}: \mathcal{S}_{2} \times p_{2}(K) \rightarrow \mathbb{Z}_{2}$ be a lift of this representation, i.e., the restricted representation $\left.\widetilde{\rho}\right|_{\mathcal{S}_{1} \times p_{2}(K)}$ coincides with $\rho$. Because $\mathcal{S}_{2} / \mathcal{S}_{1} \simeq \mathbb{Z}_{2}$ and $\left.\rho\right|_{p_{2}(K)}=\left.\widetilde{\rho}\right|_{p_{2}(K)}$, this lift $\widetilde{\rho}$ is only determined by a representation $\sigma_{2}: \mathcal{S}_{2} \rightarrow \mathbb{Z}_{2}$ such that $\left.\sigma_{2}\right|_{\mathcal{S}_{1}}=\sigma_{1}$. Hence, we have the following lemma.

Lemma 6.2. Let $\widetilde{\rho}: \mathcal{S}_{2} \times p_{2}(K) \rightarrow \mathbb{Z}_{2}$ be a lift of $\rho$. Then there is a representation $\sigma_{2}: \mathcal{S}_{2} \rightarrow \mathbb{Z}_{2}$ such that $\left.\sigma_{2}\right|_{\mathcal{S}_{1}}=\sigma_{1}$ and $\tilde{\rho}$ is denoted as follows:

$$
\widetilde{\rho}\left(B,\left(t_{1}, \cdots, t_{a}\right), x, t\right)=\sigma_{2}(B) t_{1}^{\alpha_{1}} \cdots t_{a}^{\alpha_{a}} x t^{\alpha} .
$$

On the other hand, by (6.2), there is the following sequence:

$$
K_{2} \stackrel{\widetilde{i}}{\longrightarrow} \mathcal{S}_{2} \times p_{2}(K) \stackrel{\widetilde{r}}{\longrightarrow} \mathbb{Z}_{2},
$$

where $\widetilde{i}$ is an inclusion and $\widetilde{r}$ is the surjective representation induced by the projective representation $\mathcal{S}_{2} \times p_{2}(K) \rightarrow\left(\mathcal{S}_{2} \times p_{2}(K)\right) / K_{2}$. Let $r$ be the restricted representation $\left.\widetilde{r}\right|_{\mathcal{S}_{1} \times p_{2}(K)}$. Using (6.3), we see that the representation $r$ is induced by the natural surjection $\mathcal{S}_{1} \times p_{2}(K) \rightarrow\left(\mathcal{S}_{1} \times p_{2}(K)\right) / K$, i.e., there is the following restricted sequence:

$$
K \stackrel{i}{\longrightarrow} \mathcal{S}_{1} \times p_{2}(K) \stackrel{r}{\longrightarrow} \mathbb{Z}_{2},
$$

where $i$ is the natural inclusion. By the definition of $K$, we have that $r$ can be identified with $\rho$. Therefore, by Lemma 6.2 , we have that $\widetilde{r}=\widetilde{\rho}$ for some $\sigma_{2}: \mathcal{S}_{2} \rightarrow$ $\mathbb{Z}_{2}$. In other wards, there is the representation $\sigma_{2}: \mathcal{S}_{2} \rightarrow \mathbb{Z}_{2}$ such that

$$
\begin{aligned}
& K_{2}=\operatorname{ker} \widetilde{\rho}(=\operatorname{ker} \widetilde{r}) \\
= & \left\{\left(B,\left(t_{1}, \cdots, t_{a}\right),\left(\begin{array}{cc}
X & 0 \\
0 & x
\end{array}\right), t\right) \mid \sigma_{2}(B) t_{1}^{\alpha_{1}} \cdots t_{a}^{\alpha_{a}} x t^{\alpha}=1\right\} .
\end{aligned}
$$

Next we assume $\sigma_{1}\left(\mathcal{S}_{1}\right)=\{1\}$, then $K=\mathcal{S}_{1} \times p_{2}(K)$ by Lemma $6.1(2)$. Hence, by using (6.1) and the assumption of the case (2)-(a)-(i), we have

$$
K_{2}=\mathcal{S}_{2} \times p_{2}(K) .
$$


We can regard this case as the case that $\sigma_{2}\left(\mathcal{S}_{2}\right)=\{1\}$ in Eq.(6.4).

Therefore, in the case (2)-(a)-(i), the inclusion $K_{2} \subset N(K ; G)$ is completely determined by the subgroup $\mathcal{S}_{2} \subset \prod_{j=1}^{b} S\left(O\left(2 m_{j}\right) \times O(1)\right)$ and the representation $\sigma_{2}: \mathcal{S}_{2} \rightarrow \mathbb{Z}_{2}$ such that $\left.\sigma_{2}\right|_{\mathcal{S}_{1}}=\sigma_{1}$. Hence, using (4.1) and Remark 4.2, we can easily check the following manifold corresponds with $M=X_{1} \cup X_{2}$ up to essential isomorphism (by computing orbit types of the natural $G=\prod_{j=1}^{b} S O\left(2 m_{j}+1\right) \times$ $\prod_{i=1}^{a} S U\left(l_{i}+1\right) \times U\left(k_{1}\right)$ action $)$ :

$$
M=\prod_{j=1}^{b} S^{2 m_{j}} \times_{\mathcal{A} \times \mathbb{Z}_{2}}\left(\prod_{i=1}^{a} S^{2 l_{i}+1} \times_{T^{a}} S\left(\mathbb{C}_{\mathfrak{c}}^{k_{1}} \oplus \mathbb{R}\right)\right),
$$

where $\mathcal{A}=\mathcal{A}_{1} \simeq \mathcal{S}_{1} / \prod_{j=1}^{b} S O\left(2 m_{j}\right)$ and the $\mathcal{A}$-quotient is defined by the following actions:

- on $\prod_{j=1}^{b} S^{2 m_{j}}$ as the subgroup of $\prod_{j=1}^{b} \mathbb{Z}_{2}$;

- on $\mathbb{C}_{\mathfrak{c}}^{k_{1}}$ through the representation $\sigma_{1}: \mathcal{A} \rightarrow \mathbb{Z}_{2}$ (this representation is induced by $\sigma_{1}: \mathcal{S}_{1} \rightarrow \mathbb{Z}_{2}$ );

- on $\mathbb{R}$ trivially,

and the $\mathbb{Z}_{2}$-quotient is defined by the following actions:

- on $\prod_{j=1}^{b} S^{2 m_{j}}$ by a non-trivial representation $\rho: \mathbb{Z}_{2} \rightarrow \prod_{j=1}^{b} \mathbb{Z}_{2}$ which satisfies $\rho\left(\mathbb{Z}_{2}\right) \cap \mathcal{A}=\{1\}$ (this corresponds with $p_{1}\left(K_{2}\right)=\mathcal{S}_{2}$ );

- on $\mathbb{C}_{\mathfrak{c}}^{k_{1}}$ by a representation $\sigma_{2}: \mathbb{Z}_{2} \rightarrow\{ \pm 1\}$, where if $\sigma_{1}$ is trivial then $\sigma_{2}$ is also trivial (this corresponds with that if $\sigma_{1}\left(\mathcal{S}_{1}\right)=\{1\}$ then $\sigma_{2}\left(\mathcal{S}_{2}\right)=\{1\}$ );

- on $\mathbb{R}$ by the natural representation (this corresponds with the existence of an exceptional orbit).

Remark that $\mathcal{A} \times \mathbb{Z}_{2}$ acts on $\prod_{j=1}^{b} S^{2 m_{j}}$ freely because $\rho$ is non-trivial and satisfies $\rho\left(\mathbb{Z}_{2}\right) \cap \mathcal{A}=\{1\}$; therefore, $M$ is a manifold, more precisely a fibre bundle over $\prod_{j=1}^{b} S^{2 m_{j}} /\left(\mathcal{A} \times \mathbb{Z}_{2}\right)$ with the fibre $\prod_{i=1}^{a} S^{2 l_{i}+1} \times_{T^{a}} S\left(\mathbb{C}_{\mathfrak{c}}^{k_{1}} \oplus \mathbb{R}\right)$.

6.2. The case (2)-(a)-(ii). Suppose the case (2)-(a)-(ii) occurs, that is,

$$
\begin{aligned}
& p_{1}\left(K_{2}\right)=p_{1}(K)=\mathcal{S}_{1} \quad \text { and } \\
& p_{2}\left(K_{2}\right) / p_{2}(K) \simeq \mathbb{Z}_{2} .
\end{aligned}
$$

First we prove the following lemma.

Lemma 6.3. Suppose that $p_{2}\left(K_{2}\right) / p_{2}(K) \simeq \mathbb{Z}_{2}$. Then the inclusion $p_{2}\left(K_{2}\right) \subset$ $p_{2}(N(K ; G))$ is unique. Furthermore, we have

$$
p_{2}\left(K_{2}\right)=\left\{\left(\left(t_{1}, \cdots, t_{a}\right),\left(\begin{array}{cc}
X & 0 \\
0 & x
\end{array}\right), t\right) \mid t_{1}^{\alpha_{1}} \cdots t_{a}^{\alpha_{a}} x t^{\alpha} \in \mathbb{Z}_{m}\right\},
$$

where $m=2$ if $\sigma_{1}\left(\mathcal{S}_{1}\right)=\{1\}$ or $m=4$ if $\sigma_{1}\left(\mathcal{S}_{1}\right)=\{ \pm 1\}$.

Proof. We first remark that $\sigma_{1}\left(\mathcal{S}_{1}\right) \subset\{ \pm 1\}$, by Section 4.1.1.

Consider the following surjective representation $\sigma: p_{2}(N(K ; G))=\prod_{i=1}^{a} S\left(U\left(l_{i}\right) \times\right.$ $U(1)) \times S\left(U\left(k_{1}-1\right) \times U(1)\right) \times T^{1} \rightarrow S^{1}$ (induced by the slice representation $\sigma_{1}$ ): $\sigma\left(\left(t_{1}, \cdots, t_{a}\right),\left(\begin{array}{cc}X & 0 \\ 0 & x\end{array}\right), t\right)=t_{1}^{\alpha_{1}} \cdots t_{a}^{\alpha_{a}} x t^{\alpha} \quad\left(\right.$ or $\quad t_{1}^{\alpha_{1}} \cdots t_{a}^{\alpha_{a}} t \quad$ for $\left.\quad k_{1}=1\right)$.

Then we see that $p_{2}(K)=\sigma^{-1}\left(\sigma_{1}\left(\mathcal{S}_{1}\right)\right) \subset p_{2}\left(K_{2}\right)$ by the definition of $K$. Therefore, we have that $\sigma\left(p_{2}(K)\right)=\sigma_{1}\left(\mathcal{S}_{1}\right) \subset \sigma\left(p_{2}\left(K_{2}\right)\right)$. 
Because $p_{2}\left(K_{2}\right) / p_{2}(K) \simeq \mathbb{Z}_{2}$, we also have that

$$
\sigma\left(p_{2}\left(K_{2}\right)\right) / \sigma\left(p_{2}(K)\right) \subset \mathbb{Z}_{2}\left(\subset \sigma\left(p_{2}(N(K ; G))\right) / \sigma\left(p_{2}(K)\right) \simeq S^{1}\right) .
$$

If $\sigma\left(p_{2}\left(K_{2}\right)\right) / \sigma\left(p_{2}(K)\right)=\{1\} \subset \mathbb{Z}_{2}$, then $\sigma\left(p_{2}\left(K_{2}\right)\right)=\sigma\left(p_{2}(K)\right)=\sigma_{1}\left(\mathcal{S}_{1}\right)$. Hence, we have $p_{2}\left(K_{2}\right) \subset \sigma^{-1}\left(\sigma_{1}\left(\mathcal{S}_{1}\right)\right)=p_{2}(K)$. However, this gives a contradiction, because $p_{2}\left(K_{2}\right) / p_{2}(K) \simeq \mathbb{Z}_{2}$. Therefore, we have

$$
\sigma\left(p_{2}\left(K_{2}\right)\right) / \sigma\left(p_{2}(K)\right)=\mathbb{Z}_{2} \subset S^{1} .
$$

It follows that there are the following two cases:

- if $\sigma_{1}\left(\mathcal{S}_{1}\right)=\sigma\left(p_{2}(K)\right)=\{1\}$, then $\sigma\left(p_{2}\left(K_{2}\right)\right)=\mathbb{Z}_{2}=\{ \pm 1\} \subset S^{1}$; hence, $p_{2}\left(K_{2}\right) \subset \sigma^{-1}\left(\mathbb{Z}_{2}\right)$;

- if $\sigma_{1}\left(\mathcal{S}_{1}\right)=\sigma\left(p_{2}(K)\right)=\{ \pm 1\}$, then $\sigma\left(p_{2}\left(K_{2}\right)\right)=\mathbb{Z}_{4}=\{ \pm 1, \pm i\} \subset S^{1}$; hence, $p_{2}\left(K_{2}\right) \subset \sigma^{-1}\left(\mathbb{Z}_{4}\right)$.

Because $p_{2}\left(K_{2}\right) / p_{2}(K) \simeq \mathbb{Z}_{2}$ and $p_{2}(K)=\sigma^{-1}\left(\sigma_{1}\left(\mathcal{S}_{1}\right)\right)$, we can easily show that $p_{2}\left(K_{2}\right)=\sigma^{-1}\left(\mathbb{Z}_{2}\right)$ for the case $\sigma_{1}\left(\mathcal{S}_{1}\right)=\{1\}$ and $p_{2}\left(K_{2}\right)=\sigma^{-1}\left(\mathbb{Z}_{4}\right)$ for the case $\sigma_{1}\left(\mathcal{S}_{1}\right)=\{ \pm 1\}$. Hence, we have that the inclusion of $p_{2}\left(K_{2}\right) \subset p_{2}(N(K ; G))$ is uniquely determined by $\sigma^{-1}\left(\mathbb{Z}_{2}\right)$ or $\sigma^{-1}\left(\mathbb{Z}_{4}\right)$. Thus, we have the statement of this lemma.

Assume $\sigma_{1}\left(\mathcal{S}_{1}\right)=\{ \pm 1\}$. Then $m=4$ by Lemma 6.3. Let $\widetilde{\rho}: \mathcal{S}_{1} \times p_{2}\left(K_{2}\right) \rightarrow \mathbb{Z}_{4}$ be the following representation:

$$
\widetilde{\rho}\left(A,\left(t_{1}, \cdots, t_{a}\right), x, t\right)=\sigma_{1}(A) t_{1}^{\alpha_{1}} \cdots t_{a}^{\alpha_{a}} x t^{\alpha} \in \mathbb{Z}_{4} .
$$

We can easily show that $\widetilde{\rho}^{-1}\left(\mathbb{Z}_{2}\right)=\mathcal{S}_{1} \times p_{2}(K)$ and ker $\widetilde{\rho}=K$. Because $K_{2} / K \simeq S^{0}$, we also have $\widetilde{\rho}\left(K_{2}\right)=\mathbb{Z}_{2} \subset \mathbb{Z}_{4}$. Hence, we have $K_{2} \subset \widetilde{\rho}^{-1}\left(\mathbb{Z}_{2}\right)=\mathcal{S}_{1} \times p_{2}(K)$. However, this gives a contradiction, because $p_{2}\left(K_{2}\right) / p_{2}(K) \simeq \mathbb{Z}_{2}$. Therefore, we have $\sigma_{1}\left(\mathcal{S}_{1}\right)=\{1\}$.

Thus, we have $K=\mathcal{S}_{1} \times p_{2}(K)$ by Lemma $6.1(2)$. Because $K_{2} / K \simeq S^{0} \simeq$ $\mathbb{Z}_{2} \simeq p_{2}\left(K_{2}\right) / p_{2}(K)$ and $K \subset K_{2} \subset \mathcal{S}_{1} \times p_{2}\left(K_{2}\right)$, the subgroup $K_{2} \subset N(K ; G)$ in the case (2)-(a)-(ii) is as follows:

$$
K_{2}=\mathcal{S}_{1} \times p_{2}\left(K_{2}\right),
$$

where $p_{2}\left(K_{2}\right)$ is the group in Lemma 6.3 with $m=2$. Hence, with a method similar to that demonstrated in the case (2)-(a)-(i) (Section 6.1), we can easily check the following manifold corresponds with $M=X_{1} \cup X_{2}$ up to essential isomorphism:

$$
M=\prod_{j=1}^{b} S^{2 m_{j}} \times_{\mathcal{A} \times \mathbb{Z}_{2}}\left(\prod_{i=1}^{a} S^{2 l_{i}+1} \times_{T^{a}} S\left(\mathbb{C}_{\mathfrak{c}}^{k_{1}} \oplus \mathbb{R}\right)\right),
$$

where the $\mathcal{A}\left(=\mathcal{A}_{1}\right)$-quotient is defined by the following actions:

- on $\prod_{j=1}^{b} S^{2 m_{j}}$ as the subgroup of $\prod_{j=1}^{b} \mathbb{Z}_{2}$;

- on $\mathbb{C}_{\mathfrak{c}}^{k_{1}}$ trivially (this corresponds with that $\sigma_{1}\left(\mathcal{S}_{1}\right)=\{1\}$ );

- on $\mathbb{R}$ trivially

and the $\mathbb{Z}_{2}$-quotient is defined by the following actions:

- on $\prod_{j=1}^{b} S^{2 m_{j}}$ trivially (this corresponds with $p_{1}\left(K_{2}\right)=\mathcal{S}_{1}$ );

- on $\mathbb{C}_{\mathfrak{c}}^{k_{1}}$ by a non-trivial representation $\sigma_{2}: \mathbb{Z}_{2} \rightarrow\{ \pm 1\}$ (this corresponds with $\left.p_{2}\left(K_{2}\right) / p_{2}(K) \simeq \mathbb{Z}_{2}\right)$;

- on $\mathbb{R}$ by the natural representation. 
Remark that $M$ is also $G$-equivariantly diffeomorphic to the following manifold $\left(\right.$ where $\left.G=\prod_{j=1}^{b} S O\left(2 m_{j}+1\right) \times \prod_{i=1}^{a} S U\left(l_{i}+1\right) \times U\left(k_{1}\right)\right)$ :

$$
\prod_{j=1}^{b} S^{2 m_{j}} / \mathcal{A} \times\left(\prod_{i=1}^{a} S^{2 l_{i}+1} \times_{T^{a}} P\left(\mathbb{C}_{\mathfrak{c}}^{k_{1}} \oplus \mathbb{R}\right)\right)
$$

where $P\left(\mathbb{C}_{\mathfrak{c}}^{k_{1}} \oplus \mathbb{R}\right)$ is the real projective space.

6.3. The case (2)-(a)-(iii). Suppose the case (2)-(a)-(iii) occurs, that is,

$$
\begin{aligned}
& p_{1}\left(K_{2}\right)=p_{1}(K)=\mathcal{S}_{1} \quad \text { and } \\
& p_{2}\left(K_{2}\right)=p_{2}(K) .
\end{aligned}
$$

For this case (iii), the following lemma holds.

LEMma 6.4. Suppose that the case (iii) occurs. Then $\sigma_{1}\left(\mathcal{S}_{1}\right)=\{ \pm 1\}$, and $p_{2}\left(K_{2}\right) \subset p_{2}(N(K ; G))$ is

$p_{2}\left(K_{2}\right)=p_{2}(K)=\left\{\left(\left(t_{1}, \cdots, t_{a}\right),\left(\begin{array}{cc}X & 0 \\ 0 & x\end{array}\right), t\right) \mid t_{1}^{\alpha_{1}} \cdots t_{a}^{\alpha_{a}} x t^{\alpha}= \pm 1\right\}$.

Furthermore, we have $K_{2}=\mathcal{S}_{1} \times p_{2}(K)$.

Proof. If $\sigma_{1}\left(\mathcal{S}_{1}\right)=\{1\}$, then we have $K=\mathcal{S}_{1} \times p_{2}(K)$ by Lemma 6.1 (2). It follows that $K=p_{1}\left(K_{2}\right) \times p_{2}\left(K_{2}\right) \supset K_{2}$ by the assumption of the case (iii). This gives a contradiction to $K_{2} / K \simeq S^{0}$. Hence, we have $\sigma_{1}\left(\mathcal{S}_{1}\right)=\{ \pm 1\}$. Moreover, we have that

$$
p_{2}\left(K_{2}\right)=p_{2}(K)=\left\{\left(\left(t_{1}, \cdots, t_{a}\right),\left(\begin{array}{cc}
X & 0 \\
0 & x
\end{array}\right), t\right) \mid t_{1}^{\alpha_{1}} \cdots t_{a}^{\alpha_{a}} x t^{\alpha}= \pm 1\right\}
$$

by Lemma $6.1(3)$.

By the definition of $p_{1}, p_{2}$ and the assumptions of the case (iii), we have the following relation:

$$
K \subset K_{2} \subset \mathcal{S}_{1} \times p_{2}(K)
$$

By the definition of $K$, we can easily check that $\left(\mathcal{S}_{1} \times p_{2}(K)\right) / K \simeq \mathbb{Z}_{2}$. Therefore, we have $K_{2}=\mathcal{S}_{1} \times p_{2}(K)$.

By using Lemma 6.4 and a method similar to that demonstrated in the previous cases, we can easily check that the following manifold corresponds with $M=X_{1} \cup X_{2}$ up to essential isomorphism:

$$
M=\prod_{j=1}^{b} S^{2 m_{j}} \times_{\mathcal{A} \times \mathbb{Z}_{2}}\left(\prod_{i=1}^{a} S^{2 l_{i}+1} \times_{T^{a}} S\left(\mathbb{C}_{\mathfrak{c}}^{k_{1}} \oplus \mathbb{R}\right)\right)
$$

where the $\mathcal{A}\left(=\mathcal{A}_{1}\right)$-quotient is defined by the following actions:

- on $\prod_{j=1}^{b} S^{2 m_{j}}$ as the subgroup of $\prod_{j=1}^{b} \mathbb{Z}_{2}$;

- on $\mathbb{C}_{\mathfrak{c}}^{k_{1}}$ through the non-trivial representation $\sigma_{1}: \mathcal{A} \rightarrow \mathbb{Z}_{2}$ (this corresponds with $\left.\sigma_{1}\left(\mathcal{S}_{1}\right)=\{ \pm 1\}\right)$;

- on $\mathbb{R}$ trivially,

and the $\mathbb{Z}_{2}$-quotient is defined by the following actions:

- on $\prod_{j=1}^{b} S^{2 m_{j}}$ trivially (this corresponds with $p_{1}\left(K_{2}\right)=\mathcal{S}_{1}$ ); 
- on $\mathbb{C}_{\mathfrak{c}}^{k_{1}}$ by a non-trivial representation $\sigma_{2}: \mathbb{Z}_{2} \rightarrow\{ \pm 1\}$ (this is known by using $\sigma_{1}\left(\mathcal{S}_{1}\right)=\{ \pm 1\}$ and $\left.p_{2}\left(K_{2}\right)=p_{2}(K)\right)$;

- on $\mathbb{R}$ by the natural representation.

Remark that $M$ is also $G$-equivariantly diffeomorphic to the following manifold as well as the case (2)-(a)-(ii):

$$
\prod_{j=1}^{b} S^{2 m_{j}} \times_{\mathcal{A}}\left(\prod_{i=1}^{a} S^{2 l_{i}+1} \times_{T^{a}} P\left(\mathbb{C}_{\mathfrak{c}}^{k_{1}} \oplus \mathbb{R}\right)\right) .
$$

6.4. The case (2)-(a)-(iv). Suppose the case (2)-(a)-(iv) occurs, that is,

$$
\begin{aligned}
& p_{1}\left(K_{2}\right) / p_{1}(K)=\mathcal{S}_{2} / \mathcal{S}_{1} \simeq \mathbb{Z}_{2} \quad \text { and } \\
& p_{2}\left(K_{2}\right) / p_{2}(K) \simeq \mathbb{Z}_{2} .
\end{aligned}
$$

First, we remark that Lemma 6.3 can be used in this case because of $p_{2}\left(K_{2}\right) / p_{2}(K) \simeq$ $\mathbb{Z}_{2}$.

Assume $\sigma_{1}\left(\mathcal{S}_{1}\right)=\{ \pm 1\}$. Then we have $m=4$ for the group $p_{2}\left(K_{2}\right)$ in Lemma 6.3. Let $\widetilde{\rho}: \mathcal{S}_{2} \times p_{2}\left(K_{2}\right) \rightarrow \mathbb{Z}_{4}$ be the following representation:

$$
\widetilde{\rho}\left(B,\left(t_{1}, \cdots, t_{a}\right), x, t\right)=\sigma_{2}(B) t_{1}^{\alpha_{1}} \cdots t_{a}^{\alpha_{a}} x t^{\alpha} \in \mathbb{Z}_{4},
$$

where $\sigma_{2}: \mathcal{S}_{2} \rightarrow \mathbb{Z}_{4}$ is some representation such that $\left.\sigma_{2}\right|_{\mathcal{S}_{1}}=\sigma_{1}$. Then we can easily show that $\sigma_{2}: \mathcal{S}_{2} \rightarrow \mathbb{Z}_{2}$, i.e., the image of $\mathcal{S}_{2}$ is in $\mathbb{Z}_{2} \subset \mathbb{Z}_{4}$, because $\prod_{j=1}^{b} S O\left(2 m_{j}\right) \subset \operatorname{ker} \sigma_{1} \subset \operatorname{ker} \sigma_{2}$ and $\mathcal{S}_{2} / \prod_{j=1}^{b} S O\left(2 m_{j}\right) \subset \prod_{j=1}^{b} \mathbb{Z}_{2}$. Hence, we have that

$$
\operatorname{ker} \widetilde{\rho} \subset \mathcal{S}_{2} \times p_{2}(K) \text {. }
$$

Consider the restricted representation $\left.\widetilde{\rho}\right|_{K_{2}}: K_{2} \rightarrow \mathbb{Z}_{4}$. Then we have the following sequence:

$$
\left.K \subset \operatorname{ker} \widetilde{\rho}\right|_{K_{2}}=\operatorname{ker} \widetilde{\rho} \cap K_{2} \subset K_{2}
$$

because of the definitions of $K$ and $\sigma_{2}$. Therefore, by $K_{2} / K \simeq S^{0}$, we have that $\left.\operatorname{ker} \widetilde{\rho}\right|_{K_{2}}=K_{2}$ or $K$. If ker $\left.\widetilde{\rho}\right|_{K_{2}}=K_{2}$, then we have $p_{2}\left(K_{2}\right)=p_{2}(K)$ by (6.5). This gives a contradiction to $p_{2}\left(K_{2}\right) / p_{2}(K)=\mathbb{Z}_{2}$. Hence, we have ker $\left.\widetilde{\rho}\right|_{K_{2}}=K$. Then we have

$$
K_{2} / K=K_{2} /\left.\operatorname{ker} \widetilde{\rho}\right|_{K_{2}} \simeq \widetilde{\rho}\left(K_{2}\right)=\mathbb{Z}_{2}=\{ \pm 1\} \subset \mathbb{Z}_{4} .
$$

It also follows that $K_{2} \subset \widetilde{\rho}^{-1}\left(\mathbb{Z}_{2}\right)=\mathcal{S}_{2} \times p_{2}(K)$; therefore, $p_{2}\left(K_{2}\right)=p_{2}(K)$. This also gives a contradiction to $p_{2}\left(K_{2}\right) / p_{2}(K)=\mathbb{Z}_{2}$. Thus, we have $\sigma_{1}\left(\mathcal{S}_{1}\right)=\{1\}$. Hence, by Lemma $6.1(2)$, we have $K=\mathcal{S}_{1} \times p_{2}(K)$.

Because $K_{2} / K=K_{2} /\left(\mathcal{S}_{1} \times p_{2}(K)\right) \simeq \mathbb{Z}_{2}$ and $\left(\mathcal{S}_{2} \times p_{2}\left(K_{2}\right)\right) / K \simeq \mathbb{Z}_{2} \times \mathbb{Z}_{2}$ (the assumption of (2)-(a)-(iv)), we have that

$$
\left(\mathcal{S}_{2} \times p_{2}\left(K_{2}\right)\right) / K_{2} \simeq \mathbb{Z}_{2} .
$$

Again, we define $\widetilde{\rho}: \mathcal{S}_{2} \times p_{2}\left(K_{2}\right) \rightarrow \mathbb{Z}_{2} \times \mathbb{Z}_{2}$ as follows:

$$
\widetilde{\rho}\left(B,\left(t_{1}, \cdots, t_{a}\right), x, t\right)=\left(\sigma_{2}(B), t_{1}^{\alpha_{1}} \cdots t_{a}^{\alpha_{a}} x t^{\alpha}\right) \in \mathbb{Z}_{2} \times \mathbb{Z}_{2},
$$

where $\sigma_{2}: \mathcal{S}_{2} \rightarrow \mathbb{Z}_{2}$ such that $\left.\sigma_{2}\right|_{\mathcal{S}_{1}}=\sigma_{1}$. Then we have ker $\widetilde{\rho}=K$ by $K=$ $\mathcal{S}_{1} \times p_{2}(K)$ and $\sigma_{1}\left(\mathcal{S}_{1}\right)=\{1\}$. Because $K_{2} / K \simeq \mathbb{Z}_{2}$, we have that $\widetilde{\rho}\left(K_{2}\right) \simeq \mathbb{Z}_{2}$, i.e., $\widetilde{\rho}\left(K_{2}\right)=\mathbb{Z}_{2} \times\{1\},\{1\} \times \mathbb{Z}_{2}$ or $\Delta=\{(1,1),(-1,-1)\}$. If $\widetilde{\rho}\left(K_{2}\right)=\mathbb{Z}_{2} \times\{1\}$ or $\{1\} \times \mathbb{Z}_{2}$, then this gives a contradiction to that $p_{2}\left(K_{2}\right) / p_{2}(K)=\mathbb{Z}_{2}$ or $p_{1}\left(K_{2}\right)=\mathcal{S}_{1}$, 
respectively. Therefore, we have that $\widetilde{\rho}\left(K_{2}\right)=\Delta$. Moreover, by using (6.6), the subgroup $K_{2} \subset \mathcal{S}_{2} \times p_{2}\left(K_{2}\right)$ can be denoted as follows:

$$
\tilde{\rho}^{-1}(\Delta)=K_{2}=\left\{\left(B, t_{1}, \cdots, t_{a}, x, t\right) \mid \sigma_{2}(B) t_{1}^{\alpha_{a}} \cdots t_{a}^{\alpha_{a}} x t^{\alpha}=1\right\},
$$

where $\sigma_{2}: \mathcal{S}_{2} \rightarrow \mathbb{Z}_{2}$ such that $\operatorname{ker} \sigma_{2}=\mathcal{S}_{1}$. Hence $K_{2} \subset N(K ; G)$ is completely determined by $\mathcal{S}_{2}$ and $\sigma_{2}$ in the case (2)-(a)-(iv).

With a method similar to that demonstrated in the previous cases, we can easily check the following manifold corresponds with $M=X_{1} \cup X_{2}$ up to essential isomorphism:

$$
M=\prod_{j=1}^{b} S^{2 m_{j}} \times_{\mathcal{A} \times \mathbb{Z}_{2}}\left(\prod_{i=1}^{a} S^{2 l_{i}+1} \times_{T^{a}} S\left(\mathbb{C}_{\mathfrak{c}}^{k_{1}} \oplus \mathbb{R}\right)\right),
$$

where the $\mathcal{A}\left(=\mathcal{A}_{1}\right)$-quotient is defined by the following actions:

- on $\prod_{j=1}^{b} S^{2 m_{j}}$ as the subgroup of $\prod_{j=1}^{b} \mathbb{Z}_{2}$;

- on $\mathbb{C}_{\mathfrak{c}}^{k_{1}}$ trivially (this corresponds with $\sigma_{1}\left(\mathcal{S}_{1}\right)=\{1\}$ );

- on $\mathbb{R}$ trivially,

and the $\mathbb{Z}_{2}$-quotient is defined by the following actions:

- on $\prod_{j=1}^{b} S^{2 m_{j}}$ by a non-trivial representation $\rho: \mathbb{Z}_{2} \rightarrow \prod_{j=1}^{b} \mathbb{Z}_{2}$ which satisfies $\rho\left(\mathbb{Z}_{2}\right) \cap \mathcal{A}=\{1\}$ (this corresponds with $p_{1}\left(K_{2}\right)=\mathcal{S}_{2}$ );

- on $\mathbb{C}_{\mathfrak{c}}^{k_{1}}$ by a non-trivial representation $\sigma_{2}: \mathbb{Z}_{2} \rightarrow\{ \pm 1\}$ (this corresponds with $\left.p_{2}\left(K_{2}\right) / p_{2}(K) \simeq \mathbb{Z}_{2}\right)$

- on $\mathbb{R}$ by the natural representation.

Remark that $M$ is also $G$-equivariantly diffeomorphic to the following manifold as well as the previous cases:

$$
\prod_{j=1}^{b} S^{2 m_{j}} / \mathcal{A} \times_{\mathbb{Z}_{2}}\left(\prod_{i=1}^{a} S^{2 l_{i}+1} \times_{T^{a}} S\left(\mathbb{C}_{\mathfrak{c}}^{k_{1}} \oplus \mathbb{R}\right)\right) .
$$

6.5. Summary of the results from (2)-(a)-(i) to (iv). In summary, we can state the result of the case (2)-(a) as follows. Let $(M, G)$ be the pair in the case (2)-(a). Then $(M, G)$ is essentially isomorphism to the followings:

$$
\begin{aligned}
& M=\prod_{j=1}^{b} S^{2 m_{j}} \times_{\mathcal{A} \times \mathbb{Z}_{2}}\left(\prod_{i=1}^{a} S^{2 l_{i}+1} \times_{T^{a}} S\left(\mathbb{C}_{\mathfrak{a}}^{k_{1}} \oplus \mathbb{R}\right)\right), \\
& G=\prod_{j=1}^{b} S O\left(2 m_{j}+1\right) \times \prod_{i=1}^{a} S U\left(l_{i}+1\right) \times U\left(k_{1}\right),
\end{aligned}
$$

where $G$ acts on $M$ naturally, and the $\mathcal{A}\left(=\mathcal{A}_{1}\right)$-quotient is defined by the following actions:

- on $\prod_{j=1}^{b} S^{2 m_{j}}$ as the subgroup of $\prod_{j=1}^{b} \mathbb{Z}_{2}$;

- on $\mathbb{C}_{\mathfrak{c}}^{k_{1}}$ through the representation $\sigma_{1}\left(=\sigma_{\mathbb{C}}\right): \mathcal{A} \rightarrow \mathbb{Z}_{2}$;

- on $\mathbb{R}$ trivially,

and the $\mathbb{Z}_{2}$-quotient is defined by the following actions:

- on $\prod_{j=1}^{b} S^{2 m_{j}}$ by a representation $\rho: \mathbb{Z}_{2} \rightarrow \prod_{j=1}^{b} \mathbb{Z}_{2}$ which satisfies $\rho\left(\mathbb{Z}_{2}\right) \cap \mathcal{A}=\{1\}$

- on $\mathbb{C}_{\mathfrak{c}}^{k_{1}}$ by a representation $\sigma_{2}: \mathbb{Z}_{2} \rightarrow\{ \pm 1\}$; 
- on $\mathbb{R}$ by the natural representation $\kappa: \mathbb{Z}_{2} \rightarrow O(1)$,

where $\rho$ or $\sigma_{2}$ is always non-trivial, i.e., the case that both of two representations $\rho$ and $\sigma_{2}$ are trivial does not occur. This corresponds with the first manifold in Theorem 5.1, where $\sigma_{2} \oplus \kappa=\sigma_{\mathbb{C} \oplus \mathbb{R}}$.

\section{The case (2)-(b)}

In this section, we study the case (2)-(b). From Section 4.1.2, we have that

$$
\begin{aligned}
G & =\prod_{j=1}^{b} S O\left(2 m_{j}+1\right) \times \prod_{i=1}^{a} S U\left(l_{i}+1\right) \times S O\left(2 k_{1}\right), \\
K_{1} & =\mathcal{S}_{1} \times \prod_{i=1}^{a} S\left(U\left(l_{i}\right) \times U(1)\right) \times S O\left(2 k_{1}\right), \\
K & =\prod_{i=1}^{a} S\left(U\left(l_{i}\right) \times U(1)\right) \\
& \times\left\{\left(A,\left(\begin{array}{cc}
X & 0 \\
0 & x
\end{array}\right)\right) \in \mathcal{S}_{1} \times S\left(O\left(2 k_{1}-1\right) \times O(1)\right) \mid \sigma_{1}(A) x=1\right\} \\
& =\prod_{i=1}^{a} S\left(U\left(l_{i}\right) \times U(1)\right) \times K^{\prime} .
\end{aligned}
$$

An element in $\mathcal{S}_{1} \times S\left(O\left(2 k_{1}-1\right) \times O(1)\right)$ is often denoted by $(A, x)$. In this case, by Section 5.2 , we may only analyze the inclusion of $K_{2}$ such that $K \subset K_{2} \subset N(K ; G)$ and $N(K ; G)$ is known as $(5.3)$.

Because $K \subset K_{2} \subset N(K ; G)$ and $K_{2} / K \simeq S^{0}$, we have that

$$
K_{2}=\prod_{i=1}^{a} S\left(U\left(l_{i}\right) \times U(1)\right) \times K_{2}^{\prime},
$$

where $K_{2}^{\prime} \subset \prod_{j=1}^{b} S\left(O\left(2 m_{j}\right) \times O(1)\right) \times S\left(O\left(2 k_{1}-1\right) \times O(1)\right)$ such that $K_{2}^{\prime} / K^{\prime} \simeq \mathbb{Z}_{2}$.

Let $p_{1}$ and $p_{2}$ be the following two natural projections:

$$
\begin{aligned}
& p_{1}: \prod_{j=1}^{b} S\left(O\left(2 m_{j}\right) \times O(1)\right) \times S\left(O\left(2 k_{1}-1\right) \times O(1)\right) \longrightarrow \prod_{j=1}^{b} S\left(O\left(2 m_{j}\right) \times O(1)\right) ; \\
& p_{2}: \prod_{j=1}^{b} S\left(O\left(2 m_{j}\right) \times O(1)\right) \times S\left(O\left(2 k_{1}-1\right) \times O(1)\right) \longrightarrow S\left(O\left(2 k_{1}-1\right) \times O(1)\right) .
\end{aligned}
$$

Similarly to Lemma 6.1 , we can easily prove the following lemma.

LEMMA 7.1. For $p_{1}, p_{2}$ and $K^{\prime}$, the following properties hold:

(1) the image of $K^{\prime}$ by $p_{1}$ satisfies $p_{1}\left(K^{\prime}\right)=\mathcal{S}_{1}$;

(2) if $\sigma_{1}\left(\mathcal{S}_{1}\right)=\{1\}$, then $p_{2}\left(K^{\prime}\right)=S O\left(2 k_{1}-1\right)$ and $K^{\prime}=\mathcal{S}_{1} \times S O\left(2 k_{1}-1\right)$;

(3) if $\sigma_{1}\left(\mathcal{S}_{1}\right)=\{ \pm 1\}$, then $p_{2}\left(K^{\prime}\right)=S\left(O\left(2 k_{1}-1\right) \times O(1)\right)$ and $\left(\mathcal{S}_{1} \times S\left(O\left(2 k_{1}-\right.\right.\right.$ 1) $\times O(1))) / K^{\prime} \simeq \mathbb{Z}_{2}$.

PROOF. The first statement is proved by making use of the subgroup $\mathcal{R}_{1}$ in Section 3.5. The second and third statements are proved by the definition of $K^{\prime}$ and the first property $p_{1}\left(K^{\prime}\right)=\mathcal{S}_{1}$. 
Because $K_{2}^{\prime} / K^{\prime} \simeq \mathbb{Z}_{2}$, similarly to the case (2)-(a), one of the following four cases occurs:

(i): $p_{1}\left(K_{2}^{\prime}\right)=\mathcal{S}_{2}$, and $p_{2}\left(K_{2}^{\prime}\right)=p_{2}\left(K^{\prime}\right)$;

(ii): $p_{1}\left(K_{2}^{\prime}\right)=p_{1}\left(K^{\prime}\right)=\mathcal{S}_{1}$, and $p_{2}\left(K_{2}^{\prime}\right) / p_{2}\left(K^{\prime}\right) \simeq \mathbb{Z}_{2}$;

(iii): $p_{1}\left(K_{2}^{\prime}\right)=p_{1}\left(K^{\prime}\right)=\mathcal{S}_{1}$, and $p_{2}\left(K_{2}^{\prime}\right)=p_{2}\left(K^{\prime}\right)$;

(iv): otherwise, i.e., $p_{1}\left(K_{2}^{\prime}\right)=\mathcal{S}_{2}$, and $p_{2}\left(K_{2}^{\prime}\right) / p_{2}\left(K^{\prime}\right) \simeq \mathbb{Z}_{2}$,

where $\mathcal{S}_{2}$ is a subgroup of $\prod_{j=1}^{b} S\left(O\left(2 m_{j}\right) \times O(1)\right)$ such that $\mathcal{S}_{2} / \mathcal{S}_{1} \simeq \mathbb{Z}_{2}$. We call the above cases the case (2)-(b)-(i), (2)-(b)-(ii), (2)-(b)-(iii) and (2)-(b)-(iv), respectively.

7.1. The case (2)-(b)-(i). Suppose the case (2)-(b)-(i) occurs, that is,

$$
p_{1}\left(K_{2}^{\prime}\right)=\mathcal{S}_{2} \quad \text { and } \quad p_{2}\left(K_{2}^{\prime}\right)=p_{2}\left(K^{\prime}\right) .
$$

If $\sigma_{1}\left(\mathcal{S}_{1}\right)=\{ \pm 1\}$, then $K^{\prime}$ is defined as $\operatorname{ker} \rho$, where $\rho: \mathcal{S}_{1} \times p_{2}\left(K^{\prime}\right) \rightarrow \mathbb{Z}_{2}$ is the following representation:

$$
\rho(A, x)=\sigma_{1}(A) x \in \mathbb{Z}_{2} .
$$

Consider the lift of this representation $\widetilde{\rho}: \mathcal{S}_{2} \times p_{2}\left(K^{\prime}\right) \rightarrow \mathbb{Z}_{2}$. Similarly to the proof of Lemma 6.2 , we can easily show that this lift is only determined by $\sigma_{2}: \mathcal{S}_{2} \rightarrow \mathbb{Z}_{2}$ such that $\left.\sigma_{2}\right|_{\mathcal{S}_{1}}=\sigma_{1}$, i.e., a representation $\widetilde{\rho}: \mathcal{S}_{2} \times p_{2}\left(K^{\prime}\right) \rightarrow \mathbb{Z}_{2}$ is denoted by

$$
\widetilde{\rho}(B, x)=\sigma_{2}(B) x .
$$

On the other hand, there is the following induced representation form $K_{2}^{\prime} \subset \mathcal{S}_{2} \times$ $p_{2}\left(K^{\prime}\right)$ :

$$
\mathcal{S}_{2} \times p_{2}\left(K^{\prime}\right) \stackrel{\widetilde{r}}{\longrightarrow}\left(\mathcal{S}_{2} \times p_{2}\left(K^{\prime}\right)\right) / K_{2}^{\prime} \simeq \mathbb{Z}_{2} .
$$

With a method similar to that demonstrated in Section 6.1, $\widetilde{r}$ can be identified with $\widetilde{\rho}$, i.e., the lift of $\rho$. Hence, there is a representation $\sigma_{2}: \mathcal{S}_{2} \rightarrow \mathbb{Z}_{2}$ such that $\widetilde{r}(B, x)=\sigma_{2}(B) x$. Because ker $\widetilde{r}=K_{2}^{\prime}$, we have that

$$
=\left\{\left(B,\left(\begin{array}{cc}
X & 0 \\
0 & x
\end{array}\right)\right) \in \mathcal{S}_{2} \times S\left(O\left(2 k_{1}-1\right) \times O(1)\right) \mid \sigma_{2}(B) x=1\right\},
$$

where $\sigma_{2}: \mathcal{S}_{2} \rightarrow \mathbb{Z}_{2}$ is a representation such that $\left.\sigma_{2}\right|_{\mathcal{S}_{1}}=\sigma_{1}$.

If $\sigma_{1}\left(\mathcal{S}_{1}\right)=\{1\}$, then we have

$$
K=\prod_{i=1}^{a} S\left(U\left(l_{i}\right) \times U(1)\right) \times \mathcal{S}_{1} \times S O\left(2 k_{1}-1\right),
$$

by Lemma 7.1 (2). Because of the assumptions of the case (2)-(b)-(i), we have that

$$
K_{2}=\prod_{i=1}^{a} S\left(U\left(l_{i}\right) \times U(1)\right) \times \mathcal{S}_{2} \times S O\left(2 k_{1}-1\right) .
$$

Therefore, we can regard $K_{2}^{\prime}$ of this case as that with $\sigma_{2}\left(\mathcal{S}_{2}\right)=1$ in Eq.(7.1).

Hence, we have that $M=X_{1} \cup X_{2}$ is as follows, with the method similar to that demonstrated in Section 6.1:

$$
M=\prod_{i=1}^{a} \mathbb{C} P\left(l_{i}\right) \times\left(\prod_{j=1}^{b} S^{2 m_{j}} \times_{\mathcal{A} \times \mathbb{Z}_{2}} S\left(\mathbb{R}^{2 k_{1}} \oplus \mathbb{R}\right)\right),
$$


where the $\mathcal{A} \times \mathbb{Z}_{2}$-quotient is defined by the same rule as that in the case (2)(a)-(i) (see Section 6.1 by replacing $\mathbb{C}_{\mathfrak{c}}^{k_{1}}$ as $\mathbb{R}^{2 k_{1}}$ ), and $G=\prod_{i=1}^{a} S U\left(l_{i}+1\right) \times$ $\prod_{j=1}^{b} S O\left(2 m_{j}+1\right) \times S O\left(2 k_{1}\right)$ acts naturally on this manifold.

7.2. The case (2)-(b)-(ii). Suppose the case (2)-(b)-(ii) occurs, that is,

$$
p_{1}\left(K_{2}^{\prime}\right)=p_{1}\left(K^{\prime}\right)=\mathcal{S}_{1} \quad \text { and } \quad p_{2}\left(K_{2}^{\prime}\right) / p_{2}\left(K^{\prime}\right) \simeq \mathbb{Z}_{2} .
$$

If $\sigma_{1}\left(\mathcal{S}_{1}\right)=\{ \pm 1\}$, then we have $p_{2}\left(K^{\prime}\right)=S\left(O\left(2 k_{1}-1\right) \times O(1)\right) \supset p_{2}\left(K_{2}^{\prime}\right)$ by Lemma 7.1 (3). This gives a contradiction to $p_{2}\left(K_{2}^{\prime}\right) / p_{2}\left(K^{\prime}\right) \simeq \mathbb{Z}_{2}$. Therefore, we have $\sigma_{1}\left(\mathcal{S}_{1}\right)=\{1\}$. Hence, we have that $K^{\prime}=\mathcal{S}_{1} \times S O\left(2 k_{1}-1\right)$ by Lemma $7.1(2)$.

Because $p_{2}\left(K_{2}^{\prime}\right) / p_{2}\left(K^{\prime}\right) \simeq \mathbb{Z}_{2}$ and $p_{2}\left(K^{\prime}\right)=S O\left(2 k_{1}-1\right)$, we have the following sequence:

$$
K^{\prime}=\mathcal{S}_{1} \times S O\left(2 k_{1}-1\right) \subset K_{2}^{\prime} \subset \mathcal{S}_{1} \times p_{2}\left(K_{2}^{\prime}\right)=\mathcal{S}_{1} \times S\left(O\left(2 k_{1}-1\right) \times O(1)\right) .
$$

Because $K_{2}^{\prime} / K^{\prime} \simeq \mathbb{Z}_{2}$, we have that $K_{2}^{\prime}=\mathcal{S}_{1} \times S\left(O\left(2 k_{1}-1\right) \times O(1)\right)$ and

$$
\begin{aligned}
& K_{2}=\prod_{i=1}^{a} S\left(U\left(l_{i}\right) \times U(1)\right) \times \mathcal{S}_{1} \times S\left(O\left(2 k_{1}-1\right) \times O(1)\right), \\
& K=\prod_{i=1}^{a} S\left(U\left(l_{i}\right) \times U(1)\right) \times \mathcal{S}_{1} \times S O\left(2 k_{1}-1\right) .
\end{aligned}
$$

Hence, similarly to Section 7.1, we have that $M=X_{1} \cup X_{2}$ is as follows:

$$
M=\prod_{i=1}^{a} \mathbb{C} P\left(l_{i}\right) \times\left(\prod_{j=1}^{b} S^{2 m_{j}} \times_{\mathcal{A} \times \mathbb{Z}_{2}} S\left(\mathbb{R}^{2 k_{1}} \oplus \mathbb{R}\right)\right),
$$

where the $\mathcal{A} \times \mathbb{Z}_{2}$-quotient is defined by the same rule as that in the case (2)-(a)-(ii) (see Section 6.2 by replacing $\mathbb{C}_{\mathfrak{c}}^{k_{1}}$ as $\mathbb{R}^{2 k_{1}}$ ). This manifold is also $G$-equivariantly diffeomorphic to the following manifold:

$$
\prod_{i=1}^{a} \mathbb{C} P\left(l_{i}\right) \times \prod_{j=1}^{b} S^{2 m_{j}} / \mathcal{A} \times P\left(\mathbb{R}^{2 k_{1}} \oplus \mathbb{R}\right)
$$

where $G=\prod_{i=1}^{a} S U\left(l_{i}+1\right) \times \prod_{j=1}^{b} S O\left(2 m_{j}+1\right) \times S O\left(2 k_{1}\right)$.

7.3. The case (2)-(b)-(iii). Suppose the case (2)-(b)-(iii) occurs, that is,

$$
p_{1}\left(K_{2}^{\prime}\right)=p_{1}\left(K^{\prime}\right)=\mathcal{S}_{1} \quad \text { and } \quad p_{2}\left(K_{2}^{\prime}\right)=p_{2}\left(K^{\prime}\right) .
$$

If $\sigma_{1}\left(\mathcal{S}_{1}\right)=\{1\}$, then we have $K^{\prime}=\mathcal{S}_{1} \times S O\left(2 k_{1}-1\right)=p_{1}\left(K^{\prime}\right) \times p_{2}\left(K^{\prime}\right)=$ $p_{1}\left(K_{2}^{\prime}\right) \times p_{2}\left(K_{2}^{\prime}\right) \supset K_{2}^{\prime}$. This gives a contradiction to $K_{2}^{\prime} / K^{\prime} \simeq \mathbb{Z}_{2}$. Therefore, we have $\sigma_{1}\left(\mathcal{S}_{1}\right)=\{ \pm 1\}$.

Hence, we have the following sequence:

$$
K^{\prime} \subset K_{2}^{\prime} \subset p_{1}\left(K_{2}^{\prime}\right) \times p_{2}\left(K_{2}^{\prime}\right)=\mathcal{S}_{1} \times S\left(O\left(2 k_{1}-1\right) \times O(1)\right) .
$$

By Lemma $7.1(3)$ and $K_{2}^{\prime} / K^{\prime} \simeq \mathbb{Z}_{2}$, we also have that $K_{2}^{\prime}=\mathcal{S}_{1} \times S\left(O\left(2 k_{1}-1\right) \times\right.$ $O(1))$ and

$$
\begin{aligned}
& K_{2}=\prod_{i=1}^{a} S\left(U\left(l_{i}\right) \times U(1)\right) \times \mathcal{S}_{1} \times S\left(O\left(2 k_{1}-1\right) \times O(1)\right), \\
& K=\prod_{i=1}^{a} S\left(U\left(l_{i}\right) \times U(1)\right) \times\left\{(A, x) \mid \sigma_{1}(A) x=1\right\} .
\end{aligned}
$$


Hence, similarly to Section 7.1, we have that $M=X_{1} \cup X_{2}$ is as follows:

$$
M=\prod_{i=1}^{a} \mathbb{C} P\left(l_{i}\right) \times\left(\prod_{j=1}^{b} S^{2 m_{j}} \times_{\mathcal{A} \times \mathbb{Z}_{2}} S\left(\mathbb{R}^{2 k_{1}} \oplus \mathbb{R}\right)\right),
$$

where the $\mathcal{A} \times \mathbb{Z}_{2}$-quotient is defined by the same rule as that in the case (2)-(a)-(iii) (see Section 6.3 by replacing $\mathbb{C}_{\mathfrak{c}}^{k_{1}}$ as $\mathbb{R}^{2 k_{1}}$ ). This manifold is also $G$-equivariantly diffeomorphic to the following manifold as well as the case (2)-(b)-(ii):

$$
\prod_{i=1}^{a} \mathbb{C} P\left(l_{i}\right) \times \prod_{j=1}^{b} S^{2 m_{j}} \times_{\mathcal{A}} P\left(\mathbb{R}^{2 k_{1}} \oplus \mathbb{R}\right) .
$$

7.4. The case (2)-(b)-(iv). Suppose the case (2)-(b)-(iv) occurs, that is,

$$
p_{1}\left(K_{2}^{\prime}\right)=\mathcal{S}_{2} \text { and } p_{2}\left(K_{2}^{\prime}\right) / p_{2}\left(K^{\prime}\right) \simeq \mathbb{Z}_{2} .
$$

By the same reason in the case (2)-(b)-(ii) (see Section 7.2), we have $\sigma_{1}\left(\mathcal{S}_{1}\right)=$ $\{1\}$. Hence, we have that $K^{\prime}=\mathcal{S}_{1} \times S O\left(2 k_{1}-1\right)$ by Lemma $7.1(2)$.

Because $p_{2}\left(K_{2}^{\prime}\right) / p_{2}\left(K^{\prime}\right) \simeq \mathbb{Z}_{2}$ and $p_{2}\left(K^{\prime}\right)=S O\left(2 k_{1}-1\right)$, we have the following sequence:

$$
K^{\prime}=\mathcal{S}_{1} \times S O\left(2 k_{1}-1\right) \subset K_{2}^{\prime} \subset \mathcal{S}_{2} \times p_{2}\left(K_{2}^{\prime}\right)=\mathcal{S}_{2} \times S\left(O\left(2 k_{1}-1\right) \times O(1)\right) .
$$

Because $K_{2}^{\prime} / K^{\prime} \simeq \mathbb{Z}_{2}$, we also have the following inclusion map:

$$
i: K_{2}^{\prime} / K^{\prime} \simeq \mathbb{Z}_{2} \longrightarrow\left(\mathcal{S}_{2} \times S\left(O\left(2 k_{1}-1\right) \times O(1)\right)\right) / K^{\prime} \simeq \mathbb{Z}_{2} \times \mathbb{Z}_{2} .
$$

Therefore, there are three types of the inclusion $i$, i.e., $i\left(K_{2}^{\prime} / K^{\prime}\right)=\mathbb{Z}_{2} \times\{1\},\{1\} \times \mathbb{Z}_{2}$ or $\Delta$, where $\Delta$ is the diagonal subgroup in $\mathbb{Z}_{2} \times \mathbb{Z}_{2}$. Assume $i\left(K_{2}^{\prime} / K^{\prime}\right)=\mathbb{Z}_{2} \times\{1\}$ or $\{1\} \times \mathbb{Z}_{2}$. This gives a contradiction to $p_{2}\left(K_{2}^{\prime}\right) / p_{2}\left(K^{\prime}\right) \simeq \mathbb{Z}_{2}$ or $p_{1}\left(K_{2}^{\prime}\right) / p_{1}\left(K^{\prime}\right) \simeq \mathbb{Z}_{2}$, respectively. Therefore, we have that

$$
i\left(K_{2}^{\prime} / K^{\prime}\right)=\Delta .
$$

Let $\widetilde{\rho}: \mathcal{S}_{2} \times p_{2}\left(K_{2}^{\prime}\right) \rightarrow \mathbb{Z}_{2} \times \mathbb{Z}_{2}$ be a representation such that $\widetilde{\rho}(B, x)=\left(\sigma_{2}(B), x\right)$ for some $\sigma_{2}: \mathcal{S}_{2} \rightarrow \mathbb{Z}_{2}$ with $\left.\sigma_{2}\right|_{\mathcal{S}_{1}}=\sigma_{1}$. By $(7.2)$, we have that $K_{2}^{\prime}=\widetilde{\rho}^{-1}(\Delta)$. It follows that

$$
K_{2}^{\prime}=\left\{\left(B,\left(\begin{array}{cc}
X & 0 \\
0 & x
\end{array}\right)\right) \in \mathcal{S}_{2} \times S\left(O\left(2 k_{1}-1\right) \times O(1)\right) \mid \sigma_{2}(B) x=1\right\} .
$$

Because $K_{2}=\prod_{i=1}^{a} S\left(U\left(l_{i}\right) \times U(1)\right) \times K_{2}^{\prime}$ and $K=\prod_{i=1}^{a} S\left(U\left(l_{i}\right) \times U(1)\right) \times$ $\mathcal{S}_{1} \times S O\left(2 k_{1}-1\right)$, (similarly to Section 7.1) we have that $M=X_{1} \cup X_{2}$ is as follows:

$$
M=\prod_{i=1}^{a} \mathbb{C} P\left(l_{i}\right) \times\left(\prod_{j=1}^{b} S^{2 m_{j}} \times_{\mathcal{A} \times \mathbb{Z}_{2}} S\left(\mathbb{R}^{2 k_{1}} \oplus \mathbb{R}\right)\right),
$$

where the $\mathcal{A} \times \mathbb{Z}_{2}$-quotient is defined by the same rule as that in the case (2)-(a)-(iv) (see Section 6.4 by replacing $\mathbb{C}_{\mathfrak{c}}^{k_{1}}$ as $\mathbb{R}^{2 k_{1}}$ ). 
7.5. Summary of the results from (2)-(b)-(i) to (iv). In summary, we can state the result of the case (2)-(b) as follows. Let $(M, G)$ be the pair in the case (2)-(b). Then $(M, G)$ is essentially isomorphism to the followings:

$$
\begin{aligned}
& M=\prod_{j=1}^{b} S^{2 m_{j}} \times_{\mathcal{A} \times \mathbb{Z}_{2}}\left(\prod_{i=1}^{a} \mathbb{C} P\left(l_{i}\right) \times S\left(\mathbb{R}^{2 k_{1}} \oplus \mathbb{R}\right)\right), \\
& G=\prod_{j=1}^{b} S O\left(2 m_{j}+1\right) \times \prod_{i=1}^{a} S U\left(l_{i}+1\right) \times S O\left(2 k_{1}\right),
\end{aligned}
$$

where $G$ acts on $M$ naturally, and the $\mathcal{A}\left(=\mathcal{A}_{1}\right)$-quotient is defined by the following actions:

- on $\prod_{j=1}^{b} S^{2 m_{j}}$ as the subgroup of $\prod_{j=1}^{b} \mathbb{Z}_{2}$;

- on $\mathbb{R}^{2 k_{1}}$ through the representation $\sigma_{1}\left(=\sigma_{\mathbb{R}}\right): \mathcal{A} \rightarrow \mathbb{Z}_{2}=\left\{ \pm I_{2 k_{1}}\right\}$;

- on $\mathbb{R}$ trivially,

and the $\mathbb{Z}_{2}$-quotient is defined by the following actions:

- on $\prod_{j=1}^{b} S^{2 m_{j}}$ by a representation $\rho: \mathbb{Z}_{2} \rightarrow \prod_{j=1}^{b} \mathbb{Z}_{2}$ which satisfies $\rho\left(\mathbb{Z}_{2}\right) \cap \mathcal{A}=\{1\}$

- on $\mathbb{R}^{2 k_{1}}$ by a representation $\sigma_{2}: \mathbb{Z}_{2} \rightarrow\left\{ \pm I_{2 k_{1}}\right\}$;

- on $\mathbb{R}$ by the natural representation $\kappa: \mathbb{Z}_{2} \rightarrow O(1)$,

where $\rho$ or $\sigma_{2}$ is always non-trivial as well as the case (2)-(a). This corresponds with the second manifold in Theorem 5.1, where $\sigma_{2} \oplus \kappa=\sigma_{\mathbb{R} \oplus \mathbb{R}}$.

\section{Main theorem of the case (3) and preparations}

From this section, we start to classify the final case, i.e., the case (3): $G / K_{2}$ is not a torus manifold but a singular orbit (see Section 2.4). The goal of this section is to state the main theorem and a preparation to classify the case (3). From this section, we assume that the orbit $G / K_{2}$ is not a torus manifold but a singular orbit. By Lemma 4.1 and the assumption of this case, we have for $k_{2} \geq 2$

$$
\operatorname{dim} G / K_{2}=2 n-2 k_{2}+1,
$$

and

$$
K_{2} / K \cong S^{2 k_{2}-2} .
$$

By using (8.1) and (8.2), the slice representation of $K_{2}$ in the case (3) is

$$
\sigma_{2}: K_{2} \longrightarrow O\left(2 k_{2}-1\right) \text {. }
$$

In the case (3) as well as the case (2), there are the following two cases:

(3)-(a): the case (3)-(a), i.e., $G / K_{1}$ satisfies the case (a) (see Section 4.1.1); (3)-(b): the case (3)-(b), i.e., $G / K_{1}$ satisfies the case (b) (see Section 4.1.2).

8.1. Main theorem and notations. First we state the main theorem of the case (3). Before we state it, we prepare some notations (also see Section 3.1 and 5.1). Let $\mathcal{A}$ be a subgroup of $\prod_{j=1}^{b} \mathbb{Z}_{2}$, where $\prod_{j=1}^{b} \mathbb{Z}_{2}$ is the following group: the first $(b-1)$ factors $\prod_{j=1}^{b-1} \mathbb{Z}_{2}$ are generated by the antipodal involutions on $S^{2 m_{j}}$ for $j=1, \cdots, b-1$ and the $b$-th factor $\mathbb{Z}_{2}$ is $\left\{ \pm I_{2 k_{2}-1}\right\}$. The quotient manifold $\left(\prod_{i=1}^{a} S^{2 l_{i}+1}\right) \times_{T^{a}} S\left(\mathbb{C}_{\mathfrak{c}}^{k_{1}} \oplus \mathbb{R}^{2 k_{2}-1}\right)$ is defined similarly as $\left(\prod_{i=1}^{a} S^{2 l_{i}+1}\right) \times_{T^{a}} S\left(\mathbb{C}_{\mathfrak{a}}^{k} \oplus\right.$ 
$\mathbb{R})($ see Section 3.1$)$, where $\mathbb{C}_{\mathfrak{c}}^{k_{1}}\left(\simeq \mathbb{C}^{k_{1}}\right)$ is a representation space of a representation c : $T^{a} \rightarrow S^{1}$.

Now we may state the main theorem of this section.

THEOREM 8.1. Suppose a torus manifold $M$ has a codimension one extended $G$-action. If there are two singular orbits and one of them is not a torus manifold, then $(M, G)$ is essentially isomorphic to

$$
\left(\prod_{j=1}^{b-1} S^{2 m_{j}} \times_{\mathcal{A}} N, \quad \prod_{j=1}^{b-1} S O\left(2 m_{j}+1\right) \times H\right)
$$

such that $(N, H)$ is one of the followings:

\begin{tabular}{|c|c|c|}
\hline & $N$ & $H$ \\
\hline \hline$(a)$ & $\left(\prod_{i=1}^{a} S^{2 l_{i}+1}\right) \times \times_{T^{a}} S\left(\mathbb{C}_{\mathfrak{c}}^{k_{1}} \oplus \mathbb{R}^{2 k_{2}-1}\right)$ & $\prod_{i=1}^{a} S U\left(l_{i}+1\right) \times U\left(k_{1}\right) \times S O\left(2 k_{2}-1\right)$ \\
\hline (b) & $\prod_{i=1}^{a} \mathbb{C} P\left(l_{i}\right) \times S\left(\mathbb{R}^{2 k_{1}} \oplus \mathbb{R}^{2 k_{2}-1}\right)$ & $\prod_{i=1}^{a} S U\left(l_{i}+1\right) \times S O\left(2 k_{1}\right) \times S O\left(2 k_{2}-1\right)$ \\
\hline
\end{tabular}

where $\mathcal{A}$ acts on $\prod_{j=1}^{b-1} S^{2 m_{j}} \times \mathbb{R}^{2 k_{2}-1}$ as a subgroup of $\prod_{j=1}^{b} \mathbb{Z}_{2}$ and on the fibre of $N$ through the following representations:

(a): $\sigma_{\mathbb{C}}: \mathcal{A} \rightarrow\{ \pm 1\} \subset S^{1}$ on $S\left(\mathbb{C}_{\mathfrak{c}}^{k_{1}} \oplus \mathbb{R}^{2 k_{2}-1}\right) \cap \mathbb{C}_{\mathfrak{c}}^{k_{1}} ;$

(b): $\sigma_{\mathbb{R}}: \mathcal{A} \rightarrow\left\{ \pm I_{2 k_{1}}\right\} \subset S O\left(2 k_{1}\right)$ on $S\left(\mathbb{R}^{2 k_{1}} \oplus \mathbb{R}^{2 k_{2}-1}\right) \cap \mathbb{R}^{2 k_{1}}$;

respectively, such that if $\left(1, \cdots, 1,-I_{2 k_{2}-1}\right) \in \mathcal{A} \subset \prod_{j=1}^{b} \mathbb{Z}_{2}$ then

$$
\sigma\left(1, \cdots, 1,-I_{2 k_{2}-1}\right)=-1
$$

for $\sigma=\sigma_{\mathbb{C}}$ and $\sigma_{\mathbb{R}}$.

Here, $G$-actions on $M$ are as follows: $\prod S O\left(2 m_{j}+1\right)$ and $\prod S U\left(l_{i}+1\right)$ act naturally on $\prod S^{2 m_{j}}$ and $\prod S^{2 l_{i}+1}$, respectively; and $U\left(k_{1}\right), S O\left(2 k_{1}\right)$ and $S O\left(2 k_{2}-\right.$ 1) act naturally on $\mathbb{C}_{\mathfrak{c}}^{k_{1}}, \mathbb{R}^{2 k_{1}}$ and $\mathbb{R}^{2 k_{2}-1}$, respectively.

Note that the following facts: if $\left(1, \cdots, 1,-I_{2 k_{2}-1}\right) \notin \mathcal{A}$ then $\mathcal{A}$ acts on $\prod_{j=1}^{b-1} S^{2 m_{j}} \times \mathbb{R}^{2 k_{2}-1}$ freely; if $\left(1, \cdots, 1,-I_{2 k_{2}-1}\right) \in \mathcal{A}$ then $\mathcal{A}=\mathcal{A}^{\prime} \times\left\{ \pm I_{2 k_{2}-1}\right\}$ and $\mathcal{A}^{\prime}$ acts on $\prod_{j=1}^{b-1} S^{2 m_{j}}$ freely and $\left\{ \pm I_{2 k_{2}-1}\right\}$ acts on $S\left(\mathbb{C}_{\mathfrak{c}}^{k_{1}} \oplus \mathbb{R}^{2 k_{2}-1}\right)$ or $S\left(\mathbb{R}^{2 k_{1}} \oplus\right.$ $\mathbb{R}^{2 k_{2}-1}$ ) freely because of the properties of $\sigma$ described in Theorem 8.1. Therefore, $M$ in Theorem 8.1 is a manifold. Moreover, there is the case that $\mathcal{A} \subset \prod_{j=1}^{b-1} \mathbb{Z}_{2}$; hence, we do not write manifolds in Theorem 8.1 as manifolds in Theorem 5.1, i.e., manifolds divided by $\mathcal{A} \times \mathbb{Z}_{2}$ where $\mathbb{Z}_{2}$ acts on $S\left(\mathbb{C}_{\mathfrak{c}}^{k_{1}} \oplus \mathbb{R}\right) \cap \mathbb{R}\left(\right.$ or $\left.S\left(\mathbb{R}^{2 k_{1}} \oplus \mathbb{R}\right) \cap \mathbb{R}\right)$.

In order to prove the above Theorem 8.1, we will use the following notations.

- Natural projections: $p_{i}: G \rightarrow S U\left(l_{i}+1\right), p: G \rightarrow S U\left(k_{1}\right), q: G \rightarrow T^{1}$ and $\quad r_{j}: G \rightarrow S O\left(2 m_{j}+1\right)$, where $i=1, \cdots, a$ and $j=1, \cdots, b$.

- Inclusions: $\iota: K \rightarrow K_{2}$ or $\iota: K^{o} \rightarrow K_{2}^{o}$, and $\iota_{2}: K_{2} \rightarrow G$ or $\iota_{2}: K_{2}^{o} \rightarrow G$.

8.2. Structure of $K_{2}$. Before we start to prove Theorem 8.1, in this subsection, we will prove the following Lemma 8.2.

Let $N_{1} \circ N_{2}$ be $\left(N_{1} \times N_{2}\right) / F$ for some finite, normal subgroup $F \subset N_{1} \times N_{2}$, where $N_{1}$ and $N_{2}$ are connected Lie groups. Then, the following lemma holds.

Lemma 8.2. For the cases (3)-(a) and (3)-(b), the pair $\left(K_{2}^{o}, K^{o}\right)$ is isomorphic to

$$
\left(\operatorname{Spin}\left(2 k_{2}-1\right) \circ K_{2}^{\prime}, \operatorname{Spin}\left(2 k_{2}-2\right) \circ K_{2}^{\prime}\right)
$$

for some Lie group $K_{2}^{\prime}$ and $k_{2} \geq 2$. 
Proof. Let $\widetilde{K_{2}^{o}}$ be the covering of $K_{2}^{o}$ such that it is a product of simply connected, simple Lie groups and tori (see [12, Section 2.3]). Since a connected component $K_{2}^{o}$ acts on $S^{2 k_{2}-2} \cong K_{2} / K$ transitively through $\sigma_{2}$ (see (8.3)), there is a factor $H$ in the product group $\widetilde{K_{2}^{o}}$ (i.e., $\widetilde{K_{2}^{o}}=H \times \widetilde{K_{2}^{\prime}}$ for some product group $\widetilde{K_{2}^{\prime}}$ ) such that $H=\operatorname{Spin}\left(2 k_{2}-1\right)$ or $H=G_{2}$ for $k_{2}=4$ by the classification result of transitive actions on even dimensional spheres (see [13, Theorem 5.1, 5.2]), where here $G_{2}$ is the exceptional Lie group. Therefore, there is a subgroup $K_{2}^{\prime}$ such that $\left(K_{2}^{o}, K^{o}\right)$ can be denoted as follows:

$$
\begin{aligned}
& \left(\operatorname{Spin}\left(2 k_{2}-1\right) \circ K_{2}^{\prime}, \operatorname{Spin}\left(2 k_{2}-2\right) \circ K_{2}^{\prime}\right) ; \\
& \left(G_{2} \circ K_{2}^{\prime}, S U(3) \circ K_{2}^{\prime}\right) .
\end{aligned}
$$

In order to prove this lemma, we assume

$$
\left(K_{2}^{o}, K^{o}\right)=\left(G_{2} \circ K_{2}^{\prime}, S U(3) \circ K_{2}^{\prime}\right) .
$$

We will prove that this case does not occur.

Taking some covering of $K^{o}$ in (8.4), we can put

$$
\widetilde{K^{o}}=S U(3) \times \widetilde{K_{2}^{\prime}},
$$

where $\widetilde{K_{2}^{\prime}}$ is a product of simply connected, simple Lie groups and tori. On the other hand, taking a covering of $K^{o}$ in the cases (3)-(a) and (3)-(b) (see Section 4.1.1, 4.1.2 or (9.1) in Section 9, (10.1) in Section 10), we can put

$$
\widetilde{K^{o}}=\prod_{j=1}^{b} \operatorname{Spin}\left(2 m_{j}\right) \times \prod_{i=1}^{a} S U\left(l_{i}\right) \times L \times T,
$$

where $T$ is a torus and

- $L=S U\left(k_{1}-1\right)$ for the case (3)-(a),

- $L=\operatorname{Spin}\left(2 k_{1}-1\right)$ for the case $(3)-(\mathrm{b})$.

Because $\operatorname{dim} \operatorname{Spin}(x) \neq \operatorname{dim} S U(3)$ for all $x \in \mathbb{N}$, there are the following two cases by (8.5) and (8.6):

(1) $l_{a}=3$ and $\widetilde{K_{2}^{\prime}}=\prod_{j=1}^{b} \operatorname{Spin}\left(2 m_{j}\right) \times \prod_{i=1}^{a-1} S U\left(l_{i}\right) \times L \times T$, in the case (3)-(a) or (3)-(b);

(2) $k_{1}=4$ and $\widetilde{K_{2}^{\prime}}=\prod_{j=1}^{b} \operatorname{Spin}\left(2 m_{j}\right) \times \prod_{i=1}^{a} S U\left(l_{i}\right) \times T$, in the case (3)-(a).

Suppose $l_{a}=3$ and $\widetilde{K_{2}^{\prime}}=\prod_{j=1}^{b} \operatorname{Spin}\left(2 m_{j}\right) \times \prod_{i=1}^{a-1} S U\left(l_{i}\right) \times L \times T$. Let $p_{a}: G \rightarrow S U\left(l_{a}+1\right)$ be the natural projection (see notations in Section 8.1). Then we have that

$$
p_{a}(S U(3) \circ\{e\}) \subset p_{a}\left(G_{2} \circ\{e\}\right) \subset p_{a}\left(K_{2}^{o}\right) \subset p_{a}(G)=S U(4)
$$

because $S U(3) \circ\{e\} \subset G_{2} \circ\{e\} \subset K_{2}^{o} \subset G$, where $\{e\} \subset K_{2}^{\prime}$ is the identity element in $K_{2}^{\prime}$. Since $S U(3) \circ\{e\} \subset K^{o}$ and $p_{a}\left(K^{o}\right)=S(U(3) \times U(1))$ by Section 4.1, we also have that

$$
p_{a}(S U(3) \circ\{e\})=S U(3) .
$$

Therefore, $p_{a}\left(G_{2} \circ\{e\}\right)$ is a non-trivial subgroup in $S U(4)$. Since the restricted representation $\left.p_{a}\right|_{G_{2} \circ\{e\}}$ is a non-trivial representation and $G_{2}$ is a simple Lie group, we also have that

$$
\operatorname{dim} p_{a}\left(G_{2} \circ\{e\}\right)=\operatorname{dim} G_{2}=14 .
$$


It follows that there is a subgroup $H \subset S U(4)$ such that $\operatorname{dim} H=14$ and $S U(4) / H \cong$ $S^{1}$, because $S U(4)$ is compact and $\operatorname{dim} S U(4)=15$. However, this gives a contradiction because $S U(4)$-action on $S^{1}$ is trivial (see [13, Theorem 5.2, 5.3]).

Suppose $k_{1}=4$ and $\widetilde{K_{2}^{\prime}}=\prod_{j=1}^{b} \operatorname{Spin}\left(2 m_{j}\right) \times \prod_{i=1}^{a} S U\left(l_{i}\right) \times T$. In this case, the above argument for $l_{a}=3$ can also work for the natural projection $p: G \rightarrow S U\left(k_{1}\right)$. It follows that the case $\left(K_{2}^{o}, K^{o}\right)=\left(G_{2} \circ K_{2}^{\prime}, S U(3) \circ K_{2}^{\prime}\right)$ does not occur. Hence, we have Lemma 8.2.

In the next two sections, we study the cases (3)-(a) and (3)-(b).

\section{The case (3)-(a)}

In this section, we study the case (3)-(a). From Section 4.1, we have

$$
\begin{aligned}
& G=\prod_{j=1}^{b} S O\left(2 m_{j}+1\right) \times \prod_{i=1}^{a} S U\left(l_{i}+1\right) \times S U\left(k_{1}\right) \times T^{1}, \\
& K_{1}=\mathcal{S}_{1} \times \prod_{i=1}^{a} S\left(U\left(l_{i}\right) \times U(1)\right) \times S U\left(k_{1}\right) \times T^{1}, \\
& K=\left\{\left(A,\left(t_{1}, \cdots, t_{a}\right),\left(\begin{array}{cc}
X & 0 \\
0 & x
\end{array}\right), t\right) \mid \sigma_{1}(A) t_{1}^{\alpha_{1}} \cdots t_{a}^{\alpha_{a}} x t^{\alpha}=1\right\} .
\end{aligned}
$$

where $A \in \mathcal{S}_{1} \subset \prod_{j=1}^{b} S\left(O\left(2 m_{j}\right) \times O(1)\right),\left(t_{1}, \cdots, t_{a}\right) \in \prod_{i=1}^{a} S\left(U\left(l_{i}\right) \times U(1)\right)$, $t \in T^{1}$ and $X \in U\left(k_{1}-1\right)$ such that $x \operatorname{det} X=1$. If $k_{1}=1$, then we can take $\alpha=1$. Moreover, we have that the finite covering of $K^{o}$ is as follows by (9.1):

$$
\begin{aligned}
\widetilde{K^{o}} & =\prod_{j=1}^{b} \operatorname{Spin}\left(2 m_{j}\right) \times \prod_{i=1}^{a} S U\left(l_{i}\right) \times T^{a} \times S U\left(k_{1}-1\right) \times T^{1} \quad \text { if } k_{1} \geq 2 ; \\
\widetilde{K^{o}} & =\prod_{j=1}^{b} \operatorname{Spin}\left(2 m_{j}\right) \times \prod_{i=1}^{a} S U\left(l_{i}\right) \times T^{a} \quad \text { if } k_{1}=1 .
\end{aligned}
$$

Because of Lemma 8.2, we also have

$$
\begin{aligned}
& K^{o}=\operatorname{Spin}\left(2 k_{2}-2\right) \circ K_{2}^{\prime} \quad \text { and } \quad \widetilde{K^{o}}=\operatorname{Spin}\left(2 k_{2}-2\right) \times \widetilde{K_{2}^{\prime}}, \\
& K_{2}^{o}=\operatorname{Spin}\left(2 k_{2}-1\right) \circ K_{2}^{\prime} \quad \text { and } \quad \widetilde{K_{2}^{o}}=\operatorname{Spin}\left(2 k_{2}-1\right) \times \widetilde{K_{2}^{\prime}} .
\end{aligned}
$$

In order to classify the case (3)-(a), we will divide this case into the following two cases:

- $k_{2} \geq 3$ (we will discuss in Section 9.1);

- $k_{2}=2$ (we will discuss in Section 9.2).

9.1. The case $k_{2} \geq 3$. Assume $k_{2} \geq 3$. Comparing coverings $\widetilde{K^{\circ}}$ of the above $K^{o}$ 's in (9.1) and (9.2), and using the fact that $\operatorname{Spin}(4) \simeq S U(2) \times S U(2)$ and $\operatorname{Spin}(6) \simeq S U(4)$, there are the following five cases:

(i): $\operatorname{Spin}\left(2 k_{2}-2\right)=\operatorname{Spin}\left(2 m_{b}\right)$, and $k_{2}=m_{b}+1 \geq 3$;

(ii): $\operatorname{Spin}\left(2 k_{2}-2\right)=S U\left(l_{a-1}\right) \times S U\left(l_{a}\right)$, and $k_{2}=3, l_{a-1}=l_{a}=2$;

(iii): $\operatorname{Spin}\left(2 k_{2}-2\right)=S U\left(l_{a}\right) \times S U\left(k_{1}-1\right)$, and $k_{2}=3=k_{1}, l_{a}=2$;

(iv): $\operatorname{Spin}\left(2 k_{2}-2\right)=S U\left(l_{a}\right)$ and $k_{2}=4, l_{a}=4$;

(v): $\operatorname{Spin}\left(2 k_{2}-2\right)=S U\left(k_{1}-1\right)$ and $k_{2}=4, k_{1}=5$, 
First we prove the following lemma.

LEMmA 9.1. In the above cases, the cases from (ii) to (v) do not occur.

Proof. If the case (ii) occurs, then $\operatorname{Spin}\left(2 k_{2}-2\right)=S U\left(l_{a-1}\right) \times S U\left(l_{a}\right)$ and $k_{2}=3, l_{a-1}=l_{a}=2$. Let $\varphi: \widetilde{K_{2}^{o}} \rightarrow K_{2}^{o}$ be the finite covering. By using (9.1), (9.2) and (9.3), we have

$$
\begin{aligned}
K_{2}^{\prime}= & \prod_{j=1}^{b} S O\left(2 m_{j}\right) \times\left\{\left(\left(\begin{array}{cc}
A_{1} & 0 \\
0 & t_{1}
\end{array}\right), \cdots,\left(\begin{array}{cc}
A_{a-2} & 0 \\
0 & t_{a-2}
\end{array}\right),\right.\right. \\
& \left.\left.\left(\begin{array}{cc}
r_{a-1} I_{2} & 0 \\
0 & t_{a-1}
\end{array}\right),\left(\begin{array}{cc}
r_{a} I_{2} & 0 \\
0 & t_{a}
\end{array}\right),\left(\begin{array}{cc}
X & 0 \\
0 & x
\end{array}\right), t\right) \mid t_{1}^{\alpha_{1}} \cdots t_{a}^{\alpha_{a}} x t^{\alpha}=1\right\} \\
= & \varphi\left(\widetilde{K_{2}^{\prime \prime}} \times T^{2}\right),
\end{aligned}
$$

where $r_{a-1}^{-2}=t_{a-1}, r_{a}^{-2}=t_{a}, \widetilde{K_{2}^{\prime \prime}}$ is the product of factors in $\widetilde{K^{o}}$ except $\operatorname{Spin}\left(2 k_{2}-\right.$ 2) and $T^{2}\left(\subset T^{a}\right)$. By $(9.2),(9.3),\left[\mathbf{1 2}\right.$, Section 3.1] (i.e., for the factor $H \subset \widetilde{K^{o}}$ in $H^{\prime} \subset \widetilde{K_{2}^{o}}$ such that $H \subset H^{\prime}$, if $\widetilde{K^{o}}$ and $\widetilde{K_{2}^{o}}$ are same rank then $H$ and $H^{\prime}$ are same rank) and the assumption of the case (ii), we have the following commutative diagram:

$$
\begin{array}{ccc}
\left(T^{2} \times S U(2) \times S U(2)\right) \times \widetilde{K_{2}^{\prime \prime}} & \stackrel{\tau}{\longrightarrow} & \left(T^{2} \times S p i n(5)\right) \times \widetilde{K_{2}^{\prime \prime}} \\
\varphi \downarrow & & \downarrow \varphi \\
K^{o} & \stackrel{\iota}{\longrightarrow} & K_{2}^{o},
\end{array}
$$

where $\widetilde{\iota}$ is an inclusion map. By using the above diagram and the definition of $K$ and $G$, we have the following sequence (also see Section 8.1 about the definitions of notations):

$$
\begin{aligned}
& S(U(2) \times U(1)) \times S(U(2) \times U(1)) \\
= & \left(p_{a-1} \times p_{a}\right) \circ \iota_{2} \circ \iota \circ \varphi\left(T^{2} \times S U(2) \times S U(2)\right) \\
= & \left(p_{a-1} \times p_{a}\right) \circ \iota_{2} \circ \varphi \circ \widetilde{\imath}\left(T^{2} \times S U(2) \times S U(2)\right) \\
\subset \quad & \left(p_{a-1} \times p_{a}\right) \circ \iota_{2} \circ \varphi\left(T^{2} \times S p i n(5)\right) \\
\subset & p_{a-1} \times p_{a}(G) \\
= & S U(3) \times S U(3) .
\end{aligned}
$$

This sequence implies that there is a non-trivial representation from $T^{2} \times \operatorname{Spin}(5)$ to $S U(3) \times S U(3)$. Since $\operatorname{Spin}(5)$ is a simple Lie group and $\operatorname{rank}\left(T^{2} \times \operatorname{Spin}(5)\right)=$ rank $(S U(3) \times S U(3))$, there is some subgroup $H \subset S U(3)$ such that $S p i n(5) \approx H$ (because of [12, Section 3.1]), where $\operatorname{Spin}(5) \approx H$ means that $\operatorname{Spin}(5)$ and $H$ have the same Lie algebra. This gives a contradiction, because $\operatorname{dim} \operatorname{Spin}(5)=\operatorname{dim} H=$ $10>8=\operatorname{dim} S U(3)$. Hence, the case (ii) does not occur.

With an argument similar to the above for the case (ii), we can also prove that the cases (iii) does not occur.

If the case (iv) occurs, then $\operatorname{Spin}\left(2 k_{2}-2\right)=S U\left(l_{a}\right)$ and $k_{2}=4, l_{a}=4$. With a method similar to that demonstrated in the proof of the case (ii), we have the following commutative diagram:

$$
\begin{array}{ccc}
\left(T^{1} \times S U(4)\right) \times \widetilde{K_{2}^{\prime \prime}} & \stackrel{\tau}{\longrightarrow} & \left(T^{1} \times \operatorname{Spin}(7)\right) \times \widetilde{K_{2}^{\prime \prime}} \\
\varphi \downarrow & & \downarrow \varphi \\
K^{o} & \stackrel{\iota}{\longrightarrow} & K_{2}^{o} .
\end{array}
$$


We have the following sequence by using the above diagram and the definitions of $K$ and $G$ :

$$
\begin{aligned}
& S(U(4) \times U(1)) \\
= & p_{a} \circ \iota_{2} \circ \iota \circ \varphi\left(T^{1} \times S U(4)\right) \\
= & p_{a} \circ \iota_{2} \circ \varphi \circ \widetilde{\iota}\left(T^{1} \times S U(4)\right) \\
\subset & p_{a} \circ \iota_{2} \circ \varphi\left(T^{1} \times \operatorname{Spin}(7)\right) \\
= & H \\
\subset & p_{a}(G)=S U(5) .
\end{aligned}
$$

Because $S(U(4) \times U(1)), S U(5)$ and $T^{1} \times \operatorname{Spin}(7)$ are the same rank Lie groups and $\operatorname{Spin}(7)$ is a simple Lie group, we have $\operatorname{dim} H=\operatorname{dim}\left(T^{1} \times \operatorname{Spin}(7)\right)=22$. Then we see $S U(5) / H$ is a 2 -dimensional manifold by $\operatorname{dim} S U(5)=24$. Moreover, $H=p_{a} \circ \iota_{2} \circ \varphi\left(T^{1} \times \operatorname{Spin}(7)\right)$ is connected. Therefore, we have $S U(5) / H$ is a simply connected, compact manifold, because of the homotopy exact sequence of $H \rightarrow S U(5) \rightarrow S U(5) / H$. Hence, $S U(5) / H \cong S^{2}$. However, the $S U(5)$-action on $S^{2}$ must be trivial (see [13, Theorem 5.2]). This gives a contradiction. Hence, the case (iv) does not occur.

With a method similar to that demonstrated in the above for the case (iv), we can also prove that the case (v) does not occur.

Because of the above Lemma 9.1, we have that

$$
\operatorname{Spin}\left(2 k_{2}-2\right)=\operatorname{Spin}\left(2 m_{b}\right) \quad \text { and } \quad k_{2}=m_{b}+1 \geq 3 .
$$

Now we set

$\left\{\left(\left(t_{1}, \cdots, t_{a}\right),\left(\begin{array}{cc}X & 0 \\ 0 & x\end{array}\right), t\right) \mid t_{1}^{\alpha_{1}} \cdots t_{a}^{\alpha_{a}} x t^{\alpha}=1\right\}=P\left(\alpha_{1}, \cdots, \alpha_{a}, x, \alpha\right)$.

If $k_{1}=1$, then $x=1$ and we can assume $\alpha=1$ up to essential isomorphism. Therefore, $P\left(\alpha_{1}, \cdots, \alpha_{a}, x, \alpha\right)$ is connected. Then, the following relation holds by using (9.1), (9.2) and (9.4):

$$
\begin{aligned}
K^{o} & =\operatorname{Spin}\left(2 k_{2}-2\right) \circ K_{2}^{\prime}=\operatorname{Spin}\left(2 m_{b}\right) \circ K_{2}^{\prime} \\
& =\prod_{j=1}^{b} S O\left(2 m_{j}\right) \times P\left(\alpha_{1}, \cdots, \alpha_{a}, x, \alpha\right) .
\end{aligned}
$$

Therefore, we can put $K^{o}=S O\left(2 m_{b}\right) \times K_{2}^{\prime}=S O\left(2 k_{2}-2\right) \times K_{2}^{\prime}$ and

$$
\begin{aligned}
K_{2}^{\prime} & =\prod_{j=1}^{b-1} S O\left(2 m_{j}\right) \times P\left(\alpha_{1}, \cdots, \alpha_{a}, x, \alpha\right) \\
& \subset \prod_{j=1}^{b-1} S O\left(2 m_{j}+1\right) \times \prod_{i=1}^{a} S U\left(l_{i}+1\right) \times S U\left(k_{1}\right) \times T^{1} \\
& =G / S O\left(2 m_{b}+1\right)=G / S O\left(2 k_{2}-1\right) .
\end{aligned}
$$

By using $K_{2}^{o}=\operatorname{Spin}\left(2 k_{2}-1\right) \circ K_{2}^{\prime}$ (by $\left.(9.3)\right)$ and the above $K^{o}$, we have the following covering map $\varphi$ :

$$
\begin{array}{ccc}
\operatorname{Spin}\left(2 k_{2}-2\right) \times K_{2}^{\prime} & \longrightarrow & \operatorname{Spin}\left(2 k_{2}-1\right) \times K_{2}^{\prime} \\
\varphi \downarrow & & \downarrow \varphi \\
S O\left(2 k_{2}-2\right) \times K_{2}^{\prime} & \longrightarrow & K_{2}^{o}
\end{array}
$$


where the top and bottom maps are inclusions. Because the restricted representation $\left.\varphi\right|_{K_{2}^{\prime}}$ is the identity representation, there is the $K_{2}^{\prime}$ factor in $K_{2}^{o}$. Therefore, we have that

$$
\begin{aligned}
K_{2}^{o} & =S O\left(2 k_{2}-1\right) \times K_{2}^{\prime} \\
& =\prod_{j=1}^{b-1} S O\left(2 m_{j}\right) \times S O\left(2 k_{2}-1\right) \times P\left(\alpha_{1}, \cdots, \alpha_{a}, x, \alpha\right),
\end{aligned}
$$

and there is some inclusion $S O\left(2 k_{2}-1\right) \rightarrow K_{2}$ whose image is the factor $S O\left(2 m_{b}+\right.$ 1) $\subset G$. Because $K_{2}^{o} \subset K_{2} \subset G$, we can put $K_{2}$ is as follows:

$$
\begin{aligned}
& K_{2}=S O\left(2 k_{2}-1\right) \times K_{2}^{\prime \prime} \\
& \subset \quad G=S O\left(2 k_{2}-1\right) \times\left(\prod_{j=1}^{b-1} S O\left(2 m_{j}+1\right) \times \prod_{i=1}^{a} S U\left(l_{i}+1\right) \times S U\left(k_{1}\right) \times T^{1}\right),
\end{aligned}
$$

where $2 m_{b}+1=2 k_{2}-1$ and $K_{2}^{\prime \prime}$ is a subgroup of $\prod_{j=1}^{b-1} S O\left(2 m_{j}+1\right) \times \prod_{i=1}^{a} S U\left(l_{i}+\right.$ 1) $\times S U\left(k_{1}\right) \times T^{1}$ whose connected component is $K_{2}^{\prime}$. By using the argument of Section 8.2 , the $S O\left(2 k_{2}-1\right)$-factor in $K_{2}$ acts transitively on $K_{2} / K \cong S^{2 k_{2}-2}$. Therefore, for the natural projection $\Phi: K_{2}=S O\left(2 k_{2}-1\right) \times K_{2}^{\prime \prime} \rightarrow K_{2}^{\prime \prime}$, we have the following relation (see $[\mathbf{1 1}$, Lemma 8.0.2]):

$$
\Phi\left(K_{2}\right)=K_{2}^{\prime \prime}=\Phi(K) \text {. }
$$

Hence, we have

$$
K_{2}=S O\left(2 k_{2}-1\right) \times K_{2}^{\prime \prime}=S O\left(2 k_{2}-1\right) \times \Phi(K) \subset G .
$$

It follows that the inclusion $K_{2} \subset G$ is completely determined by $K$ (more precisely the projection $\Phi(K))$.

Next, we consider the slice representation $\sigma_{2}: K_{2} \rightarrow O\left(2 k_{2}-1\right)$. Since the $S O\left(2 k_{2}-1\right)$-factor in $K_{2}$ acts transitively on $K_{2} / K \cong S^{2 k_{2}-2}$, the restricted representation $\left.\sigma_{2}\right|_{S O\left(2 k_{2}-1\right)}$ is the natural isomorphism to $S O\left(2 k_{2}-1\right) \subset O\left(2 k_{2}-1\right)$. Hence, we have that

$$
\sigma_{2}\left(K_{2}^{\prime \prime}\right) \subset Z\left(S O\left(2 k_{2}-1\right)\right)=\left\{ \pm I_{2 k_{2}-1}\right\} \subset O\left(2 k_{2}-1\right) .
$$

Moreover, by (9.1), we have the following formula for $K$ :

$$
\begin{aligned}
& K=\sigma_{2}^{-1}\left(O\left(2 k_{2}-2\right)\right) \\
= & \left\{\left(A,\left(t_{1}, \cdots, t_{a}\right),\left(\begin{array}{cc}
X & 0 \\
0 & x
\end{array}\right), t\right) \mid \sigma_{1}(A) t_{1}^{\alpha_{1}} \cdots t_{a}^{\alpha_{a}} x t^{\alpha}=1\right\} \\
= & \left\{\left(\left(\begin{array}{cc}
A_{b} & 0 \\
0 & a_{b}
\end{array}\right), Y\right) \mid a_{b}=\operatorname{det} A_{b}^{-1}=\operatorname{det} \sigma_{2}(Y)\right\} . \\
\subset & S\left(O\left(2 k_{2}-2\right) \times O(1)\right) \times K_{2}^{\prime \prime} .
\end{aligned}
$$

Therefore, we can easily show that the following lemma by using (9.5).

LEMMA 9.2. The following two statements are equivalent:

(1): $\sigma_{2}\left(K_{2}^{\prime \prime}\right)=\left\{I_{2 k_{2}-1}\right\}$ (resp. $\left.\sigma_{2}\left(K_{2}^{\prime \prime}\right)=\left\{ \pm I_{2 k_{2}-1}\right\}\right)$;

(2): $K=S O\left(2 k_{2}-2\right) \times K_{2}^{\prime \prime}\left(\right.$ resp. $\left.r_{b}(K)=S\left(O\left(2 m_{b}\right) \times O(1)\right)\right)$.

Moreover, the following statement holds:

(3): if $\sigma_{2}\left(K_{2}^{\prime \prime}\right)=\left\{ \pm I_{2 k_{2}-1}\right\}$, then we have $K \neq S\left(O\left(2 m_{b}\right) \times O(1)\right) \times K_{2}^{\prime \prime}$. 
It follows from (9.5) and Lemma $9.2(1),(2)$ that the slice representation $\sigma_{2}$ : $K_{2} \rightarrow O\left(2 k_{2}-2\right)$ is also completely determined by $K$. Therefore, the tubular neighborhood $X_{2}=G \times_{K_{2}} D^{2 k_{2}-1}$ of $G / K_{2}$ is completely determined by $K$, and equivariantly diffeomorphic to the following manifold:

$$
\prod_{i=1}^{a} S^{2 l_{i}+1} \times_{T^{a}}\left(\left(\prod_{j=1}^{b-1} S^{2 m_{j}} \times D\left(\mathbb{R}^{2 k_{2}-1}\right)\right) \times_{\mathcal{A}} S\left(\mathbb{C}_{\mathfrak{c}}^{k_{1}}\right)\right)
$$

where $T^{a}$-quotient is defined similarly to that in the previous cases (e.g. the case (2)-(a)), $\mathcal{A} \simeq \mathcal{S}_{1} / \mathcal{S}_{1}^{o}$-quotient is defined by the following actions:

- on $\prod_{j=1}^{b-1} S^{2 m_{j}} \times D\left(\mathbb{R}^{2 k_{2}-1}\right)$ as the subgroup $\mathcal{A} \subset \prod_{j=1}^{b-1} \mathbb{Z}_{2} \times\left\{ \pm I_{2 k_{2}-1}\right\}$;

- on $S\left(\mathbb{C}_{\mathfrak{c}}^{k_{1}}\right) \subset \mathbb{C}_{\mathfrak{c}}^{k_{1}}$ by the representation $\sigma_{\mathbb{C}}: \mathcal{A} \rightarrow\{ \pm 1\}$ (induced by $\sigma_{1}$ ).

Moreover, by using (9.5) and Lemma $9.2(3)$, if $\left(1, \cdots, 1,-I_{2 k_{2}-1}\right) \in \mathcal{A} \subset$ $\prod_{j=1}^{b-1} \mathbb{Z}_{2} \times\left\{ \pm I_{2 k_{2}-1}\right\}$, then $\sigma_{\mathbb{C}}\left(1, \cdots, 1,-I_{2 k_{2}-1}\right)=-1$ (because if not so the principal isotropy subgroup is $\left.K=S\left(O\left(2 m_{b}\right) \times O(1)\right) \times K_{2}^{\prime \prime}\right)$. It follows that $\mathcal{A}$ acts on $\prod_{j=1}^{b-1} S^{2 m_{j}} \times D\left(\mathbb{R}^{2 k_{2}-1}\right) \times S\left(\mathbb{C}_{\mathfrak{c}}^{k_{1}}\right)$ freely; therefore, $X_{2}$ is a manifold.

By using Remark 4.2, we can easily check that the pair $(M, G)$ of the case (3)-(a) and $k_{2} \geq 3$ is as follows (up to essential isomorphism):

$$
\begin{aligned}
& M=\prod_{i=1}^{a} S^{2 l_{i}+1} \times_{T^{a}}\left(\prod_{j=1}^{b-1} S^{2 m_{j}} \times_{\mathcal{A}} S\left(\mathbb{R}^{2 k_{2}-1} \oplus \mathbb{C}_{\mathfrak{c}}^{k_{1}}\right)\right) ; \\
& G=\prod_{i=1}^{a} S U\left(l_{i}+1\right) \times \prod_{j=1}^{b-1} S O\left(2 m_{j}+1\right) \times S O\left(2 k_{2}-1\right) \times U\left(k_{1}\right),
\end{aligned}
$$

where $\mathcal{A}$ acts on $\prod_{j=1}^{b-1} S^{2 m_{j}} \times S\left(\mathbb{R}^{2 k_{2}-1} \oplus \mathbb{C}_{\mathfrak{c}}^{k_{1}}\right)$ as follows: on $\mathbb{C}_{\mathfrak{c}}^{k_{1}}$ by $\sigma_{\mathbb{C}}: \mathcal{A} \rightarrow$ $\{ \pm 1\}$; on $\prod_{j=1}^{b-1} S^{2 m_{j}} \times \mathbb{R}^{2 k_{2}-1}$ as the subgroup $\prod_{j=1}^{b-1} \mathbb{Z}_{2} \times\left\{ \pm I_{2 k_{2}-1}\right\}$ such that if $\left(1, \cdots, 1,-I_{2 k_{2}-1}\right) \in \mathcal{A}$ then $\sigma_{\mathbb{C}}\left(1, \cdots, 1,-I_{2 k_{2}-1}\right)=-1$.

This corresponds with the first manifold in Theorem 8.1 for $k_{2} \geq 3$.

9.2. The case $k_{2}=2$. Assume $k_{2}=2$. By (9.1) and (9.2), the covering of $K^{o}$ is as follows:

$$
\begin{aligned}
& \widetilde{K^{o}}=\operatorname{Spin}\left(2 k_{2}-2\right) \times \widetilde{K_{2}^{\prime}} \\
= & \prod_{j=1}^{b} \operatorname{Spin}\left(2 m_{j}\right) \times \prod_{i=1}^{a} S U\left(l_{i}\right) \times T^{a} \times S U\left(k_{1}-1\right) \times T^{1} \quad \text { for } k_{1} \geq 2 \quad \text { or } \\
= & \prod_{j=1}^{b} \operatorname{Spin}\left(2 m_{j}\right) \times \prod_{i=1}^{a} S U\left(l_{i}\right) \times T^{a} \quad \text { for } k_{1}=1
\end{aligned}
$$

where $l_{i} \geq 1$ for all $i=1, \cdots, a$. Comparing the above coverings of $K^{o}$ 's, there are the following three cases:

(i): $\operatorname{Spin}\left(2 k_{2}-2\right)=\operatorname{Spin}\left(2 m_{b}\right)$, and $k_{2}=m_{b}+1=2$;

(ii): $\operatorname{Spin}\left(2 k_{2}-2\right)=T_{a}$, where $T_{a}$ is the $a$-th factor of $T^{a}=T_{1} \times \cdots \times T_{a}$ $\left(T_{i} \simeq T^{1}\right)$

(iii): $\operatorname{Spin}\left(2 k_{2}-2\right)=T^{1}$, and $k_{1} \geq 2$. 
Remark 9.3. The above case (i) is the same as the case (i) in Section 9.1. By the same argument of Section 9.1 , we have that $(M, G)$ for the above case (i) is as follows $\left(k_{2}=2\right)$ :

$$
\begin{aligned}
& M=\prod_{i=1}^{a} S^{2 l_{i}+1} \times_{T^{a}}\left(\prod_{j=1}^{b-1} S^{2 m_{j}} \times_{\mathcal{A}} S\left(\mathbb{R}^{2 k_{2}-1} \oplus \mathbb{C}_{\mathfrak{c}}^{k_{1}}\right)\right) \\
& G=\prod_{i=1}^{a} S U\left(l_{i}+1\right) \times \prod_{j=1}^{b-1} S O\left(2 m_{j}+1\right) \times S O\left(2 k_{2}-1\right) \times U\left(k_{1}\right),
\end{aligned}
$$

where $T^{a}$ and $\mathcal{A}$ quotients are similarly defined as that of $M$ in Section 9.1. This corresponds with the first manifold in Theorem 8.1 for $k_{2}=2$. Hence, in this section, we may only discuss with the other cases: the case (ii) and (iii).

First we prove the following lemma.

LEMMA 9.4. In the above cases, the case (iii) does not occur.

Proof. By the definition of the case (iii),

$$
\widetilde{K_{2}^{\prime}}=\prod_{j=1}^{b} \operatorname{Spin}\left(2 m_{j}\right) \times \prod_{i=1}^{a} S U\left(l_{i}\right) \times T^{a} \times S U\left(k_{1}-1\right) .
$$

By $(9.2),(9.3)$, we can put $\widetilde{K_{2}^{o}}=\operatorname{Spin}\left(2 k_{2}-1\right) \times \widetilde{K_{2}^{\prime}}$. Therefore, with a method similar to that demonstrated in the proof of Lemma 9.1, there is the following commutative diagram $\left(k_{2}=2\right)$ :

$$
\begin{array}{ccc}
\operatorname{Spin}\left(2 k_{2}-2\right) \times \widetilde{K_{2}^{\prime}} & \stackrel{\widetilde{\iota}}{\longrightarrow} & \operatorname{Spin}\left(2 k_{2}-1\right) \times \widetilde{K_{2}^{\prime}} \\
\varphi \downarrow & & \downarrow \varphi \\
K^{o} & \stackrel{\iota}{\longrightarrow} & K_{2}^{o},
\end{array}
$$

where $\varphi$ is the finite covering, and $\widetilde{\iota}$ and $\iota$ are inclusion maps. Hence, the following sequence holds by the commutativity of the above diagram and the definition of $G$ (also see notations in Section 8.1):

$$
\begin{aligned}
q \circ \iota_{2} \circ \iota \circ \varphi(\operatorname{Spin}(2)) & =q \circ \iota_{2} \circ \varphi \circ \widetilde{\iota}(\operatorname{Spin}(2)) \\
& \subset q \circ \iota_{2} \circ \varphi(\operatorname{Spin}(3)) \\
& \subset q(G)=T^{1},
\end{aligned}
$$

On the other hand, by (9.1), we have

$$
q \circ \iota_{2} \circ \iota \circ \varphi(\operatorname{Spin}(2))=T^{1}=q(G)=q \circ \iota_{2} \circ \iota\left(K^{o}\right) .
$$

Consequently, we have $q \circ \iota_{2} \circ \phi(\operatorname{Spin}(3))=T^{1}$. However, this gives a contradiction, because there is no non-trivial representation from $\operatorname{Spin}(3)$ to $T^{1}$. It follows that the case (iii) does not occur. tion.

By Remark 9.3 and Lemma 9.4, we may only study the case (ii) in this subsec-

Assume the case (ii) occurs, that is, $\operatorname{Spin}\left(2 k_{2}-2\right)=T_{a}$, where $T_{a}$ is the $a$-th factor of $T^{a}=T_{1} \times \cdots \times T_{a}\left(T_{i} \simeq T^{1}\right)$. Let $\pi_{j}: \operatorname{Spin}\left(2 m_{j}\right) \rightarrow S O\left(2 m_{j}\right)$ be the double covering. In order to study this case, we divide this case into two parts:

- the case $k_{1}=1$;

- the case $k_{1} \geq 2$. 
9.2.1. The case $k_{1}=1$. First, we assume $k_{1}=1$. Then, we have the following finite covering:

$$
\pi: \prod_{j=1}^{b} \operatorname{Spin}\left(2 m_{j}\right) \times \prod_{i=1}^{a} S U\left(l_{i}\right) \times T^{a}=\widetilde{K_{2}^{\prime}} \times T_{a} \longrightarrow K^{o}
$$

such that

$$
\begin{aligned}
\pi\left(A_{j}, B_{i}, t_{i}\right) & =\left(\pi_{j}\left(A_{j}\right),\left(\begin{array}{cc}
B_{i} t_{i}^{-1 / l_{i}} & 0 \\
0 & t_{i}
\end{array}\right), t_{1}^{-\alpha_{1}} \cdots t_{a}^{-\alpha_{a}}\right) \\
& \in K^{o} \subset \prod_{j=1}^{b} S O\left(2 m_{j}\right) \times \prod_{i=1}^{a} S\left(U\left(l_{i}\right) \times U(1)\right) \times T^{1},
\end{aligned}
$$

for the element $\left(A_{j}, B_{i}, t_{i}\right) \in \prod_{j=1}^{b} \operatorname{Spin}\left(2 m_{j}\right) \times \prod_{i=1}^{a} S U\left(l_{i}\right) \times T^{a}$.

If $\alpha_{a} \neq 0$, then $K^{o}$ has the following subgroup:

$$
\begin{aligned}
& \pi\left(T_{a}\right)=\pi\left(\operatorname{Spin}\left(2 k_{2}-2\right)\right)=\left\{\left(\left(\begin{array}{cc}
I_{l_{a}} t_{a}^{-1 / l_{a}} & 0 \\
0 & t_{a}
\end{array}\right), t_{a}^{-\alpha_{a}}\right) \mid t_{a} \in T_{a}\right\} \\
& \subset \quad\left(S\left(U\left(l_{a}\right) \times U(1)\right) \times T^{1}\right) \cap K^{o},
\end{aligned}
$$

where $I_{l_{a}}$ is the identity element in $U\left(l_{a}\right)$. Therefore, for the following commutative diagram (by (9.2) and (9.3)):

$$
\begin{array}{ccc}
T_{a} \times \widetilde{K_{2}^{\prime}} & \stackrel{\tau}{\longrightarrow} & \operatorname{Spin}(3) \times \widetilde{K_{2}^{\prime}} \\
\pi \downarrow & & \downarrow \widetilde{\pi} \\
K^{o} & \stackrel{\iota}{\longrightarrow} & K_{2}^{o},
\end{array}
$$

we have the following sequence (also see notations in Section 8.1):

$$
\begin{aligned}
T^{1}=q \circ \iota_{2} \circ \iota \circ \pi\left(T_{a}\right) & =q \circ \iota_{2} \circ \iota \circ \pi\left(\operatorname{Spin}\left(2 k_{2}-2\right)\right) \\
& =q \circ \iota_{2} \circ \tilde{\pi} \circ \widetilde{\iota}\left(\operatorname{Spin}\left(2 k_{2}-2\right)\right) \\
& \subset q \circ \iota_{2} \circ \tilde{\pi}\left(\operatorname{Spin}\left(2 k_{2}-1\right)\right) \\
& \subset q(G)=T^{1} .
\end{aligned}
$$

This gives a contradiction with a method similar to that demonstrated in the proof of Lemma 9.4. Hence, we have $\alpha_{a}=0$.

Because $\alpha_{a}=0$, we have that $\pi\left(T_{a} \times S U\left(l_{a}\right)\right)=S\left(U\left(l_{a}\right) \times U(1)\right)$. Therefore, there is the following sequence:

$$
\begin{aligned}
S\left(U\left(l_{a}\right) \times U(1)\right) & =p_{a} \circ \iota_{2} \circ \iota \circ \pi\left(T_{a} \times S U\left(l_{a}\right)\right) \\
& =p_{a} \circ \iota_{2} \circ \tilde{\pi} \circ \widetilde{\iota}\left(T_{a} \times S U\left(l_{a}\right)\right) \\
& \subset p_{a} \circ \iota_{2} \circ \tilde{\pi}\left(\operatorname{Spin}(3) \times S U\left(l_{a}\right)\right) \\
& \subset p_{a}(G)=S U\left(l_{a}+1\right) .
\end{aligned}
$$

Because $\operatorname{Spin}(3)$ and $S U\left(l_{a}\right)$ are simple and $\operatorname{dim}\left(S U\left(l_{a}\right) \times \operatorname{Spin}(3)\right)=l_{a}^{2}+2$ and $\operatorname{dim} S\left(U\left(l_{a}\right) \times U(1)\right)=l_{a}^{2}$, we have that $p_{a} \circ \iota_{2} \circ \tilde{\pi}\left(S U\left(l_{a}\right) \times \operatorname{Spin}(3)\right) \neq S\left(U\left(l_{a}\right) \times\right.$ $U(1))$. Since $S\left(U\left(l_{a}\right) \times U(1)\right)$ is a maximal rank, maximal subgroup in $S U\left(l_{a}+1\right)$ (see $[\mathbf{1 5}]$ ), we have

$$
p_{a} \circ \iota_{2} \circ \widetilde{\pi}\left(S U\left(l_{a}\right) \times \operatorname{Spin}(3)\right)=S U\left(l_{a}+1\right) .
$$


Comparing their dimension $\left(\operatorname{dim}\left(S U\left(l_{a}\right) \times \operatorname{Spin}(3)\right)=l_{a}^{2}+2\right.$ and $\operatorname{dim} S U\left(l_{a}+1\right)=$ $l_{a}^{2}+2 l_{a}$ ), we have

$$
l_{a}=1
$$

$($ remark $\operatorname{Spin}(3) \simeq S U(2))$. Hence, in the case $k_{1}=1$, we can regard

$$
\begin{aligned}
& \left(S U\left(l_{a}+1\right), S\left(U\left(l_{a}\right) \times U(1)\right)\right) \\
= & \left(\operatorname{Spin}\left(2 k_{2}-1\right), \operatorname{Spin}\left(2 k_{2}-2\right)\right) \\
= & \left(\operatorname{Spin}\left(2 m_{b+1}+1\right), \operatorname{Spin}\left(2 m_{b+1}\right)\right)
\end{aligned}
$$

where $m_{b+1}=1$. Therefore, by regarding $b+1$ as $b$ and $a-1$ as $a$, we can regard the case (ii) with $k_{1}=1$ as the case (i). Hence, this case corresponds with the first case of Theorem 8.1 which satisfies $k_{1}=1$ and $k_{2}=2$ by using Remark 9.3 .

9.2.2. The case $k_{1} \geq 2$. Next, we study the other case: the case $k_{1} \geq 2$.

If $k_{1} \geq 2$, then we have the following finite coverings:

$$
\pi: \prod_{j=1}^{b} \operatorname{Spin}\left(2 m_{j}\right) \times \prod_{i=1}^{a} S U\left(l_{i}\right) \times T^{a} \times S U\left(k_{1}-1\right) \times T^{1} \rightarrow K^{o}
$$

such that,

$$
\begin{aligned}
& \pi\left(A_{j}, B_{i}, t_{i}, Y, t\right)=\left(\pi_{j}\left(A_{j}\right),\left(\begin{array}{cc}
B_{i} t_{i}^{-1 / l_{i}} & 0 \\
0 & t_{i}
\end{array}\right),\left(\begin{array}{cc}
Y x^{-1 /\left(k_{1}-1\right)} & 0 \\
0 & x
\end{array}\right), t\right) \\
\in & \prod_{j=1}^{b} S O\left(2 m_{j}\right) \times \prod_{i=1}^{a} S\left(U\left(l_{i} \times U(1)\right) \times S\left(U\left(k_{1}-1\right) \times U(1)\right) \times T^{1},\right.
\end{aligned}
$$

where $x=t_{1}^{-\alpha_{1}} \cdots t_{a}^{-\alpha_{a}} t^{-\alpha}$.

If $\alpha_{a}=0$, then we can easily show that $l_{a}=1$ and this case corresponds with the first case of Theorem 8.1 which satisfies $k_{1} \geq 2$ and $k_{2}=2$ with the method similar to that demonstrated in the previous section (Section 9.2.1). Therefore, we may only discuss with the case $\alpha_{a} \neq 0$.

Assume $\alpha_{a} \neq 0$. We will prove this case does not occur. First, we prove the following lemma.

Lemma 9.5. If $k_{1} \geq 2$ and $\alpha_{a} \neq 0$, then we can put $l_{a}=1, k_{1}=2$ and $\alpha_{a}= \pm 1$.

Proof. If $k_{1} \geq 2$ and $\alpha_{a} \neq 0$, then $K^{o}$ has the following subgroups $\left(k_{2}=2\right)$ :

$$
\begin{aligned}
& \pi\left(S U\left(l_{a}\right) \times \operatorname{Spin}\left(2 k_{2}-2\right)\right) \\
& =\left\{\left(\left(\begin{array}{cc}
B_{a} t_{a}^{-1 / l_{a}} & 0 \\
0 & t_{a}
\end{array}\right),\left(\begin{array}{cc}
I_{k_{1}-1} t_{a}^{\alpha_{a} /\left(k_{1}-1\right)} & 0 \\
0 & t_{a}^{-\alpha_{a}}
\end{array}\right)\right)\right\} \\
& \pi\left(\operatorname{Spin}\left(2 k_{2}-2\right) \times S U\left(k_{1}-1\right)\right) \\
& =\left\{\left(\left(\begin{array}{cc}
I_{l_{a}} t_{a}^{-1 / l_{a}} & 0 \\
0 & t_{a}
\end{array}\right),\left(\begin{array}{cc}
Y t_{a}^{\alpha_{a} /\left(k_{1}-1\right)} & 0 \\
0 & t_{a}^{-\alpha_{a}}
\end{array}\right)\right)\right\}
\end{aligned}
$$

where $t_{a} \in T_{a}=\operatorname{Spin}\left(2 k_{2}-2\right), B_{a} \in S U\left(l_{a}\right), Y \in S U\left(k_{1}-1\right)$, and $I_{k_{1}-1}, I_{l_{a}}$ are identity elements in $U\left(k_{1}-1\right)$ and $U\left(l_{a}\right)$, respectively. Moreover, there is the 
following commutative diagram by (9.2) and (9.3):

$$
\begin{array}{ccc}
\left(S U\left(l_{a}\right) \times \operatorname{Spin}(2) \times S U\left(k_{1}-1\right)\right) \times \widetilde{K_{2}^{\prime \prime}} & \stackrel{\tau}{\longrightarrow} & \left(S U\left(l_{a}\right) \times S p i n(3) \times S U\left(k_{1}-1\right)\right) \times \widetilde{K_{2}^{\prime \prime}} \\
K^{o} & \stackrel{\iota}{\longrightarrow} & \downarrow \widetilde{\pi} \\
& & K_{2}^{o},
\end{array}
$$

where $\widetilde{K_{2}^{\prime \prime}}=\prod_{j=1}^{b} \operatorname{Spin}\left(2 m_{j}\right) \times \prod_{i=1}^{a-1} S U\left(l_{i}\right) \times T^{a-1} \times T^{1}$. Then, we have the following sequence by $(9.6)$ :

$$
\begin{aligned}
S\left(U\left(l_{a}\right) \times U(1)\right) & =p_{a} \circ \iota_{2} \circ \iota \circ \pi\left(S U\left(l_{a}\right) \times \operatorname{Spin}(2)\right) \\
& =p_{a} \circ \iota_{2} \circ \widetilde{\pi} \circ \widetilde{\iota}\left(S U\left(l_{a}\right) \times \operatorname{Spin}(2)\right) \\
& \subset p_{a} \circ \iota_{2} \circ \widetilde{\pi}\left(S U\left(l_{a}\right) \times \operatorname{Spin}(3)\right) \\
& \subset p_{a}(G)=S U\left(l_{a}+1\right) .
\end{aligned}
$$

With a method similar to that demonstrated in the proof of $l_{a}=1$ in Section 9.2.1, we have $l_{a}=1$. Moreover, by (9.7), we have the following sequence:

$$
\begin{aligned}
S\left(U\left(k_{1}-1\right) \times U(1)\right) & =p \circ \iota_{2} \circ \iota \circ \pi\left(\operatorname{Spin}(2) \times S U\left(k_{1}-1\right)\right) \\
& =p \circ \iota_{2} \circ \widetilde{\pi} \circ \widetilde{\iota}\left(\operatorname{Spin}(2) \times S U\left(k_{1}-1\right)\right) \\
& \subset p \circ \iota_{2} \circ \widetilde{\pi}\left(\operatorname{Spin}(3) \times S U\left(k_{1}-1\right)\right) \\
& \subset p(G)=S U\left(k_{1}\right) .
\end{aligned}
$$

Similarly, we have $k_{1}=2$

Moreover, we can easily show that $p \circ \iota_{2} \circ \tilde{\pi}: \operatorname{Spin}(3) \rightarrow S U\left(k_{1}\right)=S U(2)$ is an isomorphic map. By considering the restricted representation to $\operatorname{Spin}(2)$ of this isomorphic map and using (9.7), we also have $\alpha_{a}= \pm 1$.

By Lemma 9.5, we have $l_{a}=1, k_{1}=2$ and $\left|\alpha_{a}\right|=1$; moreover, we have the following commutative diagram (see the proof of Lemma 9.5):

$$
\begin{array}{ccc}
\operatorname{Spin}(2) \times \widetilde{K_{2}^{\prime \prime}} & \stackrel{\widetilde{\iota}}{\longrightarrow} & \operatorname{Spin}(3) \times \widetilde{K_{2}^{\prime \prime}} \\
\pi \downarrow & & \downarrow \widetilde{\pi} \\
K^{o} & \stackrel{\iota}{\longrightarrow} & K_{2}^{o} .
\end{array}
$$

Then, we have the following sequence:

$$
\begin{aligned}
H & =\left(p_{a} \times p\right) \circ \iota_{2} \circ \iota \circ \pi(\operatorname{Spin}(2)) \\
& =\left(p_{a} \times p\right) \circ \iota_{2} \circ \widetilde{\pi} \circ \widetilde{\imath}(\operatorname{Spin}(2)) \\
& \subset\left(p_{a} \times p\right) \circ \iota_{2} \circ \widetilde{\pi}(\operatorname{Spin}(3)) \\
& \subset p_{a} \times p(G)=S U\left(l_{a}+1\right) \times S U\left(k_{1}\right)=S U(2) \times S U(2) .
\end{aligned}
$$

Here, $H$ is one of the followings, because of (9.6) and (9.7) in the proof of Lemma 9.5 and $\left|\alpha_{a}\right|=1$ :

$$
\begin{aligned}
& \Delta=\left\{\left(\begin{array}{cc}
t_{a}^{-1} & 0 \\
0 & t_{a}
\end{array}\right),\left(\begin{array}{cc}
t_{a}^{-1} & 0 \\
0 & t_{a}
\end{array}\right)\right\} \subset S U(2) \times S U(2) \text { for } \alpha_{a}=-1 ; \\
& \Delta^{\prime}=\left\{\left(\begin{array}{cc}
t_{a}^{-1} & 0 \\
0 & t_{a}
\end{array}\right),\left(\begin{array}{cc}
t_{a} & 0 \\
0 & t_{a}^{-1}
\end{array}\right)\right\} \subset S U(2) \times S U(2) \text { for } \alpha_{a}=1 .
\end{aligned}
$$

Since $\Delta$ and $\Delta^{\prime}$ are conjugate in $G$, we can take $\alpha_{a}=-1$ and $H=\Delta$. Because $\operatorname{Spin}(3) \simeq S U(2)$ and $H=\Delta$, we can easily show that

$$
\left(p_{a} \times p\right) \circ \iota_{2} \circ \tilde{\pi}(\operatorname{Spin}(3))=\{(X, X) \mid X \in S U(2)\} \subset S U(2) \times S U(2),
$$


i.e., the diagonal subgroup. By the definition of $\pi$ and the above argument, we see the followings:

$$
\begin{aligned}
& \iota \circ \pi\left(\operatorname{Spin}(2) \times T^{1}\right) \\
= & \left\{\left(\left(\begin{array}{cc}
t_{a}^{-1} & 0 \\
0 & t_{a}
\end{array}\right),\left(\begin{array}{cc}
t_{a}^{-1} t^{\alpha} & 0 \\
0 & t_{a} t^{-\alpha}
\end{array}\right), t\right) \in S U(2) \times S U(2) \times T^{1}\right\} \\
\subset & \tilde{\pi}\left(\operatorname{Spin}(3) \times T^{1}\right) \\
= & \left\{(X, X, t) \in S U(2) \times S U(2) \times T^{1}\right\} .
\end{aligned}
$$

It follows that $\alpha=0$. However, this gives a contradiction because $\alpha \in \mathbb{N}$ (see Section 4.1.1). Therefore, this case (the case $k_{1} \geq 2$ and $\alpha_{a} \neq 0$ ) does not occur.

\section{The case (3)-(b)}

In this section, we study the case (3)-(b). From Section 4.1, we have

$$
\begin{aligned}
G= & \prod_{j=1}^{b} S O\left(2 m_{j}+1\right) \times \prod_{i=1}^{a} S U\left(l_{i}+1\right) \times S O\left(2 k_{1}\right), \\
K_{1}= & \mathcal{S}_{1} \times \prod_{i=1}^{a} S\left(U\left(l_{i}\right) \times U(1)\right) \times S O\left(2 k_{1}\right), \\
(10.1) K= & \prod_{i=1}^{a} S\left(U\left(l_{i}\right) \times U(1)\right) \\
& \times\left\{\left(A,\left(\begin{array}{cc}
X & 0 \\
0 & x
\end{array}\right)\right) \in \mathcal{S}_{1} \times S\left(O\left(2 k_{1}-1\right) \times O(1)\right) \mid \sigma_{1}(A) x=1\right\},
\end{aligned}
$$

where $k_{1} \geq 2$. From Lemma 8.2, we also have

$$
\begin{aligned}
& K^{o}=\operatorname{Spin}\left(2 k_{2}-2\right) \circ K_{2}^{\prime}, \\
& K_{2}^{o}=\operatorname{Spin}\left(2 k_{2}-1\right) \circ K_{2}^{\prime}
\end{aligned}
$$

Therefore, we have the covering of $K^{o}$ as follows by (10.1) and (10.2):

$$
\begin{aligned}
& \widetilde{K^{o}}=\operatorname{Spin}\left(2 k_{2}-2\right) \times \widetilde{K_{2}^{\prime}} \\
= & \prod_{j=1}^{b} \operatorname{Spin}\left(2 m_{j}\right) \times \prod_{i=1}^{a} S U\left(l_{i}\right) \times T^{a} \times \operatorname{Spin}\left(2 k_{1}-1\right) .
\end{aligned}
$$

Comparing the above covering of $K^{o}$ 's and using the fact that $\operatorname{Spin}(2) \simeq T^{1}$, $\operatorname{Spin}(3) \simeq S U(2), \operatorname{Spin}(4) \simeq S U(2) \times S U(2)$ and $\operatorname{Spin}(6) \simeq S U(4)$, there are the following five cases:

(i): $\operatorname{Spin}\left(2 k_{2}-2\right)=\operatorname{Spin}\left(2 m_{b}\right)$ and $k_{2}=m_{b}+1 \geq 2$;

(ii): $\operatorname{Spin}\left(2 k_{2}-2\right)=T_{a}$ and $k_{2}=2$, where $T_{a}$ is the $a$-th factor of $T^{a}=$ $T_{1} \times \cdots \times T_{a}\left(T_{i} \simeq T^{1}\right)$

(iii): $\operatorname{Spin}\left(2 k_{2}-2\right)=S U\left(l_{a-1}\right) \times S U\left(l_{a}\right)$ and $l_{a}=l_{a-1}=2, k_{2}=3$;

(iv): $\operatorname{Spin}\left(2 k_{2}-2\right)=S U\left(l_{a}\right) \times \operatorname{Spin}\left(2 k_{1}-1\right)$ and $l_{a}=2, k_{1}=2, k_{2}=3$;

(v): $\operatorname{Spin}\left(2 k_{2}-2\right)=S U\left(l_{a}\right)$ and $l_{a}=4, k_{2}=4$.

Similarly to Lemma 9.1, we can show the following lemma.

LEMMA 10.1. In the above cases, the cases from (iii) to (v) do not occur. 
Proof. For the cases (iii) and (iv), we can apply a method similar to the proof of that the case (ii) does not occur in Lemma 9.1. For the case (v), we can apply a method similar to the proof of that the case (iv) does not occur in Lemma 9.1. So we may omit the detail of the proof.

From the next section, we study the cases (i) and (ii): we call them (3)-(b)-(i) and (3)-(b)-(ii), respectively.

10.1. The case (3)-(b)-(i). Suppose the case (3)-(b)-(i) occurs, that is,

$$
\operatorname{Spin}\left(2 k_{2}-2\right)=\operatorname{Spin}\left(2 m_{b}\right) \quad \text { and } \quad k_{2}=m_{b}+1 \geq 2 .
$$

It follows from (10.1) and (10.2) that the following relation holds:

$$
\begin{aligned}
\operatorname{Spin}\left(2 k_{2}-2\right) \circ K_{2}^{\prime} & =\operatorname{Spin}\left(2 m_{b}\right) \circ K_{2}^{\prime} \\
& =\prod_{j=1}^{b} S O\left(2 m_{j}\right) \times \prod_{i=1}^{a} S\left(U\left(l_{i}\right) \times U(1)\right) \times S O\left(2 k_{1}-1\right)=K^{o} .
\end{aligned}
$$

Therefore, we can put $K^{o}=S O\left(2 m_{b}\right) \times K_{2}^{\prime}=S O\left(2 k_{2}-2\right) \times K_{2}^{\prime}$ and

$$
K_{2}^{\prime}=\prod_{j=1}^{b-1} S O\left(2 m_{j}\right) \times \prod_{i=1}^{a} S\left(U\left(l_{i}\right) \times U(1)\right) \times S O\left(2 k_{1}-1\right) .
$$

Because $K_{2}^{o}=\operatorname{Spin}\left(2 k_{2}-1\right) \circ K_{2}^{\prime}$ (by (10.3)), by using the same argument in Section 9.1 , we have

$$
K_{2}^{o}=\prod_{i=1}^{a} S\left(U\left(l_{i}\right) \times U(1)\right) \times \prod_{j=1}^{b-1} S O\left(2 m_{b}\right) \times S O\left(2 k_{2}-1\right) \times S O\left(2 k_{1}-1\right),
$$

and there is the inclusion $S O\left(2 k_{2}-1\right) \rightarrow K_{2}$ such that its image is $S O\left(2 m_{b}+1\right) \subset G$. Because $K_{2}^{o} \subset K_{2} \subset G$, we can put $K_{2}$ is as follows:

$$
\begin{aligned}
& K_{2}=S O\left(2 k_{2}-1\right) \times K_{2}^{\prime \prime} \\
& \subset G=S O\left(2 k_{2}-1\right) \times\left(\prod_{j=1}^{b-1} S O\left(2 m_{j}+1\right) \times \prod_{i=1}^{a} S U\left(l_{i}+1\right) \times S O\left(2 k_{1}\right)\right),
\end{aligned}
$$

where $2 m_{b}+1=2 k_{2}-1$ and $K_{2}^{\prime \prime}$ is a subgroup of $\prod_{j=1}^{b-1} S O\left(2 m_{j}+1\right) \times \prod_{i=1}^{a} S U\left(l_{i}+\right.$ 1) $\times S O\left(2 k_{1}\right)$. By using the same argument of Section 9.1 , we have that

$$
K_{2}=S O\left(2 k_{2}-1\right) \times \Phi(K) \subset G \text { such that } \Phi(K)=\Phi\left(K_{2}\right)=K_{2}^{\prime \prime},
$$

where $\Phi: G \rightarrow G / S O\left(2 k_{2}-1\right)=G / S O\left(2 m_{b}+1\right)$ is the natural projection. It follows that the inclusion $K_{2} \subset G$ is completely determined by $K$.

Next, we consider the slice representation $\sigma_{2}: K_{2} \rightarrow O\left(2 k_{2}-1\right)$. By the same reason demonstrated in Section 9.1, the restricted slice representation $\left.\sigma_{2}\right|_{S O\left(2 k_{2}-1\right)}$ : $S O\left(2 k_{2}-1\right) \rightarrow O\left(2 k_{2}-1\right)$ is the natural inclusion. Hence, we have that

$$
\sigma_{2}\left(K_{2}^{\prime \prime}\right) \subset Z\left(S O\left(2 k_{2}-1\right)\right)=\left\{ \pm I_{2 k_{2}-1}\right\} \subset O\left(2 k_{2}-1\right) .
$$


Moreover, by (10.1), we have the following formula for $K$ :

$$
\begin{aligned}
& K=\sigma_{2}^{-1}\left(O\left(2 k_{2}-2\right)\right) \\
= & \left\{\left(\left(\begin{array}{cc}
B & 0 \\
0 & b
\end{array}\right), Y\right) \in S\left(O\left(2 k_{2}-2\right) \times O(1)\right) \times K_{2}^{\prime \prime} \mid b=\operatorname{det} B^{-1}=\operatorname{det} \sigma_{2}(Y)\right\} \\
= & \prod_{i=1}^{a} S\left(U\left(l_{i}\right) \times U(1)\right) \times\left\{\left(A,\left(\begin{array}{cc}
X & 0 \\
0 & x
\end{array}\right)\right) \in \mathcal{S}_{1} \times S\left(O\left(2 k_{1}-1\right) \times O(1)\right) \mid \sigma_{1}(A) x=1\right\} .
\end{aligned}
$$

Therefore, we can easily show that the following lemma by using the above formula for $K$.

LEMMA 10.2. The following two statements are equivalent:

(1): $\sigma_{2}\left(K_{2}^{\prime \prime}\right)=\left\{I_{2 k_{2}-1}\right\}$ (resp. $\left.\sigma_{2}\left(K_{2}^{\prime \prime}\right)=\left\{ \pm I_{2 k_{2}-1}\right\}\right)$;

(2): $K=S O\left(2 k_{2}-2\right) \times K_{2}^{\prime \prime}\left(\right.$ resp. $\left.r_{b}(K)=S\left(O\left(2 m_{b}\right) \times O(1)\right)\right)$.

Moreover, the following statement holds:

(3): if $\sigma_{2}\left(K_{2}^{\prime \prime}\right)=\left\{ \pm I_{2 k_{2}-1}\right\}$ then $K \neq S\left(O\left(2 m_{b}\right) \times O(1)\right) \times K_{2}^{\prime \prime}$.

It follows from this Lemma 10.2 and the above formula for $K$ that the slice representation $\sigma_{2}: K_{2} \rightarrow O\left(2 k_{2}-2\right)$ is also completely determined by $K$. Therefore, the tubular neighborhood of $G / K_{2}$ is completely determined by $K$ and equivariantly diffeomorphic to the following manifold:

$$
\prod_{i=1}^{a} \mathbb{C} P\left(l_{i}\right) \times\left(\prod_{j=1}^{b-1} S^{2 m_{j}} \times D\left(\mathbb{R}^{2 k_{2}-1}\right)\right) \times_{\mathcal{A}} S\left(\mathbb{R}^{2 k_{1}}\right),
$$

where $\mathcal{A} \simeq \mathcal{S}_{1} / \mathcal{S}_{1}^{o}$-quotient is defined by the following actions: on $\prod_{j=1}^{b-1} S^{2 m_{j}} \times$ $D\left(\mathbb{R}^{2 k_{2}-1}\right)$ as the subgroup $\mathcal{A} \subset \prod_{j=1}^{b-1} \mathbb{Z}_{2} \times\left\{ \pm I_{2 k_{2}-1}\right\}$; and on $S\left(\mathbb{R}^{2 k_{1}}\right) \subset \mathbb{R}^{2 k_{1}}$ by the representation $\sigma_{\mathbb{R}}: \mathcal{A} \rightarrow\left\{ \pm I_{2 k_{1}}\right\}$ (induced by $\sigma_{1}$ ). Moreover, by using Lemma $10.2(3)$, if $\left(1, \cdots, 1,-I_{2 k_{2}-1}\right) \in \mathcal{A} \subset \prod_{j=1}^{b-1} \mathbb{Z}_{2} \times\left\{ \pm I_{2 k_{2}-1}\right\}$, then $\sigma_{\mathbb{R}}\left(1, \cdots, 1,-I_{2 k_{2}-1}\right)=-I_{2 k_{1}}$. It follows that $\mathcal{A}$ acts on $\prod_{j=1}^{b-1} S^{2 m_{j}} \times D\left(\mathbb{R}^{2 k_{2}-1}\right) \times$ $S\left(\mathbb{R}^{2 k_{1}}\right)$ freely; therefore, $X_{2}$ is a manifold.

From Remark 4.2, we can easily check that the pair of $(M, G)$ of the case (3)-(b)-(i) is as follows:

$$
\begin{aligned}
& M=\prod_{i=1}^{a} \mathbb{C} P\left(l_{i}\right) \times \prod_{j=1}^{b-1} S^{2 m_{j}} \times_{\mathcal{A}} S\left(\mathbb{R}^{2 k_{2}-1} \oplus \mathbb{R}^{2 k_{1}}\right) ; \\
& G=\prod_{i=1}^{a} S U\left(l_{i}+1\right) \times \prod_{j=1}^{b-1} S O\left(2 m_{j}+1\right) \times S O\left(2 k_{2}-1\right) \times S O\left(2 k_{1}\right),
\end{aligned}
$$

where $\mathcal{A}$ acts on $\prod_{j=1}^{b-1} S^{2 m_{j}} \times S\left(\mathbb{R}^{2 k_{2}-1} \oplus \mathbb{R}^{2 k_{1}}\right)$ as follows:

- on $\mathbb{R}^{2 k_{1}}$ by $\sigma_{\mathbb{R}}: \mathcal{A} \rightarrow\left\{ \pm I_{2 k_{1}}\right\}$;

- on $\prod_{j=1}^{b-1} S^{2 m_{j}} \times \mathbb{R}^{2 k_{2}-1}$ as a subgroup $\prod_{j=1}^{b-1} \mathbb{Z}_{2} \times\left\{ \pm I_{2 k_{2}-1}\right\}$ such that if $\left(1, \cdots, 1,-I_{2 k_{2}-1}\right) \in \mathcal{A}$, then $\sigma_{\mathbb{R}}\left(1, \cdots, 1,-I_{2 k_{2}-1}\right)=-I_{2 k_{1}}$.

This corresponds with the second manifold in Theorem 8.1. 
10.2. The case (3)-(b)-(ii). Suppose the case (3)-(b)-(ii) occurs, that is,

$$
\operatorname{Spin}\left(2 k_{2}-2\right)=T_{a} \quad \text { and } \quad k_{2}=2,
$$

where $T_{a}$ is the $a$-th factor of $T^{a}=T_{1} \times \cdots \times T_{a}\left(T_{i} \simeq T^{1}\right)$. Let $\psi: \widetilde{K^{o}} \rightarrow K^{o}$ be the finite covering projection, where $\widetilde{K^{o}}$ is a product of Lie groups. By (10.1), we can easily show that

$$
\psi\left(\operatorname{Spin}\left(2 k_{2}-2\right)\right)=\left\{\left(\begin{array}{cc}
I_{l_{a}} t_{a}^{-1 / l_{a}} & 0 \\
0 & t_{a}
\end{array}\right) \mid t_{a} \in T_{a}=\operatorname{Spin}\left(2 k_{2}-2\right)\right\} .
$$

Moreover, there is the following commutative diagram by (10.2) and (10.3):

$$
\begin{array}{ccc}
\left(S U\left(l_{a}\right) \times \operatorname{Spin}\left(2 k_{2}-2\right)\right) \times \widetilde{K_{2}^{\prime \prime}} & \stackrel{\widetilde{\iota}}{\longrightarrow} & \left(S U\left(l_{a}\right) \times S p i n\left(2 k_{2}-1\right)\right) \times \widetilde{K_{2}^{\prime \prime}} \\
\psi \downarrow & & \downarrow \widetilde{\psi} \\
K^{o} & \stackrel{\iota}{\longrightarrow} & K_{2}^{o},
\end{array}
$$

where $\widetilde{K_{2}^{\prime \prime}}=\prod_{j=1}^{b} \operatorname{Spin}\left(2 m_{j}\right) \times \prod_{i=1}^{a-1} S U\left(l_{i}\right) \times T^{a-1} \times \operatorname{Spin}\left(2 k_{1}-1\right)$. Because $p_{a}\left(K^{o}\right)=S\left(U\left(l_{a}\right) \times U(1)\right)$ (by $\left.(10.1)\right)$, we have the following sequence $\left(k_{2}=2\right)$ :

$$
\begin{aligned}
& S\left(U\left(l_{a}\right) \times U(1)\right) \\
= & p_{a} \circ \iota_{2} \circ \iota \circ \psi\left(S U\left(l_{a}\right) \times \operatorname{Spin}\left(2 k_{2}-2\right)\right) \\
= & p_{a} \circ \iota_{2} \circ \widetilde{\psi} \circ \widetilde{\iota}\left(S U\left(l_{a}\right) \times \operatorname{Spin}\left(2 k_{2}-2\right)\right) \\
\subset & p_{a} \circ \iota_{2} \circ \widetilde{\psi}\left(S U\left(l_{a}\right) \times \operatorname{Spin}\left(2 k_{2}-1\right)\right) \\
\subset & p_{a}(G)=S U\left(l_{a}+1\right) .
\end{aligned}
$$

Therefore, with a method similar to the proof of $l_{a}=1$ in Section 9.2.1, we also have $l_{a}=1$ (remark $\left.\operatorname{Spin}(3) \simeq S U(2)\right)$. Hence, we have

$$
\begin{aligned}
K_{2}^{o} & =\prod_{j=1}^{b} S O\left(2 m_{b}\right) \times \prod_{i=1}^{a-1} S\left(U\left(l_{i}\right) \times U(1)\right) \times S U(2) \times S O\left(2 k_{1}-1\right) \\
& =\prod_{j=1}^{b} S O\left(2 m_{b}\right) \times \prod_{i=1}^{a-1} S\left(U\left(l_{i}\right) \times U(1)\right) \times \operatorname{Spin}(3) \times S O\left(2 k_{1}-1\right) .
\end{aligned}
$$

By the similar argument of Section 10.1 and using (10.1), we have that

$$
\begin{aligned}
K_{2}= & \prod_{i=1}^{a-1} S\left(U\left(l_{i}\right) \times U(1)\right) \times \operatorname{Spin}(3) \\
& \times\left\{\left(A,\left(\begin{array}{cc}
X & 0 \\
0 & x
\end{array}\right)\right) \in \mathcal{S}_{1} \times S\left(O\left(2 k_{1}-1\right) \times O(1)\right) \mid \sigma_{1}(A) x=1\right\} .
\end{aligned}
$$

Similarly, the slice representation $\sigma_{2}: K_{2} \rightarrow O(3)$ is the natural representation from the $\operatorname{Spin}(3)$-factor in $K_{2}$ to $S O(3) \subset O(3)$, and from the other factors $K_{2}^{\prime \prime}$ in $K_{2}$ to $Z S O(3)=\left\{ \pm I_{3}\right\}$. However, by $\sigma_{2}^{-1}(O(2))=K \supset K_{2}^{\prime \prime}=\psi\left(\widetilde{K_{2}^{\prime \prime}}\right)($ see $(10.1))$ and $O(2) \cap\left\{ \pm I_{3}\right\}=\left\{I_{3}\right\}$, we have that $\sigma_{2}\left(K_{2}^{\prime \prime}\right)=\left\{I_{3}\right\}$. Therefore, $K_{2} \subset G$ and $\sigma_{2}$ are completely determined by $K$.

In this case, by constructing the $G$-manifold, we can regard $\operatorname{Spin}(3)$ as $S O(3)=$ $S O\left(2 m_{b+1}+1\right)$ in $G$ up to essential isomorphism. By regarding $b+1$ as $b$ and $a-1$ as $a$, we can easily show that this case is the same as the case (3)-(b)-(i) with $k_{2}=2$ 
and $\sigma_{2}\left(K_{2}^{\prime \prime}\right)=\left\{I_{3}\right\}$. Hence, this corresponds with the second manifold in Theorem 8.1 such that $k_{2}=2$ and $\sigma_{2}: K_{2} \rightarrow S O(3) \subset O(3)$.

\section{Acknowledgement}

The author would like to thank Professor Mikiya Masuda for invaluable advices and comments on an earlier version of this paper. He also would like to thank Professor Zhi Lü for providing excellent circumstances to do research.

\section{References}

[1] G.E. Bredon, Introduction to compact transformation groups, Academic Press, 1972.

[2] V.M. Buchstaber, T.E. Panov, Torus actions and their applications in topology and combinatorics, Amer. Math. Soc., 2002.

[3] S.Y. Choi, M. Masuda, D.Y. Suh, Quasitoric manifolds over a product of simplices, to appear in Osaka J. Math; arXiv:0803.2749.

[4] M. Davis, T. Januszkiewicz, Convex polytopes, Coxeter orbifolds and torus action, Duke. Math. J., 62 (1991), no. 2, 417-451.

[5] M. Demazure, Sous-groups algebriques de rang maximum du group de Cremona, Ann. Sci. École Norm. Sup. (4) 3(1970), 507-588.

[6] W. Fulton, An Introduction to Toric Varieties, Ann. of Math. Studies 113, Princeton Univ. Press, Princeton, N.J., 1993.

[7] V. Guillemin, T.S. Holm, C. Zara, A GKM description of the equivariant cohomology ring of a homogeneous space, J. Algebraic Combin., 23 (2006), no. 1, 21-41.

[8] A. Hattori, M. Masuda, Theory of Multi-fans, Osaka. J. Math., 40 (2003), 1-68.

[9] A. Kollross, A Classification of hyperpolar and cohomogeneity one actions, Trans. Amer. Math. Soc., 354 (2002), no. 2, 571-612.

[10] S. Kuroki, On transformation groups which act on torus manifolds, Proceedings of 34th Symposium on Transformation Groups, 10-26, Wing Co., Wakayama, 2007.

[11] S. Kuroki, Classification of compact transformation groups on complex quadrics with codimension one orbits, Osaka J. Math. 46, (2009), 21-85.

[12] S. Kuroki, Characterization of homogeneous torus manifolds, to appear in Osaka J. Math.

[13] S. Kuroki, Classification of (quasi)toric manifolds with codimension one extended actions, preprint.

[14] M. Masuda, Unitary toric manifolds, Multi-fans and Equivariant index, Tôhoku Math. J., 51 (1999), 237-265.

[15] M. Mimura, H. Toda, Topology of Lie Groups, I and II, Amer. Math. Soc., 1991.

[16] P. Orlik, F. Raymond, Actions of the torus on 4-manifolds. I, Trans. Amer. Math. Soc., 152 (1970), 531-559

[17] F. Uchida, Classification of compact transformation groups on cohomology complex projective spaces with codimension one orbits, Japan. J. Math. Vol. 3, No. 1, (1977), 141-189.

[18] I. Yokota, Groups and Representations, Shokabou, 1973, (Japanese).

School of Mathematical Science Fudan University, Shanghai, 200433, P.R. China, Current Address: Dep. Mathematical Science, Kaist, 335 Gwahangno (373-1 GuseongDong), Yuseong-Gu, DAejeon 305-701 R. Korea

E-mail address: kuroki@kaist.ac.kr 